\title{
Smoothing toroidal crossing spaces
}

\author{
Simon Felten ${ }^{1}$, Matej Filip ${ }^{2}$ and Helge Ruddat ${ }^{1,3,4}$ \\ ${ }^{1}$ Johannes Gutenberg-Universität Mainz, Institut für Mathematik, Staudingerweg 9, 55128 Mainz, Germany; \\ E-mail: sfelten@uni-mainz.de. \\ ${ }^{2}$ University of Ljubljana, Institute of Mathematics, Physics and Mechanics, Trzaska cesta 25, Slovenia; \\ E-mail: matej.filip@fe.uni-lj.si. \\ ${ }^{3}$ Universität Hamburg, Fachbereich Mathematik, Bundesstraße 55, 20146 Hamburg, Germany; \\ E-mail: helge.ruddat@uni-hamburg.de. \\ ${ }^{4}$ Johannes Gutenberg-Universität Mainz, Institut für Mathematik, Staudingerweg 9, 55128 Mainz, Germany; \\ E-mail: ruddat@uni-mainz.de.
}

Received: 15 April 2021; Accepted: 18 July 2021

2020 Mathematics Subject Classification: Primary - 14J32; Secondary - 32G05, 14J45, 14D15

\begin{abstract}
We prove the existence of a smoothing for a toroidal crossing space under mild assumptions. By linking log structures with infinitesimal deformations, the result receives a very compact form for normal crossing spaces. The main approach is to study log structures that are incoherent on a subspace of codimension 2 and prove a Hodge-de Rham degeneration theorem for such log spaces that also settles a conjecture by Danilov. We show that the homotopy equivalence between Maurer-Cartan solutions and deformations combined with Batalin-Vilkovisky theory can be used to obtain smoothings. The construction of new Calabi-Yau and Fano manifolds as well as Frobenius manifold structures on moduli spaces provides potential applications.
\end{abstract}

\section{Introduction}

For two smooth components $Y_{1}, Y_{2}$ meeting in a smooth divisor $D$ a folkloristic statement says that a necessary condition for $X=Y_{1} \cup Y_{2}$ to have a smoothing is that the two normal bundles are dual to each other; that is, $\mathcal{N}_{D / Y_{1}} \otimes \mathcal{N}_{D / Y_{2}} \cong \mathcal{O}_{D}$. This statement is actually incorrect. It is true only with the further requirement that the total space of the smoothing be itself smooth. Conceptually, $\mathcal{N}_{D / Y_{1}} \otimes \mathcal{N}_{D / Y_{2}} \cong \mathcal{E} x t^{1}\left(\Omega_{X}, \mathcal{O}_{X}\right)=: \mathcal{T}_{X}^{1}$ and Friedman famously coined the notion of $d$-semistability, which is saying $\mathcal{T}_{X}^{1} \cong \mathcal{O}_{D}$ [17]. We are going to generalise the situation by only requiring $\mathcal{T}_{X}^{1}$ to be generated by global sections (and beyond). For a choice of $s \in \Gamma\left(D, \mathcal{T}_{X}^{1}\right)$, the total space of the smoothing will then be of the local form $x y=t f$ where $t$ is the deformation parameter, $Y_{1}=V(x), Y_{2}=V(y)$ and $f$ represents $s$ in a local trivialisation of $\mathcal{T}_{X}^{1}$. The total space of the smoothing has singularities precisely along $s=0$. The local form $x y=t f$ has been found to be abundant in mirror symmetry applications $[9,19,20,21,6,18,1,43]$.

We work more generally with a normal crossing space; that is, a connected variety $X$ over $\mathbb{C}$ étale locally of the form $z_{1} \cdot \ldots \cdot z_{k}=0$ for varying $k \leq \operatorname{dim} X+1$. We call a flat map $\mathcal{X} \rightarrow \mathbb{D}$ for $\mathbb{D}$ a holomorphic disk a smoothing of $X$ if the central fibre is isomorphic to $X$ and the general fibre is smooth. If a smoothing exists, then we call $X$ smoothable. We say that a normal crossing space has effective anticanonical class if the dual of its dualising sheaf $\omega_{X}$ can be represented by a reduced divisor $E$ that 
meets the strata of $X$ transversely; that is, étale locally along $E, X$ is equivalent to $E \times \mathbb{A}^{1}$. We prove the following theorem.

Theorem 1.1. Let $X$ be a proper normal crossing space with effective anticanonical class. If $\mathcal{T}_{X}^{1}$ is generated by global sections and $X_{\text {sing }}$ is projective, then $X$ is smoothable.

The only purpose of the projectivity condition is to apply Bertini's theorem to have available a

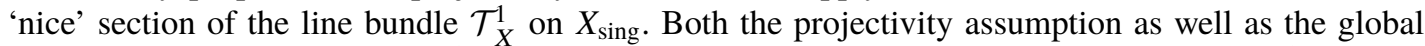
generatedness assumption on $\mathcal{T}_{X}^{1}$ can thus be removed if there exists a schön section of $\mathcal{T}_{X}^{1}$; that is, a section whose vanishing locus $Z$ is reduced and $X_{\text {sing }}$ is locally along $Z$ equivalent to $Z \times \mathbb{A}^{1}$. We also prove a more general theorem for toroidal crossing spaces (Theorem 1.7). Theorem 1.1 provides a lot more flexibility than existing smoothing results, notably Friedman's [17] for surfaces, KawamataNamikawa's [34] for d-semistable Calabi-Yaus and Gross-Siebert's [21] allowing a singular total space but with much stronger requirements on $X$; see also [51, 52, 53, 26, 36].

Example 1.2. The union $X$ of $d$ hyperplanes in general position in $\mathbb{P}^{n}$ is smoothable to a degree $d$ hypersurface, but none of the existing results is able to predict the smoothability of $X$ abstractly. Indeed, the total space of the smoothing is singular because it requires blowing up the base locus of the smoothing pencil. On the other hand, $\mathcal{T}_{X}^{1}$ is generated by global sections. Theorem 1.1 predicts the smoothability if $d \leq n+1$.

Example 1.3. The simplest type of normal crossing space is one with two smoothly intersecting components: let $Y$ be a smooth Fano manifold with $-K_{Y}$ very ample, let $D$ be a smooth section of $-K_{Y}$ and let $X$ be the normal crossing space obtained by identifying two copies of $Y$ along $D$. Then $\mathcal{T}_{X}^{1} \cong \mathcal{N}_{D / Y}^{\otimes 2}$ is generated by global sections and $X$ is Calabi-Yau, so Theorem 1.1 provides a smoothing of $X$. For Fano 3-folds $Y$ that are complete intersections in products of weighted projective spaces the smoothing gives Calabi-Yau threefolds of Euler numbers $-106,-122,-138,-156,-128,-156,-176,-256,-260,-296$. Though double intersection situations can be birationally modified to be tractable by the smoothing result in [34], this is no longer true for triple (and higher) intersection situations [35], but Theorem 1.1 provides smoothings.

Theorem 1.1 considerably facilitates the construction of Calabi-Yau and Fano manifolds. Our work generalises the Gross-Siebert program towards allowing nontoric components in the central fibre as well as more flexibility in the local structure (cf. Example 1.8). We generalise Tziolas's smoothing result for Fanos by dropping its cohomological condition [51]. Though we work with toric local models, nontoric deformations of toric local models have applications for smoothing singular toric Fanos or the construction of versal deformations of nonisolated Gorenstein singularities; see [11, 10]. For Whitney umbrella local models, the $\mathcal{T}^{1}$-sheaf has recently been computed in [14]. If the pushforward of the sheaf of differentials from the log smooth locus can be verified to commute with base change for other types of local models, then our smoothing result extends to such situations.

Our results enable the construction of versal Calabi-Yau families and conjecturally a logarithmic Frobenius manifold structure in a formal neighbourhood of the extended moduli space; see [3], [7, Theorem 1.3]. Theorem 1.10 can be viewed as the statement that the Hodge bundles extend trivially over boundary divisors in the moduli space that have toroidal families above them; see also [37]. Because the smoothing deformations are constructed via the Batalin-Vilkovisky formalism in the Gerstenhaber algebra of (log) polyvector fields (Subsection 13.1), the connection to homological mirror symmetry can be made via [3], [33].

\subsection{Method of Proof}

The first step towards proving Theorem 1.1 is to furnish $X$ with a $\log$ structure, an idea already found in $[34,21]$. We build a connection between these two works. A sheaf of sets $\mathcal{L S}_{X}$ on $X$ classifying $\log$ smooth structures locally on $X$ over the standard $\log$ point $S$ has been defined and studied in [19]. 
We show in Section 5 that there is a canonical map $\mathcal{L} \mathcal{S}_{X} \rightarrow \mathcal{T}_{X}^{1}$ with the property that a section $s \in \Gamma\left(X_{\text {sing }}, \mathcal{T}_{X}^{1}\right)$ yields a $\log$ smooth structure on $U:=X \backslash V(s)$; that is, we obtain a $\log$ smooth morphisms $U \rightarrow S$. The complement $Z:=V(s)$ has codimension 2 in $X$. Using Bertini's theorem with the projectivity of $X_{\text {sing }}$, we can assume that $Z$ is schön as defined above.

In the fashion of Zariski-Steenbrink-Danilov, we consider the differential forms $W_{X / S}^{k}:=j_{*} \Omega_{U / S}^{k}$ for $j: U \hookrightarrow X$ the inclusion. In the logarithmic context, these complexes were defined and studied independently by [38] and [20]. A key ingredient for the smoothing of $X$ is the knowledge that the Hodge-de Rham spectral sequence for $W_{X / S}^{\bullet}$ degenerates at $E_{1}$, a very hard problem. We use the close control over $W_{X / S}^{k}$ along $Z$, which we gain by using [20,41] to obtain a particular type of elementary log toroidal local model for the log structure near $Z$. For the proof of the Hodge-de Rham degeneration, we follow the approach by Deligne-Illusie [13]: spreading out to finite characteristic and using the Cartier isomorphism. However, the lack of coherence poses serious new challenges. The hardest technical part is to show that the sheaves $W_{X / S}^{\bullet}$ commute with base change because $j_{*}$ and $\otimes$ do not commute in general. Base change may fail for low characteristics by Example 7.5. However, if the characteristic of the base field is sufficiently large, we prove by explicit computation in the elementary log toroidal local models that the sheaves $W_{X / S}^{\bullet}$ commute with base change. As a second difficulty, underived pushforward $j_{*}$ does not ordinarily pass to the derived category and we find a workaround here. We settle a conjecture by Danilov [12, 14.8] along the way (Theorem 1.4).

To show the unobstructedness of $\log$ deformations of $X$, we use recent advancements of the Bogomolov-Tian-Todorov theory motivated by the study of mirror symmetry, starting with [33] and [3], which was cultivated to work in algebraic geometry by [28]. All of these works, however, produce equisingular deformations (because they are intended for deforming smooth spaces). The crucial difference to our setup is that though we prescribe local deformations by the log structure, these are not locally trivial deformations. Building on [16], recently this difficulty in the theory has been addressed in $[7,8]$, which adapts perfectly to our situation to produce a formal deformation in the prescribed local models; see Section 13. We found the framework of Gerstenhaber algebras to be the most effective to think about the theory that governs our way of parsing [7] in Subsection 13.1; see also [15]. At this point, the assumption about effectiveness of $\omega_{X}^{-1}$ enters the proof, so that one obtains an isomorphism of $W_{X / S}^{\bullet}(\log E)$ with the Gerstenhaber algebra of $\log$ polyvector fields $\mathrm{PV}^{\bullet}$ and has the Batalin-Vilkovisky operator $\Delta$ available by transporting the de Rham differential to $\mathrm{PV}^{\bullet}$, which is used in Subsection 13.2.

To improve the resulting formal smoothing to an analytic smoothing, we use the Grauert-Douady space and Artin approximation as already done in [43].

\subsection{Toroidal Pairs and Danilov's Conjecture}

A toroidal pair $(X, D)$ is a variety $X$ over a field $\mathbb{k}$ of characteristic zero with Weil divisor $D \subset X$ such that $X$ is étale locally equivalent to an affine toric variety with $D$ identified with a reduced toric divisor (not necessarily the entire toric boundary). Danilov defined the sheaf of differentials $\tilde{\Omega}_{X}^{p}(\log D)$ as the pushforward of the usual Kähler differentials $\Omega_{X_{\text {reg }}}^{p}\left(\left.\log D\right|_{X_{\text {reg }}}\right)$ with $\log$ poles from the locus $X_{\text {reg }} \subset X$ where the space is regular.

Theorem 1.4 (Danilov's conjecture). Given a proper toroidal pair $(X, D)$, the Hodge-de Rham spectral sequence

$$
E_{1}^{p, q}=H^{q}\left(X, \tilde{\Omega}_{X}^{p}(\log D)\right) \Rightarrow \mathbb{H}^{p+q}\left(X, \tilde{\Omega}_{X}^{\bullet}(\log D)\right)
$$

degenerates at $E_{1}$.

Special cases of this theorem were known before: when $X$ has at worst orbifold singularities [45], for $D=\emptyset$ [5], and for $D$ locally the entire toric boundary [48, 29]. We believe that our methods can be extended to prove generalisations of the Akizuki-Nakano-Kodaira vanishing theorem. 


\subsection{Toroidal Crossing Spaces, Their Log Structures, and Orbifold Smoothings}

If $V=\operatorname{Spec} \mathbb{k}[P]$ is an affine toric variety given by some toric monoid $P$, consider the map of sheaves $a: \underline{P} \rightarrow \mathcal{O}_{V}, p \mapsto z^{p}$, with $\underline{P}$ denoting the constant sheaf. We obtain a sheaf of monoids $\mathcal{P}_{V}=\underline{P} / a^{-1}\left(\mathcal{O}_{V}^{\times}\right)$. Now $V$ is Gorenstein if and only if the toric boundary $D$ in $V$ is a Cartier divisor and hence given as the zero locus of a monomial $\mathbb{1} \in P$.

Definition 1.5 (Schröer and Siebert [44]). A toroidal crossing space is an algebraic space $X$ over $\mathbb{k}$ together with a sheaf of monoids $\mathcal{P}$ with global section $\mathbb{1} \in \Gamma(X, \mathcal{P})$ such that for every point $x \in X$, étale locally at $x, X$ permits a smooth map to the toric boundary $D_{x}$ in $V_{x}=\operatorname{Spec} \mathbb{k}\left[\mathcal{P}_{x}\right]$ so that $\mathcal{P}$ is isomorphic to the pullback of $\mathcal{P}_{V_{x}}$ and mapping $\mathbb{1}_{x}$ to the monomial in $\mathcal{P}_{x}$ whose divisor is $D_{x}$.

A toroidal crossing space $X$ is automatically Gorenstein, and we denote its dualising line bundle by $\omega_{X}$. The boundary divisor in a Gorenstein toric variety is naturally a toroidal crossing space. General hyperplane sections of projective toroidal crossing spaces are again naturally toroidal crossing spaces.

Lemma 1.6. A normal crossing space is naturally a toroidal crossing space by setting $\mathcal{P}_{x}:=\mathbb{N}^{k}$ and $\mathbb{1}_{x}=(1,1, \ldots, 1) \in \mathbb{N}^{k}$ whenever $X$ is locally at $x$ given by $z_{1} \cdot \ldots \cdot z_{k}=0$. (Though there are other possibilities to turn a normal crossing space into a toroidal crossing space, we will always refer to this one.)

The class of toroidal crossing spaces is closed under forming products (but not so the class of normal crossing spaces). The sheaf $\mathcal{P}$ provides what Gross and Siebert call a ghost structure for $X$ ([19, Definition 3.16]), an ingredient to define the sheaf $\mathcal{L} \mathcal{S}_{X}$ ([19, Definition 3.19]) whose sections are in bijection with $\log$ structures on $X$ together with a $\log$ smooth map to the standard log point $S$. By [19], $\mathcal{L} \mathcal{S}_{X}$ embeds into the coherent sheaf $\bigoplus_{C} j_{C, *} \mathcal{N}_{\tilde{C}}$ where the sum is over the irreducible components $C$ of $X_{\text {sing }}, j_{C}: \tilde{C} \rightarrow C \rightarrow X$ is the composition of normalisation and closed embedding and $\mathcal{N}_{\tilde{C}}$ is a line bundle on $\tilde{C}$. The sheaf $\mathcal{L} \mathcal{S}_{X}$ often does not have global sections. It suffices, however, to give a section $s$ of $\mathcal{L S}_{X}$ on a dense open set $U$ that contains the generic points of the minimal strata of $X$ so that each component $s_{C} \in \Gamma\left(U \cap C, \mathcal{N}_{C}\right)$ of $s$ extends to a section of $\mathcal{N}_{C}$ on all of $C$ by acquiring simple zeros. The zeros define a reduced Cartier divisor $Z_{\tilde{C}}$ for each $\tilde{C}$. Set $Z=\cup_{C} j_{C}\left(Z_{\tilde{C}}\right) \subset X$. The construction of local models along $Z$ in [20] was generalised in [41]: locally the coherent $\log$ structure, given by $s$ on $U$, extends to an incoherent log structure on $X$ that is still given by certain toric local models, namely, from a divisor in an affine toric variety that is not the entire toric boundary; for example, as in the definition of toroidal pair above. A section $s$ of $\mathcal{L} \mathcal{S}_{X}$ on a dense open set $U$ will be called simple if it extends to $X$ by simple zeros and the resulting $Z_{\tilde{C}}$ satisfy the simpleness criterion in Section 6 . Our most general smoothing result is the following.

Theorem 1.7. Let $X$ be a proper toroidal crossing space with a simple section s of $\mathcal{L S}_{X}$ on a dense open set $U$. Assume that $\omega_{X}^{-1}$ permits a section whose divisor of zeros E meets all strata of $X$ and $Z$ transversely (e.g., when $\omega_{X}^{-1} \cong \mathcal{O}_{X}, E=\emptyset$ ); then $X$ is smoothable to an orbifold with terminal singularities.

There is a precise derivation of the types of singularities of the orbifold smoothing from knowing $\mathcal{P}$ and $Z$; for example, for a normal crossing space there will be no singularities in the general fibre of the smoothing and thus, combined with the Bertini argument and linking $\mathcal{L} \mathcal{S}_{X}$ with $\mathcal{T}_{X}^{1}$, we find that Theorem 1.7 implies Theorem 1.1; see Proposition 6.10. A section of $\mathcal{L} \mathcal{S}_{X}$ is of complete intersection type (c.i.t.) as defined in [41], roughly speaking, if the log singular set satisfies a transversality assumption. A c.i.t. section gives rise to a log toroidal morphism. Theorem 6.13 does not hold for the general c.i.t. case but we obtain the following.

Example 1.8. We follow [19]. Let $(B, \mathscr{P}, \varphi)$ be a closed oriented tropical manifold with singular locus combinatorially c.i.t.; then the associated space $X_{0}(B, \mathcal{P}, s)$ with its vanilla gluing data and $\log$ structure satisfies the assumptions of Theorem 1.7 for $E=\emptyset$ if the orbifold nearby fibre models are terminal (given by elementary simplices). Smoothings for such spaces were constructed in [21] under the stronger assumption of local rigidity; for example, the quintic 3 -fold degeneration in $\mathbb{P}^{4}$ is not locally rigid but c.i.t. 


\subsection{The Hodge-de Rham Spectral Sequence}

We refer to $[32,30,19,39]$ for basic notions of $\log$ geometry. Let $f: X \rightarrow S$ be a $\log$ toroidal family as defined in Definition 4.1. A toroidal pair $(X, D)$ yields an example by giving $X$ the divisorial $\log$ structure from $D$ and making $S$ the $\log$ trivial point. The families $X$ over the standard $\log$ point featured in Theorem 1.7 give further examples. Also, a saturated relatively $\log$ smooth morphism $f: X \rightarrow S$ in the sense of [38] is an example. The complex $W_{X / S}^{\bullet}$ (see page 3) gives rise to a spectral sequence

$$
E(X / S): E_{1}^{p q}=R^{q} f_{*} W_{X / S}^{p} \Rightarrow R^{p+q} f_{*} W_{X / S}^{\bullet}
$$

Let $Q$ be a sharp toric monoid and $\mathbb{k}$ be a field of characteristic zero. We prove the following theorems.

Theorem 1.9. Let $S=\operatorname{Spec}(Q \rightarrow \mathbb{k})$ and $f: X \rightarrow S$ be a proper log toroidal family (with respect to $\left.S \rightarrow A_{Q}\right)$. Then $E(X / S)$ degenerates at $E_{1}$.

Theorem 1.9 implies Theorem 1.4 because $W_{X / S}^{p}=\tilde{\Omega}_{X}^{p}(\log D)$ whenever $f$ comes from a toroidal pair. We conjecture the statement of Theorem 1.9 to hold also for an arbitrary coherent base $S$ over a field of characteristic zero. To prove Theorem 1.9, we adapt the proof of the degeneration in [13] as follows: because $f$ is proper, it suffices to verify

$$
\sum_{p+q=n} \operatorname{dim} R^{q} f_{*} W_{X / S}^{p}=\operatorname{dim} R^{n} f_{*} W_{X / S}^{\bullet} .
$$

In Section 9, we show that $f: X \rightarrow S$ spreads out to a log toroidal family $\phi: \mathfrak{X} \rightarrow \mathcal{S}=\operatorname{Spec}(Q \rightarrow B)$ where $\mathbb{Z} \subset B \subset \mathbb{k}$ is a subring such that $B / \mathbb{Z}$ is of finite type. Spreading out of log smooth morphisms over a $\log$ trivial base has been done before in [48, Lemma 4.11.1] and we generalise the construction to more general bases and show that the local model in the log toroidal case can be preserved. Then for suitable fields $k \supset \mathbb{F}_{p}$, with $W_{2}(k)$ denoting the ring of second Witt vectors, we obtain by base change a diagram with Cartesian squares

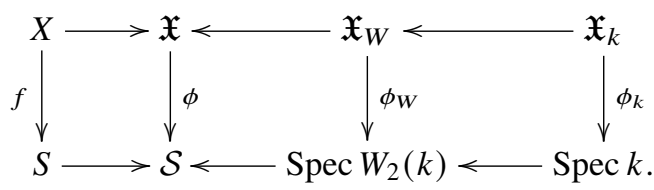

In Section 8 we investigate the behavior of $W^{\bullet}$ under base change, which leads to equalities like $\operatorname{dim}_{\mathbb{k}} R^{q} f_{*} W_{X / S}^{p}=\operatorname{dim}_{k} R^{q}\left(\phi_{k}\right)_{*} W_{\mathfrak{X}_{k} / k}^{p} ;$ that is, it suffices to show (*) for $\phi_{k}: \mathfrak{X}_{k} \rightarrow$ Spec $k$. In Section 10 we construct the Cartier isomorphism for log toroidal families in positive characteristic, which we then apply in Section 11 to obtain the Frobenius decomposition of $F_{*} W_{\mathfrak{X}_{k} / k}^{\bullet}$ where $F$ is the relative Frobenius. Finally, in Section 12, we put the pieces together and prove Theorem 1.9.

We prove a modest but important generalisation of Theorem 1.9 to the relative case using Katz's method that first appeared in [45]. This requires a detailed understanding of the analytification of the absolute differentials $W_{X}^{\bullet, a n}$ with respect to base change as given in Subsections 7.2 and 12.1.

Theorem 1.10. Let $S=S_{m}:=\operatorname{Spec}\left(\mathbb{N} \stackrel{1 \mapsto t}{\rightarrow} \mathbb{C}[t] /\left(t^{m+1}\right)\right)$ and let $f: X \rightarrow S$ be a proper log toroidal family with respect to $S \rightarrow A_{\mathbb{N}}$. Then

1. $R^{q} f_{*} W_{X / S}^{p}$ is a free $\mathbb{C}[t] /\left(t^{m+1}\right)$-module whose formation commutes with base change.

2. The spectral sequence $R^{q} f_{*} W_{X / S}^{p} \Rightarrow R^{p+q} f_{*} W_{X / S}^{\bullet}$ degenerates at $E_{1}$.

There are problems with similar theorems in earlier works: the generalisation from a 1-dimensional base to higher dimensions in [34, p. 404] is flawed, which then also affects [20, Theorem 4.1]. In addition, there is a gap in the proof of [20, Theorem 4.1] related to the fact that the de Rham differential of $\Omega_{X / S}^{\bullet}$ 
is not $\mathcal{O}_{X}$-linear. Because our result encompasses the one-parameter base case of [20, Theorem 4.1], Theorem 1.10 closes the latter gap.

Conventions. We use $\underline{X}$ to refer to the underlying scheme of a log scheme $X$. Given a map $P \rightarrow A$ from a monoid $P$ into the multiplicative monoid of a ring $A$, we refer to the associated $\log$ scheme by $\operatorname{Spec}(P \rightarrow A)$.

\section{Generically Log Smooth Families}

A $\log$ toroidal family will be a generalisation of a saturated log smooth morphism. We first introduce the weaker notion of a generically log smooth family that already enjoys some useful properties. Log structures in the entire article are assumed to be in the étale (or analytic) topology. If $f: X \rightarrow S$ is a finite-type morphism of Noetherian schemes, we say a Zariski open $U \subset X$ satisfies the codimension condition (CC) if the relative codimension of $Z:=X \backslash U$ is $\geq 2$; that is, for every point $s \in S$ with $X_{s}, U_{s}$ the fibres,

$$
\operatorname{codim}\left(X_{s} \backslash U_{s}, X_{s}\right) \geq 2
$$

A Cohen-Macaulay morphism is a flat morphism with Cohen-Macaulay fibres.

Definition 2.1. A generically log smooth family consists of

$\circ$ a finite-type Cohen-Macaulay morphism $f: X \rightarrow S$ of Noetherian schemes,

- a Zariski open $j: U \subset X$ satisfying (CC),

- a saturated and $\log$ smooth morphism $f:\left(U, \mathcal{M}_{U}\right) \rightarrow\left(S, \mathcal{M}_{S}\right)$ of fine saturated log schemes.

The complement $Z:=X \backslash U$ we refer to as the $\log$ singular locus even though $f$ might extend $\log$ smoothly to it. We say two generically $\log$ smooth families $f, f^{\prime}: X \rightarrow S$ with the same underlying morphism of schemes are equivalent, if there is some $\tilde{U} \subset U \cap U^{\prime}$ satisfying $(C C)$ with $\left.\left.\mathcal{M}_{U}\right|_{\tilde{U}} \cong \mathcal{M}_{U^{\prime}}^{\prime}\right|_{\tilde{U}}$ compatibly with all data.

If $T \rightarrow S$ is a morphism of fine saturated log schemes, then the base change $X_{T} \rightarrow T$ as a generically $\log$ smooth family is defined in the obvious way, taking fibre products in the category of all $\log$ schemes. Note that we need $f: U \rightarrow S$ saturated to ensure that $U_{T}$ is again a fine saturated log scheme. The notion of equivalence is due to the fact that we do not care about the precise $U$. However, for technical simplicity we assume some $U$ fixed. The name log singular locus is in analogy with [19].

Definition 2.2. For a generically $\log$ smooth family $f: X \rightarrow S$, the de Rham complex is defined as $W_{X / S}^{\bullet}:=j_{*} \Omega_{U / S}^{\bullet}$ where $\Omega_{U / S}^{\bullet}$ denotes the log de Rham complex. We also define the $\mathcal{O}_{X}$-module of degree $m \log$ polyvector fields $\Theta_{X / S}^{m}:=j_{*} \bigwedge^{m} \operatorname{Der}_{U / S}\left(\mathcal{O}_{U}\right)$.

Lemma 2.3. Let $f: X \rightarrow S$ be a Cohen-Macaulay morphism of Noetherian schemes, and let $j: U \subset X$ satisfy $(C C)$. Then $j_{*} \mathcal{O}_{U} \cong \mathcal{O}_{X}$.

Proof. This is a special case of [27, Proposition 3.5]. Note that our (CC) is a stronger assumption than the condition on the codimension in [27, Proposition 3.5].

Let $X \rightarrow S$ be a generically log smooth family. Using the language of [24, Definition 5.9.9], a sheaf $\mathcal{F}$ we call $Z$-closed if the natural map $\mathcal{F} \rightarrow j_{*}\left(\left.\mathcal{F}\right|_{U}\right)$ is an isomorphism. Most notable, two Zclosed sheaves that agree on $U$ are entirely equal. By their definition, $W_{X / S}^{m}$ and $\Theta_{X / S}^{m}$ are $Z$-closed. Furthermore, every reflexive sheaf is $Z$-closed.

Lemma 2.4. The $\mathcal{O}_{X}$-modules $W_{X / S}^{m}$ and $\Theta_{X / S}^{m}$ are coherent and reflexive and these depend only on the equivalence class of $f: X \rightarrow S$. 
Proof. Let $\tilde{U} \subset U$ also satisfy $(C C)$. We have by Lemma 2.3 that $j_{*} \Omega_{\tilde{U} / S}^{\bullet}=j_{*} \Omega_{U / S}^{\bullet}$ because $\Omega_{U / S}^{m}$ is finite locally free. Thus, $W_{X / S}^{\bullet}$ depends only on the equivalence class of $f$. It is clear that it is quasi-coherent. For every sheaf $\mathcal{G}$ on $U, j_{*} \mathcal{G}$ is $Z$-closed, so in particular $W_{X / S}^{m}$ is $Z$-closed. Set $\mathcal{F}^{\vee}:=$ $\operatorname{Hom}_{\mathcal{O}_{X}}\left(\mathcal{F}, \mathcal{O}_{X}\right)$. By Lemma 2.3, $\mathcal{F}^{\vee}$ is $Z$-closed for all $\mathcal{F}$, so in particular $\left(W_{X / S}^{m}\right)^{\vee \vee}$ is a $Z$-closed sheaf and it coincides with $W_{X / S}^{m}$ on $U$; hence, $\left(W_{X / S}^{m}\right)^{\vee \vee}=W_{X / S}^{m}$ and $W_{X / S}^{m}$ is reflexive. By the extension theorem [22, Corollaire 9.4.8], there is a coherent $\mathcal{G}$ that restricts to $W_{X / S}^{m}$ on $U$. Now $\mathcal{G}^{\vee \vee}=W_{X / S}^{m}$ because both are $Z$-closed and agree on $U$; hence, $W_{X / S}^{m}$ is also coherent. The argument for $\Theta_{X / S}^{m}$ is similar.

Lemma 2.5. $W_{X / S}^{m}=\mathcal{H o m}\left(\Theta_{X / S}^{m}, \mathcal{O}_{X}\right)$ and $\Theta_{X / S}^{m}=\mathcal{H o m}\left(W_{X / S}^{m}, \mathcal{O}_{X}\right)$.

Proof. The statement is clear on $U$ where all sheaves are locally free and then it follows because all sheaves are $Z$-closed.

Remark 2.6. The pushforward $j_{*} \mathcal{M}_{U} \rightarrow j_{*} \mathcal{O}_{U}=\mathcal{O}_{X}$ to $X$ yields a log structure that is compatible with $S$, so every generically $\log$ smooth family is canonically a $\log$ morphism $X \rightarrow S$. We do not know whether this pushforward is compatible with base change (and we do not care).

Remark 2.7. In view of Remark 2.6, neither the so-defined log structure $\mathcal{M}_{X}$ nor the associated sheaf of $\log$ differentials $\Omega_{X / S}$ will be coherent in general; see Example 2.11. On the the other hand, $W_{X / S}^{m}$ and $\Theta_{X / S}^{m}$ are coherent and have further good properties in the case of log toroidal families, as we will see.

Let $X \rightarrow S$ be a generically $\log$ smooth family. One defines for the $\log$ smooth morphism $U \rightarrow S$ the horizontal divisor $D_{U} \subset U$ (see, e.g., [49, Definition 2.4] and Remark 3.2). This is only a Weil divisor in general. We denote by $D$ its closure in $X$ and by $I_{D}$ the corresponding ideal sheaf. We define $W_{X / S}^{m}(-D):=j_{*}\left(\left.\left(I_{D} W_{X / S}^{m}\right)\right|_{U}\right)$. (This does not need to agree with $I_{D} W_{X / S}^{m}$.)

Proposition 2.8. Let $S=\operatorname{Spec}(\mathbb{N} \rightarrow \mathbb{k})$ for $\mathbb{k}$ a field where $1 \mapsto 0$. Let $f: X \rightarrow S$ be a generically log smooth family of relative dimension d and let $\omega_{f}=f^{!} \mathcal{O}_{S}$ denote the (globally normalised) relative dualising sheaf; then

$$
W_{X / S}^{d}(-D)=\omega_{f}
$$

Proof. On $U$, this is [49, Theorem 2.21, (ii)], and because both sheaves are Z-closed, the statement follows.

Example 2.9. Let $f: X \rightarrow S$ be a $\log$ smooth and saturated morphism of Noetherian fine saturated $\log$ schemes. Then $f$ is flat by [32, Corollaire 4.5] and has Cohen-Macaulay fibres by [50, Proposition II.4.1]. We see that $f: X \rightarrow S$ gives a generically $\log$ smooth family for $U=X$ and $W_{X / S}^{\bullet}$ is the ordinary log de Rham complex.

Not every log smooth morphism is saturated; for example, see [30, Remark 9.1] for a log smooth morphism that is not even integral.

Example 2.10. Let $X / \operatorname{Spec} R$ be a toric variety over a Noetherian base ring $R$. The fibres over points in Spec $R$ are normal (and Cohen-Macaulay), so there is a regular open $U \subset X$ whose complement has relative codimension $\geq 2$ over $\operatorname{Spec} R$. For every divisorial log structure on $X$ coming from a torusinvariant divisor $D$ on $X$, the map $U \rightarrow$ Spec $R$ is $\log$ smooth and saturated when using the trivial $\log$ structure on Spec $R$. Hence, $X \rightarrow \operatorname{Spec} R$ is a generically $\log$ smooth family. The differentials $W_{X / S}^{\bullet}$ coincide with what is called reflexive or Danilov or Zariski-Steenbrink differentials with log poles in $D$. This example extends to toroidal pairs $(X, D)$ over $\operatorname{Spec} R$.

Example 2.11. The $\mathbb{Z}[t]$-algebra $A=\mathbb{Z}[x, y, t, w] /(x y-t w)$ defines a map $f: \operatorname{Spec} A \rightarrow \mathbb{A}^{1}$ that is $\log$ smooth and saturated away from the origin when using the divisorial log structure given by $t=0$ on source and target and hence a generically $\log$ smooth family. The $\log$ structure on $\operatorname{Spec} A$ is not 
coherent at the origin, so $f$ is not $\log$ smooth. Even worse, $\Omega_{f}$ is not a coherent sheaf at the origin; see [20, Example 1.11].

Another type of generically log smooth family with application to Gromov-Witten theory can be found in [4].

\subsection{Analytification}

Given a generically $\log$ smooth family $f: X \rightarrow S$ of finite type over $\mathbb{C}$, we denote the associated family of complex analytic spaces by $f^{a n}: X^{a n} \rightarrow S^{a n}$. Induced by $f$, the open $U^{a n} \subset X^{a n}$ carries an fs $\log$ structure so that $U^{a n} \rightarrow S^{a n}$ is a $\log$ smooth and saturated morphism of fs $\log$ analytic spaces. The analogue of Lemma 2.3 holds if $X^{a n}, S^{a n}$ are Cohen-Macaulay by [2, Theorem 3.6]. For $S=\operatorname{Spec}(Q \rightarrow A)$ with $A$ an Artinian ring and

$$
W_{X / S}^{\bullet, a n}:=j_{*}^{a n} \Omega_{U^{a n} / S^{a n}}^{\bullet}
$$

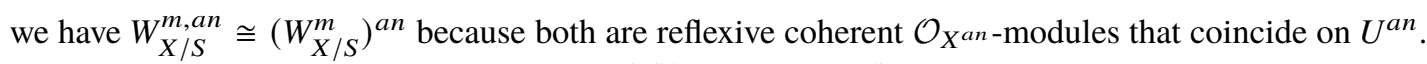
If $f$ is proper, then GAGA gives $H^{q}\left(X^{a n}, W_{X / S}^{p, a n}\right) \cong H^{q}\left(X, W_{X / S}^{p}\right)$ and also

$$
\mathbb{H}^{p+q}\left(X^{a n}, W_{X / S}^{\bullet, a n}\right) \cong \mathbb{H}^{p+q}\left(X, W_{X / S}^{\bullet}\right)
$$

for example, via the comparison of the Hodge-de Rham spectral sequences.

\section{Elementary Log Toroidal Families}

For basic notions and constructions of monoids, see [39].

Definition 3.1. An elementary $(\log )$ toroidal datum $(Q \subset P, \mathcal{F})$ (ETD for short) consists of an injection $Q \hookrightarrow P$ of sharp toric monoids that turns $P$ into a free $Q$-set whose canonical basis is a union of faces of $P$. We furthermore record a set $\mathcal{F}$ of facets of $P$ with the property that it contains all facets that do not contain $Q$. In other words, if we define

$$
\mathcal{F}_{\text {min }}:=\underbrace{\{F \subset P \text { a facet } \mid Q \not \subset F\}}_{\text {vertical facets }},
$$

then $\mathcal{F}_{\text {min }} \subset \mathcal{F} \subset \mathcal{F}_{\text {max }}$ where $\mathcal{F}_{\text {max }}$ is the set of all facets.

Remark 3.2. The facets in $\mathcal{F} \backslash \mathcal{F}_{\text {min }}$ will give the horizontal divisor that we referred to as $D$ before.

Lemma 3.3. ([39, Corollary I.4.6.11, Theorem I.4.8.14, Corollary I.1.4.3]). The requirement on the injection $Q \hookrightarrow P$ in Definition 3.1 is equivalent to saying that this map is saturated.

See Figure 3.1 for examples. Even the case $Q=0$ can be interesting because then $\mathcal{F}_{\min }=\emptyset$. We denote the union of faces of $P$ that gives the generating set of the free $Q$-action by $E$. A face $F$ of $P$ contained in $E$ we call an essential face. Every $p \in P$ has a unique decomposition $p=e+q$ with $e \in E, q \in Q$; hence,

$$
E \times Q \rightarrow P, \quad(e, q) \mapsto e+q,
$$

is bijective ([39, Theorem I.4.8.14]; cf. [31, Lemma 1.1]). Furthermore, we see that $E=P \backslash\left(Q^{+}+P\right)$ where $Q^{+}=Q \backslash 0$ is the maximal ideal. Moreover, projecting $E$ to $P^{\mathrm{gp}} / Q^{\mathrm{gp}}$ is injective and the set of essential faces gives a fan in $P^{\mathrm{gp}} / Q^{\mathrm{gp}}$ whose support $\bar{P}$ is convex in $\left(P^{\mathrm{gp}} / Q^{\mathrm{gp}}\right) \otimes_{\mathbb{Z}} \mathbb{R}$ because it is the convex hull of the projection of $P$. Note that $\bar{P}^{\mathrm{gp}}=P^{\mathrm{gp}} / Q^{\mathrm{gp}}$. A choice of splitting $P^{\mathrm{gp}} \cong \bar{P}^{\mathrm{gp}} \oplus Q^{\mathrm{gp}}$ 

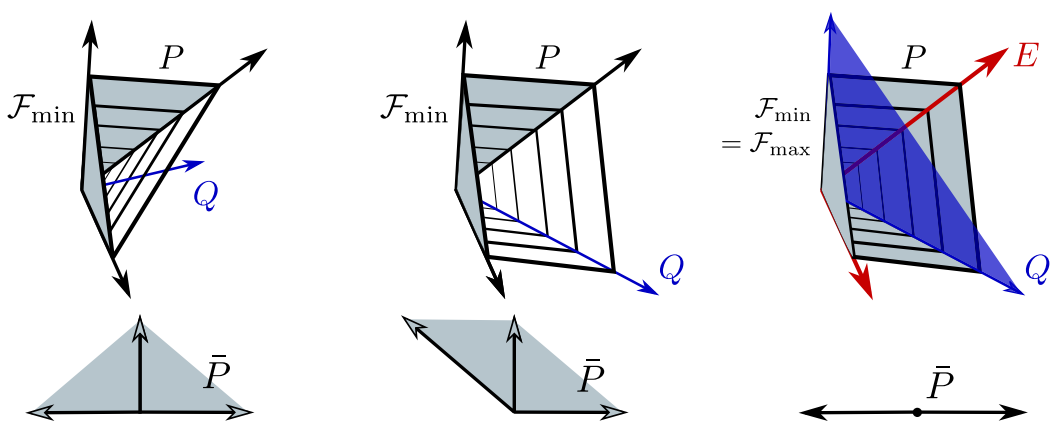

Figure 3.1. Three examples of a saturated injection $Q \subset P$ and the projection $\bar{P}$; the outer two are $\log$ smooth and the middle one gives Example 2.11.

yields a unique map of sets $\varphi: \bar{P} \rightarrow Q^{\text {gp }}$ so that $\mathrm{id} \times \varphi: \bar{P} \rightarrow \bar{P} \oplus Q^{\text {gp }}$ is a section of the projection $P \rightarrow \bar{P}$ with the property that its image is $E$, so

$$
P=\left\{(\bar{p}, q) \in \bar{P} \oplus Q^{\mathrm{gp}} \mid \exists \tilde{q} \in Q: q=\varphi(\bar{p})+\tilde{q}\right\} .
$$

Lemma 3.4. The morphism $f: \operatorname{Spec} \mathbb{Z}[P] \rightarrow \operatorname{Spec} \mathbb{Z}[Q]$ induced by the injection $Q \subset P$ is a CohenMacaulay morphism of fibre dimension $d=\operatorname{rk}\left(P^{\mathrm{gp}} / Q^{\mathrm{gp}}\right)$.

Proof. Because $P$ is free as a $Q$-set (generated by $E$ ), Spec $\mathbb{Z}[P]$ is a flat $\operatorname{Spec} \mathbb{Z}[Q]$-module. By $[24$, Corollary 6.3.5] the total space of a faithfully flat morphism of Noetherian schemes is Cohen-Macaulay if and only if the base and all fibres are. By Hoechster's theorem, the fibres of $\operatorname{Spec} \mathbb{Z}[P] \rightarrow \operatorname{Spec} \mathbb{Z}$ are Cohen-Macaulay; hence, $\operatorname{Spec} \mathbb{Z}[P]$ and $\operatorname{Spec} \mathbb{Z}[Q]$ are Cohen-Macaulay. Now flatness of $\underline{f}$ implies that it is Cohen-Macaulay.

We next want to define an open set $U$ in the domain of $f$ that satisfies (CC). We will actually define its complement and for this we need a good understanding of the faces of $P$.

Lemma 3.5. Let $F \subset P$ be a face. Set $\bar{F}:=F \cap E, Q^{\prime}:=Q \cap F$; then

$$
F=\bar{F}+Q^{\prime}:=\left\{\bar{f}+q^{\prime} \mid \bar{f} \in \bar{F}, q^{\prime} \in Q^{\prime}\right\} .
$$

Because $E$ is a union of faces of $P$, so is $\bar{F}$. Note also that $Q^{\prime}$ is a face of $Q$.

Proof. By the decomposition (3.1), any element in $F$ has the form $\bar{f}+q$ with $\bar{f} \in E, q \in Q$. Because $F$ is a face, $\bar{f}, q$ are both in $F$; hence, $F \subset \bar{F}+Q^{\prime}$. The reverse inclusion is clear.

Consider the set of bad faces of $P$ defined as

$$
\mathcal{B}=\left\{\begin{array}{c|c}
\bar{F}+Q^{\prime} & \begin{array}{c}
\bar{F} \text { is a union of essential faces of rank at most } d-2 \\
Q^{\prime} \text { is a face of } Q, \bar{F}+Q^{\prime} \text { is a face of } P
\end{array}
\end{array}\right\} .
$$

Recall that there is a one-to-one correspondence between faces $F$ of $P$ and torus orbits closures $V_{F}:=\operatorname{Spec} \mathbb{Z}[F]$ in $\operatorname{Spec} \mathbb{Z}[P]$. Similarly, for $Q^{\prime}$ a face of $Q$, we have a torus orbit closure $V_{Q^{\prime}}:=$ $\operatorname{Spec} \mathbb{Z}\left[Q^{\prime}\right] \subset \operatorname{Spec} \mathbb{Z}[Q]$.

Lemma 3.6. Given $\bar{F}+Q^{\prime} \in \mathcal{B}$, we find that $V_{\bar{F}+Q^{\prime}}$ is flat over $V_{Q^{\prime}} \subset \operatorname{Spec} \mathbb{Z}[Q]$. Furthermore, if $X$ is a fibre of $\underline{f}$, then $\operatorname{codim}\left(X \cap V_{\bar{F}+Q^{\prime}}, X\right) \geq 2$.

Proof. Because $\bar{F}+Q^{\prime}$ is free as a $Q^{\prime}$-set, $\mathbb{Z}\left[\bar{F}+Q^{\prime}\right]$ is a free $\mathbb{Z}\left[Q^{\prime}\right]$-module, so the flatness statement follows. The origin 0 given by the prime ideal $\left(z^{q} \mid q \in Q^{+}\right)$is contained in $V_{Q^{\prime}}$ and let $X_{0}$ be the fibre over it. It suffices to check the codimension condition for this particular fibre. But note that 
$X_{0} \cap V_{\bar{F}+Q^{\prime}}=\bigcup_{F \subset \bar{F}} V_{F}$ where the union runs over faces $F$ of $P$ contained in $\bar{F}$ and we have $\operatorname{dim} V_{F} \leq d-2$ by the assumption on $\bar{F}$.

Set

$$
U_{P}:=\operatorname{Spec} \mathbb{Z}[P] \backslash\left(\bigcup_{B \in \mathcal{B}} V_{B}\right) .
$$

For every face $F$ of $P$, we have an open subset $\operatorname{Spec} \mathbb{Z}\left[P_{F}\right]$ of Spec $\mathbb{Z}[P]$ where $P_{F}$ is the localisation of $P$ in $F$; that is, $P_{F}$ is the submonoid of $P^{\text {gp }}$ generated by $P$ and $-F$.

Lemma 3.7. We find $U_{P}=\bigcup_{F} U_{F}$ where the union is over the essential faces $F$ of rank $d-1$.

Proof. Because $U_{P}$ is a union of torus orbits, it suffices to check that any torus orbit contained in $U_{P}$ is contained in some $U_{F}$ for $F$ essential of rank $d-1$. Every torus orbit is given by $O_{G}:=\operatorname{Spec} \mathbb{Z}\left[G^{\mathrm{gp}}\right]$ for $G$ a face of $P$. Assume $O_{G} \subset U$. We use Lemma 3.5 to write $G=\bar{G}+Q^{\prime}$. If $\operatorname{rk} \bar{G} \leq d-2$, then $G \in \mathcal{B}$, so $O_{G} \not \subset U$. Hence, $\operatorname{dim} \bar{G} \geq d-1$ and $\bar{G}$ contains some essential face $F$ of rank $d-1$. Then $F$ is also contained in $G$ and thus $O_{G}$ is contained in $U_{F}$. Conversely, because $O_{F}$ is not in any $V_{B}$, the assertion follows.

Let $A_{Q}:=\operatorname{Spec}(Q \rightarrow \mathbb{Z}[Q])$ denote the log scheme with standard toric log structure and let $A_{P, \mathcal{F}}$ be the log scheme with underlying scheme Spec $\mathbb{Z}[Q]$ and divisorial log structure given by the divisor $\bigcup_{F \in \mathcal{F}} \operatorname{Spec} \mathbb{Z}[F]$. The map $f: A_{P, \mathcal{F}} \rightarrow A_{Q}$ induced by $\theta$ is naturally a log morphism by the condition on $\mathcal{F}$ to contain the vertical faces. We work here with Zariski log structures that coincide with the pushforward of the corresponding étale log structures by [39, Proposition III.1.6.5].

Lemma 3.8 (Theorem 3.5 in [32] or Theorem 4.1 in [30]). If $\mathcal{F}=\mathcal{F}_{\max }$, then $f$ is log smooth.

Proposition 3.9. The map $f: A_{P, \mathcal{F}} \rightarrow A_{Q}$ is a generically log smooth family with $U_{P}$ serving as the specified dense open of log smoothness.

Proof. If $\mathcal{F}=\mathcal{F}_{\text {max }}$, then $f$ is saturated because $\theta$ is saturated. More generally, because $A_{P, \mathcal{F}_{\max }} \rightarrow A_{P, \mathcal{F}}$ is locally given by embedding a face, it is exact. Now by [39, I.4.8.5(2)], $f$ is saturated.

The assertion is clear if $d=0 \Longleftrightarrow P=Q$, so assume $d>0$. Given Lemma 3.4, we still need to verify that $U$ satisfies (CC) and that $f$ is $\log$ smooth on $U_{P}$. Note that Lemma 3.6 implies that $U_{P}$ satisfies (CC) because the complement of $U_{P}$ is the union of closed sets each of which has codimension at least 2 in each fibre.

To see that $f$ is $\log$ smooth on $U_{P}$, by Lemma 3.7, it suffices to check that $f$ is log smooth on $U_{F}$ for $F$ essential of rank $d-1$. Let $F$ be such a face. Set $\bar{P}_{F}:=P_{F} / F^{\text {gp }}$ and note that the projection of $Q$ to $\bar{P}_{F}$ is injective because $F^{\mathrm{gp}} \cap Q=\{0\}$. There is an isomorphism $P_{F} \cong F^{\mathrm{gp}} \times \bar{P}_{F}$ commuting with the injection of $Q$ that is $\{0\} \times Q$ on the right.

The $\log$ structure on $U_{F}$ is a divisorial $\log$ structure given by a set of divisors each of which pulls back from Spec $\mathbb{Z}\left[\bar{P}_{F}\right]$, so we may consider the corresponding divisorial log structure on Spec $\mathbb{Z}\left[\bar{P}_{F}\right]$ to upgrade this to a log scheme $\bar{U}_{F}$. We have a factorisation $U_{F} \rightarrow \bar{U}_{F} \rightarrow A_{Q}$ with the first map a smooth projection from a product that is therefore strict and hence $\log$ smooth. It thus suffices to show that $\bar{U}_{F} \rightarrow A_{Q}$ is $\log$ smooth. Note that $\bar{U}_{F} \rightarrow A_{Q}$ is the $\log$ morphism of an ETD with $d=1$. The following lemma finishes the proof.

Lemma 3.10. Assume that $f: A_{P, \mathcal{F}} \rightarrow A_{Q}$ has 1-dimensional fibres (i.e., $d=1$ ); then $f$ is $\log$ smooth. (The third situation of Figure 3.1 is an example.)

Proof. We are done by Lemma 3.8 if $\mathcal{F}=\mathcal{F}_{\max }$, and this always holds if $Q$ meets the interior of $P$. So assume that $Q$ is contained in a proper face of $P$; then by Lemma 3.5 it is in fact a facet of $P$ and then $\bar{P}=\mathbb{N}$ and consequently $P=\mathbb{N} \times Q$. A facet of $P$ that is not $Q$ is in $\mathcal{F}_{\text {min }}=\{\mathbb{N} \times F \mid F$ is a facet of Q $\}$. Hence, $\mathcal{F} \subsetneq \mathcal{F}_{\text {max }}$ implies $\mathcal{F}=\mathcal{F}_{\text {min }}$ and thus $f$ is strict. Because $f$ is smooth, we find that $f$ is $\log$ smooth. 
Corollary 3.11. It is possible to find open subsets $U_{1}$ and $U_{2}$ so that $U_{P}=U_{1} \cup U_{2}$ and $\left.A_{P}\right|_{U_{1}}=\left.A_{P, \mathcal{F}}\right|_{U_{1}}$ and $f: U_{2} \subset A_{P, \mathcal{F}} \rightarrow A_{Q}$ is strict and smooth.

Proof. Let $\mathcal{E}_{1}$ be the set of essential faces of rank $d-1$ such that when applying the proof of Lemma 3.10 to $\bar{U}_{F} \rightarrow A_{Q}$ from the proof of the proposition, we are in the case $\mathcal{F}=\mathcal{F}_{\text {max }}$, and let $\mathcal{E}_{2}$ be the set of faces where we are in case $\mathcal{F}=\mathcal{F}_{\text {min }}$. Then for $F \in \mathcal{E}_{1}$ we have $\left.A_{P}\right|_{U_{F}}=\left.A_{P, \mathcal{F}}\right|_{U_{F}}$, and for $F \in \mathcal{E}_{2}$ the morphism $U_{F} \rightarrow A_{Q}$ is strict and smooth. Now we define $U_{1}=\bigcup_{F \in \mathcal{E}_{1}} U_{F}$ and $U_{2}=\bigcup_{F \in \mathcal{E}_{2}} U_{F}$.

Example 3.12. If $(Q \subset P, \mathcal{F})$ is an ETD and $r \geq 0$, then we obtain another ETD $\left(Q \times\{0\} \subset P \times \mathbb{N}^{r}, \mathcal{F}^{\prime}\right)$ where $\mathcal{F}^{\prime}=\left\{F \times \mathbb{N}^{r} \mid F \in \mathcal{F}\right\}$.

\section{Log Toroidal Families}

We define log toroidal families and investigate their basic properties.

Definition 4.1. We say that a generically $\log$ smooth family $f: X \rightarrow S$ is $\log$ toroidal if for every geometric point $\bar{x} \rightarrow X$, we have a commutative diagram

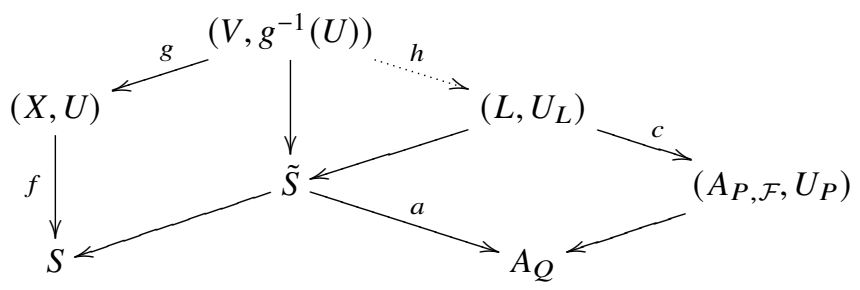

where $g: V \rightarrow X$ is an étale neighbourhood of $\bar{x}, \tilde{S} \rightarrow S$ is a strict étale neighbourhood of $f(\bar{x})$ and $a$ is given by a chart $Q \rightarrow \mathcal{M}_{\tilde{S}}$ of $\tilde{S}$. The bottom right diagonal map is required to be given by an ETD $(Q \subset P, \mathcal{F})$ and $U_{P} \subset A_{P, \mathcal{F}}$ denotes the open set from (3.3). The solid arrows are morphisms of schemes and $\log$ morphisms on the specified opens, whereas $h: V \rightarrow L$ is an étale morphism only of underlying schemes. The bottom right diamond is Cartesian, in particular, $U_{L}=c^{-1}\left(U_{P}\right)$. Moreover, we have an open $\tilde{U} \subset V$ satisfying (CC), such that $\tilde{U} \subset g^{-1}(U) \cap h^{-1}\left(U_{L}\right)$ and there is an isomorphism $g^{*} \mathcal{M}_{X} \cong h^{*} \mathcal{M}_{L}$ of the two $\log$ structures on $\tilde{U}$ compatible with the maps to $S$.

The diagram (LM) is called a local model for $f: X \rightarrow S$ at $\bar{x}$. If $S \cong \operatorname{Spec}(Q \rightarrow B)$, every point has a local model with $\tilde{S}=S$ and $a$ is given by the chart $Q \rightarrow B$, then we say that $f: X \rightarrow S$ is $\log$ toroidal with respect to $a: S \rightarrow A_{Q}$.

Log toroidal families are stable under strict base change.

Remark 4.2. Note that Definition 4.1 only requires a covering of $X$ by (LM) but does not say that an arbitrary geometric point $\bar{x} \in X$ permits a diagram (LM) that identifies $\bar{x}$ with the origin in $A_{P}$. However, if $\mathbb{k}$ is algebraically closed, one can show that by localising the ETD in (LM) and using Example 3.12, one can assume that $\bar{x} \in X$ becomes the origin in $A_{P}$. We will make use of this fact in the proof of Theorem 1.10.

Example 4.3. Every elementary $\log$ toroidal family $f: A_{P, \mathcal{F}} \rightarrow A_{Q}$ is $\log$ toroidal.

Example 4.4. The generically log smooth families given in Example 2.10 are log toroidal families with $Q=0$ in every ETD.

Example 4.5. A saturated $\log$ smooth morphism $f: X \rightarrow S$ is $\log$ toroidal with $U=X$. Indeed, locally starting from a neat chart of $f$, set $\mathcal{F}=\mathcal{F}_{\text {max }}$ and then apply Example 3.12 to have local models. That this works is not a trivial consequence of Theorem 3.5 in [32]. Instead, use [39, Theorem VI.3.3.3].

Example 4.6. In the setting and notation of the Gross-Siebert program, [20, Theorem 2.6] shows that if $(B, \mathcal{P})$ is positive and simple, and $s$ is lifted open gluing data, then $X_{0}^{\dagger}(B, \mathcal{P}, s) \rightarrow \operatorname{Spec}(\mathbb{N} \rightarrow k)$ is $\log$ 
toroidal. More generally, it was shown in [41, Proposition 2.8] that c.i.t. log Calabi-Yau spaces are log toroidal over $\operatorname{Spec}(\mathbb{N} \rightarrow k)$. The divisorial deformations defined in [20] are also log toroidal families.

\section{Log Structures and Infinitesimal Deformations}

Let $X$ be a toroidal crossing space over a field $\mathbb{k}$. As mentioned in the Introduction, $X$ can be equipped with a sheaf of sets $\mathcal{L} \mathcal{S}_{X}$, which we recall next. An alternative categorical approach to study log smooth morphisms for a fixed underlying morphism has been developed in [40], though we follow the sheaf approach. Let $S=\operatorname{Spec}(\mathbb{N} \stackrel{1 \mapsto 0}{\rightarrow} \mathbb{k})$ be the standard log point. The pair $(\mathcal{P}, \mathbb{1})$ gives a ghost structure in the sense of [19, Definition 3.16]. Indeed, the type of the ghost structure is fixed by requiring it to be the one given by the local chart that comes with the definition of a toroidal crossing space. We will refer to this type as the given type below. By [19, Definition 3.19 and Proposition 3.20], there is a sheaf $\mathcal{L S}_{X}$ (denoted $\mathcal{L S}_{X^{g}}$ in [19]) with the property that for every étale open $U \subset X$, there is a natural bijection

$$
\Gamma\left(U, \mathcal{L} \mathcal{S}_{X}\right)=\left\{\begin{array}{c|c}
\mathcal{M}_{U} \rightarrow \mathcal{O}_{U} \text { a log structure of } & \left(U, \mathcal{M}_{U}\right) \rightarrow S \text { via } 1 \mapsto \tilde{\mathbb{1}} \text { is a } \\
\text { the given type, } \tilde{\mathbb{1}} \in \Gamma\left(U, \mathcal{M}_{U}\right), & \log \text { smooth morphism and } \\
\overline{\mathcal{M}}_{U} \stackrel{\sim}{\rightarrow} \mathcal{P} \text { an isomorphism } & \mathcal{M}_{U} \rightarrow \overline{\mathcal{M}}_{U} \rightarrow \mathcal{P} \text { sends } \tilde{\mathbb{1}} \text { to } \mathbb{1}
\end{array}\right\}
$$

where the set on the right is to be taken modulo isomorphisms. The support of $\mathcal{P} / \mathbb{1}$ agrees with $X_{\text {sing, }}$, so the sheaf $\mathcal{L S}_{X}$ is supported on $X_{\text {sing }}$.

Set $S_{\varepsilon}:=\operatorname{Spec}\left(\mathbb{N} \stackrel{1 \mapsto \varepsilon}{\rightarrow} \mathbb{k}[\varepsilon] /\left(\varepsilon^{2}\right)\right)$. If $V \rightarrow S$ is a log smooth morphism with $V$ affine, then there is a unique $\log$ smooth lifting $V_{\varepsilon} \rightarrow S_{\varepsilon}$ up to isomorphism. For $(\mathcal{M}, \tilde{\mathbb{1}}) \in \mathcal{L S}_{X}(U)$ and an affine $V \subset U$, the deformation $i: V \rightarrow V_{\varepsilon}$ yields an extension

$$
0 \rightarrow \mathcal{O}_{V} \rightarrow i^{*} \Omega_{\underline{V_{\varepsilon}}}^{1} \rightarrow \Omega_{\underline{V}}^{1} \rightarrow 0
$$

where on the left $1 \mapsto i^{*} d \varepsilon$. The classes of such local extensions glue to a well-defined class in $\mathcal{E} x t^{1}\left(\Omega_{U}^{1}, \mathcal{O}_{U}\right)$ (though neither the extensions nor the deformations need to glue). We have thus defined a map of sheaves of sets

$$
\eta: \mathcal{L} \mathcal{S}_{X} \rightarrow \mathcal{E} x t^{1}\left(\Omega_{X}^{1}, \mathcal{O}_{X}\right)=\mathcal{T}_{X}^{1}
$$

Remark 5.1. In this form, the map $\eta$ seems to be new. However, a close relationship between $\log$ structures and $\mathcal{T}_{X}^{1}$ has been observed before in [34, Proposition 1.1], [46, Remark (3.11)], [30, Theorem 11.7], [19, Example 3.30], [40, Theorem 3.18], [44, Theorem 7.5].

Both sheaves in (5.2) have a natural action of $\mathcal{O}_{X}^{\times}$: indeed, $\mathcal{T}_{X}^{1}$ because it is coherent and for $\mathcal{L} \mathcal{S}_{X}$ we let a section $\lambda$ of $\mathcal{O}_{X}^{\times}$act by $\tilde{\mathbb{1}} \mapsto \lambda^{-1} \tilde{\mathbb{1}}$.

Proposition 5.2. The map $\eta$ is $\mathcal{O}_{X}^{\times}$-equivariant.

Proof. At a geometric point $\bar{x} \in X$ with $M=(\mathcal{M}, \tilde{\mathbb{1}}) \in \mathcal{L} \mathcal{S}_{X, \bar{x}}$ for $\mathcal{M}$ defined on some étale $U \rightarrow X$ that contains $\bar{x}$, let $\mu_{M}: \mathcal{O}_{X, \bar{x}} \rightarrow \mathcal{T}_{X, \bar{x}}^{1}$ denote the connecting homomorphism at $\bar{x}$ in the long exact sequence obtained from applying $\mathcal{H}$ om $\left(-, \mathcal{O}_{X}\right)$ to (5.1). By a general fact for extensions, we have $\mu_{M}(1)=\eta(M)$. For $\lambda \in \mathcal{O}_{X, \bar{x}}^{\times}$, let $M_{\lambda} \in \mathcal{L} \mathcal{S}_{X, \bar{x}}$ denote the element $\left(\mathcal{M}, \lambda^{-1} \tilde{\mathbb{1}}\right)$. The statement of the lemma comes down to the following claim.

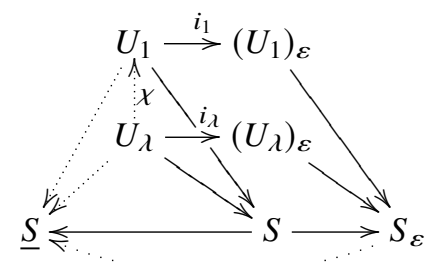


Claim 1. $\mu_{M}(\lambda)=\eta\left(M_{\lambda}\right)$.

To prove the claim, let $U_{1}, U_{\lambda}$ denote the $\log$ smooth schemes over $S$ respectively obtained from the $\log$ scheme $U$ and the map to $S$ given by $1 \mapsto \mathbb{1}$ and $1 \mapsto \lambda^{-1} \mathbb{1}$ respectively. Let $\left(U_{1}\right)_{\varepsilon}$ and $\left(U_{\lambda}\right)_{\varepsilon}$ be the unique deformations of $U_{1}, U_{\lambda}$ over $S_{\varepsilon}$, respectively. Let $\chi: U_{\lambda} \rightarrow U_{1}$ be the canonical isomorphism over $\underline{S}=\operatorname{Spec}(0 \rightarrow \mathbb{K})$.

We are now going to use facts about idealised log schemes; see [39, Chapter III.1.3 \& IV-Variant 3.1.21] for an introduction. We give $S_{\varepsilon}$ the ideal $\langle 2\rangle$ generated by $2 \in \mathbb{N}$ and $\left(U_{1}\right)_{\varepsilon}$ and $\left(U_{\lambda}\right)_{\varepsilon}$ the pullback ideals $K_{1}, K_{\lambda}$ respectively so that $\left(\left(U_{1}\right)_{\varepsilon}, K_{1}\right)$ and $\left(\left(U_{\lambda}\right)_{\varepsilon}, K_{\lambda}\right)$ are ideally log smooth over $\left(S_{\varepsilon},\langle 2\rangle\right)$. The map $\left(S_{\varepsilon},\langle 2\rangle\right) \rightarrow\left(A_{\mathbb{N}}, \emptyset\right)$ is an étale map of idealised log schemes and $A_{\mathbb{N}} \rightarrow \underline{S}$ is $\log$ smooth; hence, the composition $\pi:\left(\left(U_{1}\right)_{\varepsilon}, K_{1}\right) \rightarrow\left(S_{\varepsilon},\langle 2\rangle\right) \rightarrow \underline{S}$ is log smooth. We apply the infinitesimal lifting property to the diagram

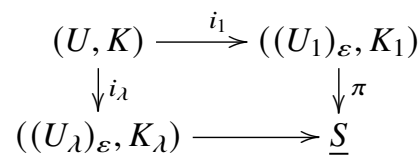

where $(U, K)$ is the idealised log scheme $U=U_{1} \stackrel{\chi}{=} U_{\lambda}$ with ideal given by $(\tilde{\mathbb{1}})^{2}$ or, equivalently, $\left(\lambda^{-1} \tilde{\mathbb{1}}\right)^{2}$. The left vertical map $i_{\lambda}$ is strict for the log structure and ideal and given by a square-zero ideal. We obtain a morphism $\tilde{\chi}:\left(U_{\lambda}\right)_{\varepsilon} \rightarrow\left(U_{1}\right)_{\varepsilon}$ of $\log$ schemes that preserves the ideals and is an isomorphism on ghost sheaves. Consequently, with $\rho_{\lambda} \in \mathcal{M}_{\left(U_{\lambda}\right)_{\varepsilon}, \bar{x}}$ and $\rho_{1} \in \mathcal{M}_{\left(U_{1}\right)_{\varepsilon}, \bar{x}}$, the images of the generator $1 \in \mathcal{M}_{S_{\varepsilon}}$ respectively, we have $\tilde{\chi}^{*} \rho_{1}=\tilde{\lambda} \cdot \rho_{\lambda}$ for some $\tilde{\lambda} \in \mathcal{O}_{\left(U_{\lambda}\right)_{\varepsilon}^{\times}, \bar{x}}$ that restricts to $\lambda \in \mathcal{O}_{U, \bar{x}}^{\times}$. This implies that $\tilde{\chi}$ becomes an isomorphism after shrinking $\left(U_{\lambda}\right)_{\mathcal{\varepsilon}},\left(U_{1}\right)_{\mathcal{\varepsilon}}$ if needed. Using $i_{1} \circ \chi=\tilde{\chi} \circ i_{\lambda}$, we obtain the commutative diagram

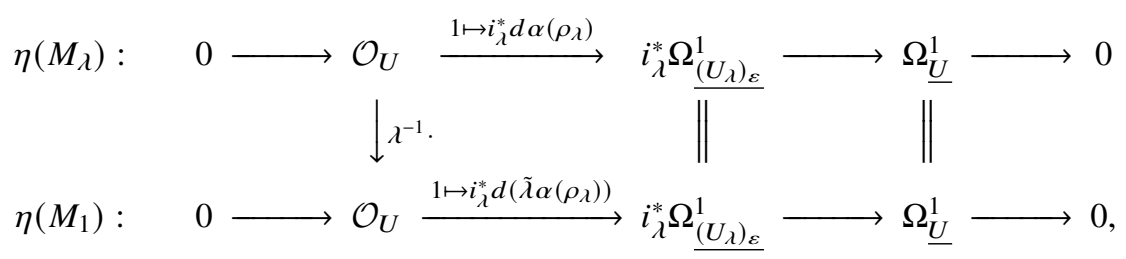

and we conclude $\eta\left(M_{\lambda}\right)=\mu_{M}(\lambda)$ via standard homological algebra.

Lemma 5.3. Let $\bar{x} \in X$ be a geometric point with $\mathbb{k}\left[\mathcal{P}_{\bar{x}}\right]$ smooth; then

1. for $M \in \mathcal{L S}_{X, \bar{x}}$, the map $\mu_{M, \bar{x}}: \mathcal{O}_{X, \bar{x}} \rightarrow \mathcal{T}_{X, \bar{x}}$ is surjective,

2. $\mathcal{O}_{X, \bar{x}}^{\times}$acts transitively on $\mathcal{L S}_{X, \bar{x}}$,

3. $\eta_{\bar{x}}: \mathcal{L} \mathcal{S}_{X, \bar{x}} \rightarrow \mathcal{T}_{X, \bar{x}}$ is injective.

Proof. Set $P:=\mathcal{P}_{\bar{x}}$. For (1), let $U \rightarrow X$ be an étale affine neighbourhood of $\bar{x}$ where $M=\left(\mathcal{M}_{U}, \mathbb{1}_{U}\right)$ is defined and $h:\left(U, \mathcal{M}_{U}\right) \rightarrow \operatorname{Spec}\left(P \rightarrow \mathbb{k}[P] /\left(z^{\mathbb{1}} \bar{x}\right)\right)$ the strict $S$-morphism whose underlying map is smooth. Possibly after shrinking $U$, via $\varepsilon \mapsto \mathbb{1}_{\bar{x}}$, we obtain a strict map of extensions over $S_{\varepsilon}$,

$$
h_{\varepsilon}:\left(U_{\varepsilon}, \mathcal{M}_{U_{\varepsilon}}\right) \rightarrow \operatorname{Spec}\left(P \rightarrow \mathbb{k}[P] /\left(z^{\left(\mathbb{1}_{\bar{x}}+\mathbb{1}_{\bar{x}}\right)}\right)\right)
$$

whose underlying morphism is also smooth and hence $\Omega_{\underline{U}_{\varepsilon}}$ is locally free. This implies that the corresponding term $\mathcal{E} x t^{1}\left(\Omega_{\underline{U_{\varepsilon}}}, \mathcal{O}_{X}\right)_{\bar{x}}$ in the long exact sequence for (5.1) vanishes and thus $\mu_{M, \bar{x}}$ is surjective.

To show (2), note that it suffices to show that any two elements in $\mathcal{L S}_{X, \bar{x}}$ are isomorphic over $\underline{S}$. Equivalently, by [19, Definition $3.19 \&$ Corollary 3.12], the composition

$$
\mathcal{L} \mathcal{S}_{X, \bar{x}} \subset \mathcal{E} x t^{1}\left(\mathcal{P}_{\bar{x}}^{\mathrm{gp}} / \mathbb{Z}_{\bar{x}}, \mathcal{O}_{X, \bar{x}}^{\times}\right) \rightarrow \mathcal{E} x t^{1}\left(\mathcal{P}_{\bar{x}}^{\mathrm{gp}}, \mathcal{O}_{X, \bar{x}}^{\times}\right)
$$


needs to be the constant map. By assumption, $P$ is free and then (2) follows from the description of $\mathcal{E} x t^{1}\left(\mathcal{P}_{\bar{x}}^{\mathrm{gp}}, \mathcal{O}_{X, \bar{x}}^{\times}\right)$in [19, Proposition 3.14].

For (3), if $\bar{x} \notin X_{\text {sing }}$, both stalks are trivial and there is nothing to show, so assume that $\bar{x} \in X_{\text {sing. }}$. By [17, Proposition 1.10], we have $\mathcal{T}_{X, \bar{x}}^{1} \cong \mathcal{O}_{X_{\text {sing }}, \bar{x}}$, so the kernel of the action of $\mathcal{O}_{X, \bar{x}}^{\times}$on $\mathcal{T}_{X, \bar{x}}^{1}$ is $K:=\operatorname{ker}\left(\mathcal{O}_{X, \bar{x}}^{\times} \rightarrow \mathcal{O}_{X_{\text {sing }}, \bar{x}}^{\times}\right)$. If we show that $K$ is contained in the kernel of the action of $\mathcal{O}_{X, \bar{x}}^{\times}$on $\mathcal{L} \mathcal{S}_{X, \bar{x}}$, then (3) follows from (2) and Proposition 5.2. By assumption, $X$ is normal crossings at $\bar{x}$. Let $X_{1}, \ldots, X_{r}$ be the local components of $X$ at $\bar{x}, r \geq 2$. Let $\lambda \in K$ be given and write $\lambda=1+\sum_{i=1}^{r} f_{i}$ where $\left.f_{i}\right|_{X_{j}}=0$ for $i \neq j$. We observe that $\lambda=\prod_{i}\left(1+f_{i}\right)$ because $f_{i} f_{j}=0$ for $i \neq j$. If $\mathbb{N}^{r} \rightarrow \mathcal{O}_{X, \bar{x}}, e_{i} \mapsto h_{i}$ is a chart of $X$ at $\bar{x}$ representing an element of $\mathcal{L} \mathcal{S}_{X, \bar{x}}$ with $\mathbb{1}=\sum_{i} e_{i}$ and $V\left(h_{i}\right)=X_{i}$, then $e_{i} \mapsto\left(1+f_{i}\right) e_{i}$ defines an automorphism of $\mathcal{M}_{X, \bar{x}}$ compatible with the map to $\mathcal{O}_{X, \bar{x}}$ because $\left(1+f_{i}\right) h_{i}=h_{i}$. It takes $\mathbb{1}$ to $\lambda \mathbb{1}$, so $\lambda$ acts trivially on $\mathcal{L} \mathcal{S}_{X, \bar{x}}$.

Remark 5.4. For $\kappa \geq 2$, consider the monoid $P_{\kappa}=\left\langle e_{1}, e_{2}, \mathbb{1} \mid e_{1}+e_{2}=\kappa \mathbb{1}\right\rangle$ and the toroidal crossing space $X=\operatorname{Spec}\left(P_{\kappa} \rightarrow \mathbb{k}\left[P_{K}\right] /\left(z^{\mathbb{1}}\right)\right)$. The map $\eta: \mathcal{L} \mathcal{S}_{X} \rightarrow \mathcal{T}_{X}^{1}$ is the zero map $\mathbb{k}^{\times} \rightarrow \mathbb{k}$, so the smoothness assumption in Lemma 5.3 is necessary.

Theorem 5.5. Let $X$ be a toroidal crossing space with $P_{\bar{x}} \cong \mathbb{N}^{2}$ whenever $\bar{x}$ is the generic point of a component of $X_{\text {sing; }}$; then $\eta: \mathcal{L} \mathcal{S}_{X} \rightarrow \mathcal{T}_{X}^{1}$ is injective. On the open set $V \subset X$ of points $\bar{x}$ with $\mathcal{P}_{\bar{x}} \cong \mathbb{N}^{r}$ for some $r$, we have $\eta\left(\mathcal{L S}_{V}\right)=\left(\mathcal{T}_{V}^{1}\right)^{\times}$where $\left(\mathcal{T}_{X}^{1}\right)^{\times} \subset \mathcal{T}_{X}^{1}$ denotes the subsheaf of those elements that generate $\mathcal{T}_{X}^{1}$ as an $\mathcal{O}_{X}$-module.

Proof. The second statement is Lemma 5.3. The first statement also follows from the lemma combined with the fact that for every open $U \subset X$, the restriction map $\mathcal{L S}_{X}(U) \rightarrow \mathcal{L S}_{X}(U \cap V)$ is injective, which is a consequence of Corollary 6.2. Indeed, in view of the diagram on the right, that the composition of the left vertical and bottom horizontal arrow is injective implies the injectivity of the top horizontal arrow.

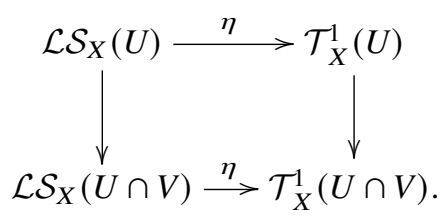

\section{Toroidal Crossing Spaces as Log Toroidal Families}

Let $X$ be a toroidal crossing space. Let $\bar{x}$ be geometric point and $V_{\bar{x}}$ the étale neighbourhood with a smooth map $V_{\bar{x}} \rightarrow \operatorname{Spec} \mathbb{K}\left[\mathcal{P}_{\bar{x}}\right] / z^{\mathbb{1}}$ that exists by the definition of $X$. Set $N=\mathcal{P}_{\bar{x}}^{\mathrm{gp}}$ and $M_{\mathbb{R}}=\operatorname{Hom}(N, \mathbb{R})$. We obtain a lattice polytope $\sigma_{\bar{x}}=\left\{m \in M_{\mathbb{R}}|m|_{\mathcal{P}_{\bar{x}}} \geq 0, \mathbb{1}(m)=1\right\}$ (we use that $X$ is reduced here). For a face $\tau \subset \sigma_{\bar{x}}$, we denote by $V_{\tau}$ the inverse image of the closed subset $\operatorname{Spec} \mathbb{k}\left[\tau^{\perp} \cap \mathcal{P}_{\bar{x}}\right]$ of Spec $\mathbb{k}\left[\mathcal{P}_{\bar{x}}\right] / z^{\mathbb{1}}$ in $V_{\bar{x}}$. Theorem 3.22 in [19] says the following.

Theorem 6.1 (Gross-Siebert). $\left.\mathcal{L} \mathcal{S}_{X}\right|_{V_{\bar{x}}}$ is isomorphic to a subsheaf of $\oplus_{\omega} \mathcal{O}_{V_{\omega}}^{\times}$where the sum is over the edges of $\sigma_{\bar{x}}$. The sections of the subsheaf on an open $V \subset V_{\bar{x}}$ are given as the tuples $\left(f_{\omega}\right)_{\omega}$ so that, for every two-face $\tau$ of $\sigma_{\bar{x}}$, we have

$$
\left.\prod_{\omega \subset \tau} d_{\omega} \otimes f_{\omega}^{\epsilon_{\tau}(\omega)}\right|_{V_{\tau}}=1
$$

as an equality in $M \otimes_{\mathbb{Z}} \Gamma\left(V, \mathcal{O}_{V_{\tau}}^{\times}\right)$where $d_{\omega}$ is a primitive generator of the tangent space to $\omega$ and $\epsilon_{\tau}(\omega) \in\{-1,+1\}$ is such that $\left(\epsilon_{\tau}(\omega) d_{\omega}\right)_{\omega \subset \tau}$ gives an oriented boundary of $\tau$.

Corollary 6.2. Given an étale open $U \rightarrow X$, the natural map $\mathcal{L S}_{X}(U) \rightarrow \prod_{\bar{x}} \mathcal{L S}_{X, \bar{x}}$, for the product running over the generic points $\bar{x}$ of the components of $U_{\text {sing, }}$, is injective. 
The isomorphism in the theorem naturally depends on the morphism $V_{\bar{x}} \rightarrow \operatorname{Spec} \mathbb{K}\left[\mathcal{P}_{\bar{x}}\right] / z^{\mathbb{1}}$ in a way that enables the following result.

Corollary 6.3. For each irreducible component $X_{\omega}$ of $X_{\text {sing }}$ there is an $\mathcal{O}_{\tilde{X}_{\omega}}^{\times}$-torsor $\mathcal{N}_{\omega}^{\times}$on its normalisation $\tilde{X}_{\omega}$ so that

$$
\mathcal{L S}_{X} \subset \bigoplus_{X_{\omega}} q_{\omega, *} \mathcal{N}_{\omega}^{\times}
$$

for $q_{\omega}: \tilde{X}_{\omega} \rightarrow X_{\omega}$ the normalisation, and the subsheaf is locally characterised by Theorem 6.1 when using suitable local trivialisations of the torsors.

Let $\mathcal{N}_{\omega}$ denote the associated line bundle so that $\mathcal{N}_{\omega}^{\times}$is its $\mathcal{O}_{\tilde{X}_{\omega}}^{\times}$-torsor of generating sections. We therefore obtain an injection of $\mathcal{L} \mathcal{S}_{X}$ in the coherent sheaf $\bigoplus_{X_{\omega}} q_{\omega, *} \mathcal{N}_{\omega}$.

Lemma 6.4. Under the hypothesis of Theorem 5.5, the injection $\mathcal{L} \mathcal{S}_{X} \hookrightarrow \bigoplus_{X_{\omega}} q_{\omega, *} \mathcal{N}_{\omega}$ is $\mathcal{O}_{X}^{\times}$equivariant.

Proof. We borrow the notation $P_{K}$ from Remark 5.4. From a careful analysis of the proof of [19, Theorem 3.22] one finds that the action $\mathbb{1} \mapsto \lambda^{-1} \mathbb{1}$ becomes $f_{\omega} \mapsto \lambda^{\kappa_{\omega}} f_{\omega}$ where $\kappa_{\omega}$ is such that $\mathcal{P}_{\bar{x}} \cong P_{\kappa_{\omega}}$ at the generic point $\bar{x}$ of $X_{\omega}$. Indeed, if a local model at $\bar{x}$ is given by $x y=f_{\omega}\left(z^{\mathbb{1}}\right)^{\kappa_{\omega}}$, this is equivalent to $x y=\lambda^{\kappa_{\omega}} f_{\omega}\left(\lambda^{-1} z^{\mathbb{1}}\right)^{\kappa_{\omega}}$, which explains the action. The hypothesis of Theorem 5.5 says that $\kappa_{\omega}=1$ for all $\omega$, so indeed the action of $\mathcal{O}_{X}^{\times}$on $\mathcal{L} \mathcal{S}_{X}$ is compatible with the ordinary action on the coherent sheaf $\bigoplus_{X_{\omega}} q_{\omega, *} \mathcal{N}_{\omega}$.

Theorem 6.5. If $X$ is a normal crossing space, then the injection in Lemma 6.4 factors as the composition of $\eta: \mathcal{L} \mathcal{S}_{X} \rightarrow \mathcal{T}_{X}^{1}$ and a uniquely determined injection of coherent sheaves $\mathcal{T}_{X}^{1} \hookrightarrow \bigoplus_{X_{\omega}} q_{\omega, *} \mathcal{N}_{\omega}$.

Proof. Given Lemma 6.4 and Theorem 5.5 and noting that $V=X$ for a normal crossing space and that the annihilator of $\mathcal{T}_{X}^{1}$ is contained in the annihilator of $\bigoplus_{X_{\omega}} q_{\omega, *} \mathcal{N}_{\omega}$, the statement becomes an elementary lemma about a cyclic module whose proof we omit.

Definition 6.6. For a point $\bar{x} \in X$, let $X_{\bar{x}}^{\circ} \subset X$ denote the Zariski locally closed subset where $\mathcal{P}$ is locally constant with stalk $\mathcal{P}_{\bar{x}}$, so that $X$ is the disjoint union of $X_{\bar{y}}^{\circ}$ for suitable points $\bar{y}$. We call the closure $X_{\bar{x}}$ of $X_{\bar{x}}^{\circ}$ the stratum of $\bar{x}$, which again decomposes into $X_{\bar{y}}^{\circ}$. We infer the notion of strata to the normalisation of $X$.

A section of $s \in \Gamma\left(U, \mathcal{L S}_{X}\right)$ for a Zariski open $U \subset X$ is called schön if it extends to a section $\left(s_{\omega}\right)_{\omega} \in \Gamma\left(X, \bigoplus_{X_{\omega}} q_{\omega, *} \mathcal{N}_{\omega}\right)$ so that, for each $\omega$, the vanishing locus $\tilde{Z}_{\omega}$ of $s_{\omega}$ in $\tilde{X}_{\omega}$ is reduced, does not contain any strata and has regular intersection with each stratum inside $\tilde{X}_{\omega}$ (in particular, $\tilde{Z}_{\omega} \cap X_{\omega}^{\circ}$ is smooth). We also assume that $Z=\bigcup_{\omega} q_{\omega}\left(\tilde{Z}_{\omega}\right)$ is the complement of $U$ (otherwise, $U$ can be enlarged).

Definition 6.7. A schön section is called simple if for every closed point $\bar{x} \in X$ with $V_{\bar{x}} \rightarrow$ Spec $\mathbb{k}\left[\mathcal{P}_{\bar{x}}\right] / z^{\mathbb{1}}$ the smooth map from a neighbourhood, we have the following situation. Let $Z \cap V_{\bar{x}}=$ $\bigcup_{\omega \in \Omega} Z_{\omega}$ be the local decomposition of $Z$ into irreducible components where we may assume each $Z_{\omega}$ contains $\bar{x}$.

1. There is a disjoint union $\Omega=\Omega_{1} \sqcup \ldots \sqcup \Omega_{q}$ with $q<\operatorname{rk} \mathcal{P}_{\bar{x}}$ such that $Z_{i}:=Z_{\omega} \cap X_{\bar{x}}=Z_{\omega^{\prime}} \cap X_{\bar{x}}$ whenever $\omega, \omega^{\prime}$ are in the same $\Omega_{i}$.

2. $Z_{1}, \ldots, Z_{q}$ form a collection of normal crossing divisors in $X_{\bar{x}}$ at $\bar{x}$.

3. For each $i$, the primitive vectors $d_{\omega}$ for $\omega \in \Omega_{i}$ are the set of edge vectors of an elementary simplex $\Delta_{i} \subset N_{\mathbb{R}}$. (A lattice simplex is elementary if its vertices are the only lattice points contained in it.)

We remark that if $q_{\omega}: \tilde{X}_{\omega} \rightarrow X_{\omega}$ is not an embedding, the zero set $\tilde{Z}_{\omega}$ of $s_{\omega}$ may locally contribute two or more components of $Z$ at a point $\bar{x}$ that may or may not lie in different $\Omega_{i}$. 
Theorem 6.8 (Gross-Siebert). A toroidal crossing space X over an algebraically closed field $\mathbb{k}$ together with simple section $s \in \Gamma\left(U, \mathcal{L} \mathcal{S}_{X}\right)$ gives $X$ the structure of a log toroidal family over $S=\operatorname{Spec}(\mathbb{N} \rightarrow \mathbb{k})$ with $U$ the locus of log smoothness.

Proof. Using assumptions in Definition 6.7, the proof is the same as the one of [20, Theorem 2.6]. See also Example 4.6.

We remark that the $\Delta_{i}$ give the local structure of the singularities in the nearby fibre (cf. [20, Proposition 2.2]). We also remark that all ETDs have $\mathcal{F}=\mathcal{F}_{\min }$; that is, there is no horizontal divisor. Proposition 2.8 implies $W_{X / S}^{\operatorname{dim} X}=\omega_{X / S}$.

Proposition 6.9. A normal crossing space $X$ with $X_{\text {sing }}$ projective and $\mathcal{T}_{X}^{1}$ generated by global sections permits a dense open $U$ and a simple section $s \in \Gamma\left(U, \mathcal{L} \mathcal{S}_{X}\right)$. In view of Definition 6.7, we have $q=1$ at every point in $Z$ and $\Delta_{1}$ in each ETD is a standard simplex, which means that all ETDs have smooth nearby fibres.

Proof. Applying Bertini's theorem to the line bundle $\mathcal{T}_{X}^{1}$ on $X_{\text {sing }}$, we obtain a section $\hat{s} \in \Gamma\left(X_{\text {sing }}, \mathcal{T}_{X}^{1}\right)$ that gives a simple section $s \in \Gamma\left(X \backslash V(\hat{s}), \mathcal{L S}_{X}\right)$ by Theorem 6.5.

Proposition 6.10. Theorem 1.1 follows from Theorem 1.7.

Proof. We are given $E$ that is transverse to the strata of $X$. We apply a slight variant of Proposition 6.9 by making sure that the zero locus $Z$ of the section $\hat{s}$ generated by Bertini is transverse also to $E$. Theorem 1.7 gives an orbifold smoothing, but we know that it is an actual smoothing from the fact that each $\Delta_{1}$ is standard.

The next two lemmata reduce Theorem 1.7 to the log Calabi-Yau case; that is, to the case $W_{X / S}^{d} \cong \mathcal{O}_{X}$. We achieve this by modifying the log structure so that the new family is log Calabi-Yau.

Lemma 6.11. Let $f: X \rightarrow S$ be a log toroidal family with empty horizontal divisor. Let $E \subset \underline{X}$ be a Cartier divisor that meets all strata and $Z$ transversely; that is, locally along $E$ the triple $(X, Z, E)$ is étale equivalent to $\left(E \times \mathbb{A}^{1},(E \cap Z) \times \mathbb{A}^{1}, E \times\{0\}\right)$. There is a new $\log$ toroidal family $X(\log E) \rightarrow S$ that has $E$ as its horizontal divisor and factors through $f$ (by forgetting $E$ ), so, in particular, $W_{X}^{\operatorname{dim} X}(\log E) / S(-E)=$ $\omega_{X / S}$.

Proof. On $U$ the result is straightforward and along $Z$ we use the product description to make $E$ the horizontal divisor in the ETDs by adding a summand $\mathbb{N}$ to $P$ and the unique new facet gets included in $\mathcal{F}$. That these give local models follows the same proof as [20, Theorem 2.6], noting that we may treat the local equation for $E$ as one of the $f_{i}$ in the notation of [20].

Lemma 6.12. Let $f: X \rightarrow S$ be a projective log toroidal family with empty horizontal divisor and assume that $\omega_{X / S}^{-1}$ is generated by global sections; then $\omega_{X / S}^{-1} \cong \mathcal{O}_{X}(E)$ for a divisor E that satisfies the assumption of Lemma 6.11. In particular, $W_{X(\log E) / S}^{\operatorname{dim} X} \cong \mathcal{O}_{X}$.

Proof. This follows via an application of Bertini's theorem.

In general, we do not know whether deformations of log toroidal families are locally unique. The following theorem shows local uniqueness for the families obtained from toroidal crossing spaces whenever a simple section gives the log structure.

Theorem 6.13 (Gross-Siebert, Theorem 2.11 in [20]). Let $Y:=X(\log E) \rightarrow S$ be a $\log$ toroidal family obtained from a toroidal crossing space $\underline{X}$ via a simple section $s \in \Gamma\left(U, \mathcal{L S}_{\underline{X}}\right)$ and a divisor $E$ as in Lemma 6.11. Let $Y_{k}$ be a log toroidal deformation over $S_{k}=\operatorname{Spec}\left(\mathbb{N} \rightarrow \mathbb{k}[t] /\left(t^{k+1}\right)\right)$. Then the automorphisms of, isomorphisms of and obstructions to the existence of a lifting $Y_{k+1}$ to $S_{k+1}$ are controlled by $H^{0}\left(Y, \Theta_{Y / S}^{1} \otimes_{\mathbb{R}} I\right), H^{1}\left(Y, \Theta_{Y / S}^{1} \otimes_{\mathbb{K}} I\right)$ and $H^{2}\left(Y, \Theta_{Y / S}^{1} \otimes_{\mathbb{R}} I\right)$, respectively, where $I=\left(t^{k+1}\right) \subset \mathbb{k}[t] /\left(t^{k+2}\right)$. In particular, if $V \subset Y$ is affine open, then any two infinitesimal deformations of $V / S$ are isomorphic. 
Proof. The proof works precisely as in [20]. We remark that in Lemma 2.14, the exact sequence in (2) becomes $0 \rightarrow \Theta_{Y / S} \rightarrow \Theta_{X / \mathbb{k}}(\log E) \rightarrow \mathcal{B} \rightarrow 0$ where $\Theta_{X / \mathbb{k}}(\log E)$ denotes ordinary derivations that preserve the ideal of $E$. In other words, for the ordinary deformations, we consider the ones of the pair $(\underline{X}, E)$ rather than just $\underline{X}$.

\section{Differentials for Elementary Log Toroidal Families}

We fix a principal ideal domain $R$ as base ring. The constructions in Section 3 carry through when replacing $\mathbb{Z}$ by $R$. We will use the following elementary lemma.

Lemma 7.1. Let $n, m \geq 0$ and $G_{1}, \ldots, G_{r} \subset R^{n}$ be submodules each of which is a direct summand; then the natural map $\bigwedge_{R}^{m}\left(\bigcap_{i} G_{i}\right) \rightarrow \bigcap_{i} \bigwedge_{R}^{m} G_{i}$ is an isomorphism.

First consider the absolute case - that is, an $\operatorname{ETD}(Q \subset P, \mathcal{F})$ with $Q=0-$ and let $f: A_{P, \mathcal{F}} \rightarrow$ Spec $R$ be the associated $\log$ morphism. One checks that $U$ from (3.3) is simply the complement of codimension 2 strata. Recall from Example 2.10 that $W^{m}:=W_{A_{P, \mathcal{F}} / \operatorname{Spec} R}^{m}$ are just the Danilov differentials with $\log$ poles in the divisor given by the facets in $\mathcal{F}$. Danilov already computed these in [12, Proposition 15.5] over a field, and because of Lemma 7.1 the same calculation works over $R$ and we obtain the following.

Proposition 7.2 (absolute case). We have a grading $\Gamma\left(A_{P}, W^{m}\right)=\bigoplus_{p \in P}\left(W^{m}\right)_{p}$ with

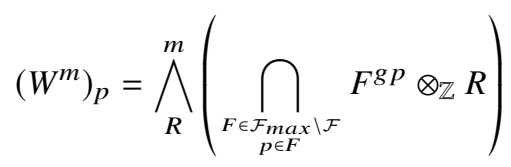

where the intersection is $P^{g p} \otimes_{\mathbb{Z}} R$ if the index set is empty.

Let us next assume that we have a general $\operatorname{ETD}(Q \subset P, \mathcal{F})$ and let $f$ again denote the associated $\log$ toroidal family and $W_{f}^{m}:=W_{A_{P, \mathcal{F}} / \operatorname{Spec} A_{Q}}^{m}$ the differentials. Note that because $\mathcal{F}$ contains all vertical facets, every facet in $\mathcal{F}_{\max } \backslash \mathcal{F}$ contains $Q$. We obtain the following generalisation.

Proposition 7.3 (general case). We have a grading $\Gamma\left(A_{P}, W_{f}^{m}\right)=\bigoplus_{p \in P}\left(W_{f}^{m}\right)_{p}$ with

$$
\left(W_{f}^{m}\right)_{p}=\bigwedge_{R}^{m}\left(\left(\bigcap_{\substack{F \in \mathcal{F}_{\max } \backslash \mathcal{F} \\ p \in F}} F^{g p} \otimes_{\mathbb{Z}} R\right) /\left(Q^{g p} \otimes_{\mathbb{Z}} R\right)\right)
$$

where the intersection is $P^{g p} \otimes_{\mathbb{Z}} R$ if the index set is empty. Because $Q^{g p} \subset P^{g p}$ splits, we can equivalently take the quotient before the intersection.

Proof. We can compose $f$ with the projection to $\operatorname{Spec} R$ to relate the current situation to that of Proposition 7.2. The open set $U^{\text {abs }}$ in the absolute case is the complement of $Z^{\text {abs }}$, the union of all codimension 2 strata. Hence, $U^{\text {abs }}$ is covered by $U_{F}$ where $F$ runs over the facets of $P$. On the other hand, the open set $U$ for $f$ as given in (3.3) has a cover $U_{F}$ where $F$ runs over the essential faces of rank $d-1$ by Lemma 3.7. Obviously, $U^{\text {abs }} \subset U$. Note that because $W_{f}^{m}$ is locally free on $U$ and $\mathcal{O}_{U}$ is $Z^{\text {abs }}$-closed, we find that $W_{f}^{m}$ is not only $Z$-closed but also $Z^{\text {abs }}$-closed. Consider the commutative diagram of solid arrows

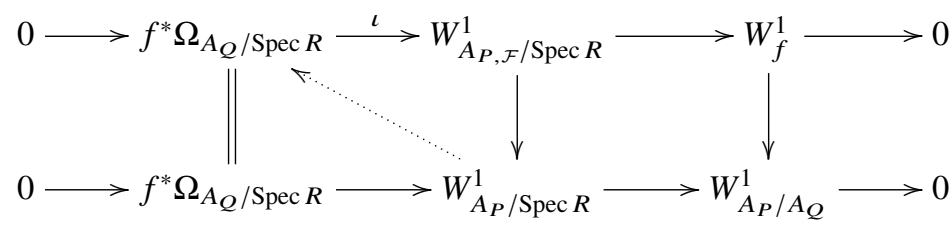


where the top row is obtained by pushing it forward from $U^{\text {abs }}$. The bottom sequence is obtained from tensoring the sequence $0 \rightarrow Q^{\mathrm{gp}} \rightarrow P^{\mathrm{gp}} \rightarrow P^{\mathrm{gp}} / Q^{\mathrm{gp}} \rightarrow 0$ with $\mathcal{O}_{A_{P}}$; in particular, it is exact and splits. Hence, the dotted diagonal arrow exists and commutes with the other maps. Therefore, $\operatorname{coker}(\iota)$ is a direct summand of $W_{A_{P, \mathcal{F}} / \operatorname{Spec} R}^{1}$; in particular, $Z^{\text {abs }}$-closed. Moreover, $\operatorname{coker}(\iota) \rightarrow W_{f}^{1}$ is an isomorphism on $U^{\text {abs }}$ and because both sheaves are $Z^{\text {abs }}$-closed, we have coker $(\iota)=W_{f}^{1}$ and thus the top row is exact and splits.

Let $\left\langle f^{*} \Omega_{A_{Q} / \operatorname{Spec} R}\right\rangle$ denote the homogeneous ideal in the sheaf of exterior algebras $W_{A_{P, \mathcal{F}} / \operatorname{Spec} R}^{\bullet}$ generated by $f^{*} \Omega_{A_{Q} / \operatorname{Spec} R}$. The split exactness above gives the split exactness of the following sequence:

$$
0 \rightarrow\left\langle f^{*} \Omega_{A_{Q} / \operatorname{Spec} R}\right\rangle_{m} \rightarrow W_{A_{P, \mathcal{F}} / \operatorname{Spec} R}^{m} \rightarrow W_{f}^{m} \rightarrow 0 .
$$

Because $A_{P}$ is affine and $\left\langle f^{*} \Omega_{A_{Q} / \text { Spec } R}\right\rangle$ coherent, applying $\Gamma\left(A_{P}, \cdot\right)$ to this sequence yields another exact sequence that already gives that $\Gamma\left(A_{P}, W_{f}^{m}\right)$ is $P$-graded. We have $\Gamma\left(A_{P}, f^{*} \Omega_{A_{Q} / \operatorname{Spec} R}\right)=Q^{\mathrm{gp}} \otimes_{\mathbb{Z}}$ $R[P]$. Set $\mathbf{F}_{p}:=\left(\bigcap_{\substack{F \in \mathcal{F} \max \backslash \mathcal{F} \\ p \in F}} F^{g p} \otimes_{\mathbb{Z}} R\right)$ and let $\left\langle Q^{g p} \otimes R\right\rangle \subset \wedge_{R}^{\bullet} \mathbf{F}_{p}$ be the homogeneous ideal generated by $Q^{\mathrm{gp}} \otimes R$. One computes $\Gamma\left(A_{P},\left\langle f^{*} \Omega_{A_{Q} / \text { Spec } R}\right\rangle_{m}\right)_{p}=\left\langle Q^{\mathrm{gp}} \otimes R\right\rangle_{m}$. Using Proposition 7.2, in degree $p \in P$, we obtain the exact sequence

$$
0 \rightarrow\left\langle Q^{\mathrm{gp}} \otimes R\right\rangle_{m} \rightarrow \bigwedge_{R}^{m} \mathbf{F}_{p} \rightarrow\left(W_{f}^{m}\right)_{p} \rightarrow 0 .
$$

Using a splitting of the injection $\left(Q^{\mathrm{gp}} \otimes R\right) \subset \mathbf{F}_{p}$ and comparing leads to the assertion.

Corollary 7.4. For all $m, W_{f}^{m}$ is flat over $A_{Q}$.

Proof. Inspecting the result in Proposition 7.3, we find that $\Gamma\left(A_{P}, W_{f}^{m}\right)$ is a free $R[Q]$-module.

\subsection{Change of Base}

Let $(Q \subset P, \mathcal{F})$ be an ETD, $\mathcal{T}$ be a Noetherian ring and $T=\operatorname{Spec} \mathcal{T} \rightarrow \operatorname{Spec} R[Q]$ be any morphism. Denote by $\sigma$ the composition $Q \rightarrow R[Q] \rightarrow \mathcal{T}$, which turns $T$ into a coherent log scheme. Define $Y$ by the fibre diagram

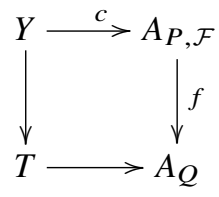

of $\log$ toroidal families. We want to study when the natural map $c^{*} W_{f}^{m} \rightarrow W_{Y / T}^{m}$ is an isomorphism. This holds if $f$ is $\log$ smooth because then $W_{f}^{m}=\Omega_{f}^{m}$ are the ordinary log differentials that satisfy this isomorphism property by their universal property. In particular, $c^{*} W_{f}^{m} \rightarrow W_{Y / T}^{m}$ is always an isomorphism on the open set $V:=c^{-1}(U)$. The following example shows that it is not an isomorphism in general. For a subset $I \subset P$, let $\langle I\rangle$ be the smallest face of $P$ containing $I$.

Example 7.5. Let $P$ be the submonoid of $\mathbb{Z}^{2}$ generated by $(1,0),(1,1),(1,2)$ and let $Q=0$. The monoid $P$ has two facets $H_{1}=\langle(1,0)\rangle$ and $H_{2}=\langle(1,2)\rangle$, and setting $\mathcal{F}=\emptyset$ yields an ETD. Let $f: A_{P, \mathcal{F}} \rightarrow A_{Q}=$ Spec $\mathbb{Z}$ be the corresponding map. Now set $\mathcal{T}=\mathbb{Z} / 2 \mathbb{Z}$ inducing the natural map $T=\operatorname{Spec} \mathcal{T} \rightarrow \operatorname{Spec} \mathbb{Z}$ and a fibre diagram as above. One checks that $c^{*} W_{f}^{1} \rightarrow W_{Y / T}^{1}$ is not an isomorphism by computing both terms via Proposition 7.2. It suffices to check the degree $p=0-$ indeed, $\left(W_{f}^{1}\right)_{0}=H_{1}^{\mathrm{gp}} \cap H_{2}^{\mathrm{gp}}=0-$ but

$$
\left(W_{Y / T}^{1}\right)_{0}=\left(H_{1}^{\mathrm{gp}} \otimes \mathbb{Z} / 2 \mathbb{Z}\right) \cap\left(H_{2}^{\mathrm{gp}} \otimes \mathbb{Z} / 2 \mathbb{Z}\right)=\mathbb{Z} / 2 \mathbb{Z} \cdot(1,0) \subset(\mathbb{Z} / 2 \mathbb{Z})^{2} .
$$

Hence, $\left(\left(W_{f}^{1}\right) \otimes_{\mathbb{Z}} \mathbb{Z} / 2 \mathbb{Z}\right)_{0}=0$ but $\left(W_{Y / T}^{1}\right)_{0} \neq 0$. 
The example teaches that base change is related to the (non)commuting of intersection and tensor product. The following lemma (which is an elementary exercise in Tor groups) will help us. We say that a ring $\mathcal{T}$ is of characteristic $\geq p_{0}$ if for the residue field $\kappa_{\mathfrak{p}}$ of every point $\mathfrak{p}$ holds char $\kappa_{\mathfrak{p}} \geq p_{0}$ or $\operatorname{char} \kappa_{\mathfrak{p}}=0$.

Lemma 7.6. Let $G$ be a finitely generated $\mathbb{Z}$-module and $H, H^{\prime} \subset G$ be two submodules. Then there is $p_{0}$ such that for every ring $\mathcal{T}$ of characteristic $\geq p_{0}$ we have

$$
\left(H \cap H^{\prime}\right) \otimes \mathcal{T}=(H \otimes \mathcal{T}) \cap\left(H^{\prime} \otimes \mathcal{T}\right)
$$

and each term here is a submodule of $G \otimes \mathcal{T}$.

In the general situation, observe that we have $\Gamma\left(Y, \mathcal{O}_{Y}\right)=\bigoplus_{e \in E} z^{e} \cdot \mathcal{T}$ with multiplication

$$
z^{e_{1}} \cdot z^{e_{2}}=z^{e} \cdot \sigma(q) \text { whenever } e_{1}+e_{2}=e+q
$$

with $e \in E, q \in Q$ under the canonical decomposition from (3.1). Similarly, Proposition 7.3 gives

$$
\Gamma\left(Y, c^{*} W_{f}^{m}\right)=\bigoplus_{e \in E} z^{e} \cdot\left(\left(W_{f}^{m}\right)_{e} \otimes_{R} \mathcal{T}\right)
$$

Lemma 7.7. Recall $V=c^{-1}(U)$. Equivalent are

1. the $\operatorname{map}^{*} W_{f}^{m} \rightarrow W_{Y / T}^{m}$ is an isomorphism,

2. $c^{*} W_{f}^{m}$ is reflexive,

3. the restriction map $\rho: \Gamma\left(Y, c^{*} W_{f}^{m}\right) \rightarrow \Gamma\left(V, c^{*} W_{f}^{m}\right)$ is surjective.

Proof. (1) $\Rightarrow(2): W_{Y / T}^{m}$ is reflexive; (2) $\Rightarrow(3): c^{*} W_{f}^{m}$ is $(Y \backslash V)$-closed; (3) $\Rightarrow(1)$ : Consider the commutative square

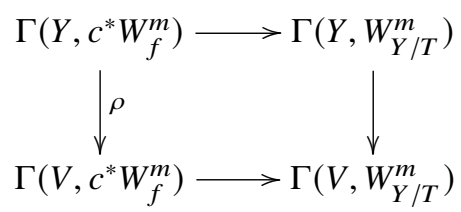

where the right vertical map is an isomorphism because $W_{Y / T}^{m}$ is reflexive by Lemma 2.4. The bottom horizontal map is an isomorphism by what we said just before Example 7.5. Now (1) holds if the top horizontal map is an isomorphism, which follows from (3) if $\rho$ is additionally injective. This injectivity is a general fact that we prove next. Recall that $A_{P, \mathcal{F}_{\max }}=A_{P}$ and we have a map $A_{P} \rightarrow A_{P, \mathcal{F}}$ that gives us another commutative square

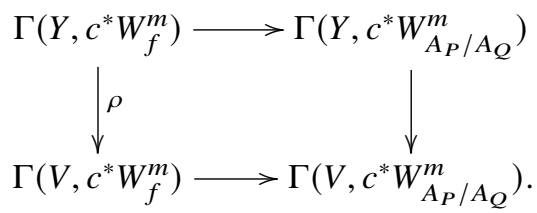

Because $A_{P} \rightarrow A_{Q}$ is $\log$ smooth and $W_{A_{P} / A_{Q}}^{m}=\Omega_{A_{P} / A_{Q}}^{m}$ a free sheaf, the right vertical map is an isomorphism. We get that $\rho$ is injective if the top horizontal map is injective. The latter can be computed from Proposition 7.3. Indeed, this follows from (7.3) because for every $e \in E$, the cokernel of $\left(W_{f}^{m}\right)_{e} \rightarrow\left(W_{A_{P} / A_{Q}}^{m}\right)_{e}$ is a free $R$-module. 
We next provide a useful criterion for the surjectivity of $\rho$. Let $\mathcal{E}$ be the set of essential faces of $P$ of rank $d-1$. By Lemma 3.7, $U$ is covered by $\left\{U_{F} \mid F \in \mathcal{E}\right\}$. Set $V_{F}=c^{-1}\left(U_{F}\right)$ so these cover $V$. For each $F \in \mathcal{E}$, choose $e_{F} \in F$ in the relative interior; that is, $\left\langle e_{F}\right\rangle=F$.

Theorem 7.8. Write $M_{p}:=\left(W_{f}^{m}\right)_{p}$ for short and assume that for every subset $\mathcal{E}^{\prime} \subset \mathcal{E}$ and every $e \in E$ the natural map

$$
\left(\bigcap_{F \in \mathcal{E}^{\prime}} M_{e+e_{F}}\right) \otimes_{R} \mathcal{T} \rightarrow \bigcap_{F \in \mathcal{E}^{\prime}}\left(M_{e+e_{F}} \otimes_{R} \mathcal{T}\right)
$$

is an isomorphism. Then $\rho$ is surjective.

Proof. We write $M=\Gamma\left(A_{P}, W_{f}^{m}\right), N=\Gamma\left(A_{P}, W_{A_{P} / A_{Q}}^{m}\right)$ and $N_{p}$ for the degree $p$ part of $N$. By proposition 7.3, $M_{p}$ and $N_{p}$ only depend on $\langle p\rangle$. We are going to use that for $p_{1}, p_{2} \in P$ holds

$$
\left\langle p_{1}+p_{2}\right\rangle=\left\langle\left\langle p_{1}\right\rangle \cup\left\langle p_{2}\right\rangle\right\rangle .
$$

We have a natural injection $M \subset N$ by Proposition 7.3. Given $\mu \in \Gamma\left(V, c^{*} W_{f}^{m}\right)$, we want to show that it has a preimage under $\rho$. We do have a unique preimage $v$ under the right vertical map of (7.4), so in $N \otimes_{R[Q]} \mathcal{T}$, and we are going to show that this preimage lies in $M \otimes_{R[Q]} \mathcal{T}$. Say $v=\sum_{e} z^{e} \cdot n_{e}$ with $n_{e} \in N_{e} \otimes \mathcal{T}$ is such that $\left.v\right|_{V}=\mu$. In particular, $\left.v\right|_{V_{F}}=\left.\mu\right|_{V_{F}}$ for all $F \in \mathcal{E}$. There is some large $a \geq 1$ so that for each $F \in \mathcal{E}$ there are $m_{F, e} \in M_{e} \otimes \mathcal{T}$ such that

$$
\left.\mu\right|_{V_{F}}=z^{-a e_{F}} \sum_{e} z^{e} \cdot m_{F, e}
$$

and therefore $\left.v\right|_{V_{F}}=\left.\mu\right|_{V_{F}}$ implies

$$
z^{a e_{F}} \sum_{e} z^{e} \cdot n_{e} \in \bigoplus_{e \in E} z^{e} \cdot\left(M_{e} \otimes_{R} \mathcal{T}\right) \subset \bigoplus_{e \in E} z^{e} \cdot\left(N_{e} \otimes_{R} \mathcal{T}\right) .
$$

If $e+a e_{F}=\tilde{e}+q$ is the decomposition $P=E \times Q$, then $n_{e} \cdot \sigma(q) \in M_{\tilde{e}} \otimes_{R} \mathcal{T}$. By (7.5),

$$
e+a e_{F} \in E \Longleftrightarrow\left\langle e+e_{F}\right\rangle \subset E \Longleftrightarrow e+e_{F} \in E,
$$

and if this holds, then $\sigma(q)=1$, so setting

$$
\mathcal{E}_{e}:=\left\{F \in \mathcal{E} \mid e+e_{F} \in E\right\},
$$

we obtain $n_{e} \in \bigcap_{F \in \mathcal{E}_{e}}\left(M_{e+a e_{F}} \otimes_{R} \mathcal{T}\right)$ and $M_{e+a e_{F}}=M_{e+e_{F}}$. Note that $\mathcal{E}_{e}$ does not depend on the chosen $e_{F}$. Using the assumption, we get

$$
n_{e} \in \bigcap_{F \in \mathcal{E}_{e}}\left(M_{e+e_{F}} \otimes_{R} \mathcal{T}\right)=\left(\bigcap_{F \in \mathcal{E}_{e}} M_{e+e_{F}}\right) \otimes_{R} \mathcal{T}
$$

For the next step, define $\mathcal{F}_{e}=\left\{H \in \mathcal{F}_{\text {max }} \backslash \mathcal{F} \mid \exists F \in \mathcal{E}_{e}: e+e_{F} \in H\right\}$. We use Lemma 7.1 to compute

$$
\bigcap_{F \in \mathcal{E}_{e}} M_{e+e_{F}}=\bigwedge_{R}^{m}\left(\bigcap_{H \in \mathcal{F}_{e}} \frac{H^{g p} \otimes_{\mathbb{Z}} R}{Q^{g p} \otimes_{\mathbb{Z}} R}\right) .
$$

We finally claim that $\mathcal{F}_{e}=\left\{H \in \mathcal{F}_{\text {max }} \backslash \mathcal{F} \mid e \in H\right\}$; indeed, given an $H$ in the latter, we just need to exhibit an $F \in \mathcal{E}$ that is also contained in $H$ with $\langle e, F\rangle \subset E$, which can be done because $H \cap E$ is a union of faces in $\mathcal{E}$. Thus, $n_{e} \in M_{e} \otimes_{R} \mathcal{T}$, so indeed $v \in M \otimes_{R}[Q] \mathcal{T}$ and we are done. 
Corollary 7.9. Let $(Q \subset P, \mathcal{F})$ be an ETD, $\mathcal{T}$ a Noetherian ring and $T=\operatorname{Spec} \mathcal{T} \rightarrow A_{Q}$ a strict morphism of log schemes. Then $c^{*} W_{f}^{m}$ is reflexive and $c^{*} W_{f}^{m} \rightarrow W_{Y / T}^{m}$ is an isomorphism provided that the composition

$$
R \rightarrow R[Q] \rightarrow \mathcal{T}
$$

is flat; for example, when $R$ is a field.

As Example 7.5 shows, the conditions of Theorem 7.8 are not always satisfied in case $R=\mathbb{Z}$. However, we do get close.

Corollary 7.10. Let $(Q \subset P, \mathcal{F})$ be an ETD, and assume $f: A_{P, \mathcal{F}} \rightarrow A_{Q}$ to be defined over $R=\mathbb{Z}$. Then there is a $p_{0}=p_{0}(Q \subset P, \mathcal{F})$ such that for every $m$ and every $T=\operatorname{Spec} \mathcal{T} \rightarrow A_{Q}$ with a Noetherian ring $\mathcal{T}$ of characteristic $\geq p_{0}$, the sheaf $c^{*} W_{f}^{m}$ is reflexive, and $c^{*} W_{f}^{m} \rightarrow W_{Y / T}^{m}$ is an isomorphism.

Proof. Applying Lemma 7.6 repeatedly, we find for every triple $\left(m, e, \mathcal{E}^{\prime}\right)$ as in Theorem 7.8 a $p_{0}\left(m, e, \mathcal{E}^{\prime}\right)$ such that the isomorphism in the theorem holds if $\mathcal{T}$ is of characteristic $\geq p_{0}\left(m, e, \mathcal{E}^{\prime}\right)$. Because there are only finitely many different sets of modules $\left\{M_{e+e_{F}} \mid F \in \mathcal{E}^{\prime}\right\}$, we obtain one $p_{0}(Q \subset P, \mathcal{F})$ that works for all triples.

For a field $\mathbb{k}$, consider a monoid ideal $K \subset Q$, let $(K) \subset \mathbb{k}[Q]$ denote the corresponding monomial ideal of $\mathbb{k}[Q]$ and set $\mathcal{T}=\mathbb{k}[Q] /(K)$. The map $T=\operatorname{Spec} \mathcal{T} \rightarrow \mathbb{k}[Q]$ is the natural one and $Y \rightarrow T$ is defined by (7.2) as before. We set $E_{K}:=P \backslash(P+K)$ and note this generalises the union of essential faces $E$ from Section 3; indeed, $E=E_{Q \backslash\{0\}}$. Combining Proposition 7.3 with Corollary 7.9 (for $R=\mathbb{k}$ ) gives the following, which also generalises [20, Corollary 1.13].

Corollary 7.11. $\Gamma\left(Y, W_{Y / T}^{m}\right) \cong \bigoplus_{e \in E_{K}} z^{e} \cdot \wedge^{m}\left(\cap_{H \in \mathcal{F}_{\max } \backslash \mathcal{F}: e \in H}\left(H^{g p} \otimes \mathbb{k}\right) /\left(Q^{g p} \otimes \mathbb{K}\right)\right)$ with differential $d\left(z^{e} \cdot n\right)=z^{e} \cdot[e] \wedge n$.

With $c: Y \rightarrow A_{P, \mathcal{F}}$ the notation from before, we apply $c^{*}$ to the split exact sequence given by the top row of (7.1) and obtain another split exact sequence. The left term is free and $c^{*} W_{f}^{m}$ is reflexive by Corollary 7.9. Hence, $c^{*} W_{A_{P, \mathcal{F}} / \mathbb{k}}^{m}$ is also reflexive. With $V=c^{-1}(U)$, we find the natural surjection $c^{*} \Omega_{U / \mathbb{k}}^{\bullet} \rightarrow \Omega_{V / \mathbb{k}}^{\bullet}$ to be an isomorphism (e.g., by local freeness of both). For $j: V \hookrightarrow Y$ the inclusion and $W_{Y}^{\bullet}:=j_{*} \Omega_{V / \mathbb{k}}^{\bullet}$ we thus have $c^{*} W_{A_{P, \mathcal{F}} / \mathbb{k}}^{m} \cong W_{Y}^{m}$. Plugging this into Proposition 7.2 yields the following.

Corollary 7.12. $\Gamma\left(Y, W_{Y}^{m}\right) \cong \bigoplus_{e \in E_{K}} z^{e} \cdot \wedge^{m}\left(\bigcap_{H \in \mathcal{F}_{\max } \backslash \mathcal{F}: e \in H} H^{g p} \otimes \mathbb{k}\right)$ with differential $d\left(z^{e} \cdot n\right)=$ $z^{e} \cdot e \wedge n$.

\subsection{Local Analytic Theory}

We keep the setup and notation from before (with $\mathbb{k}=\mathbb{C}$ ), so $(Q \subset P, \mathcal{F})$ is an ETD and $K \subset Q$ a monoid ideal. We additionally assume that $Q \backslash K$ is finite, so $\mathcal{T}=\mathbb{C}[Q] /(K)$ is an Artinian local ring. For $P^{+}=P \backslash\{0\}$, let $\mathbb{C} \llbracket P \rrbracket$ be the completion of $\mathbb{C}[P]$ in $\left(P^{+}\right)$.

Lemma 7.13. ([39, Proposition V.1.1.3]). For every local homomorphism $h: P \rightarrow \mathbb{N}$; that is, $h^{-1}(0)=$ $\{0\}$ and we may view h as a grading; thus, it holds that

$$
\mathcal{O}_{A_{P}^{a n}, 0}=\left\{\sum_{p \in P} \alpha_{p} z^{p} \mid \alpha_{p} \in \mathbb{C}, \sup _{p \in P^{+}}\left\{\frac{\log \left|\alpha_{p}\right|}{h(p)}\right\}<\infty\right\} \subset \mathbb{C} \llbracket P \rrbracket .
$$

We have $\Gamma\left(Y, \mathcal{O}_{Y}\right) \cong \mathbb{C}\left[E_{K}\right]:=\bigoplus_{e \in E_{K}} \mathbb{C} \cdot z^{e}$ with $z^{e} \cdot z^{e^{\prime}}=z^{e+e^{\prime}}$ if $e+e^{\prime} \in E_{K}$ and $z^{e} \cdot z^{e^{\prime}}=0$ otherwise. By [23, Corollary 3.2] and Lemma 7.13, the complete local ring at the origin in $Y^{a n}$ is

$$
\hat{\mathcal{O}}_{Y, 0} \cong(\mathbb{C}[Q] /(K)) \otimes_{\mathbb{C} \llbracket Q \rrbracket} \mathbb{C} \llbracket P \rrbracket \cong\left\{\sum_{e \in E_{K}} \alpha_{e} z^{e}\right\}=: \mathbb{C} \llbracket E_{K} \rrbracket .
$$


Lemma 7.13 together with the surjectivity of $\mathcal{O}_{A_{P}^{a n}, 0} \rightarrow \mathcal{O}_{Y^{a n}, 0}$ and Krull's intersection theorem yields

$$
\mathcal{O}_{Y^{a n}, 0}=\left\{\sum_{e \in E_{K}} \alpha_{e} z^{e} \in \mathbb{C} \llbracket E_{K} \rrbracket \mid \sup _{e \in E_{K} \backslash 0}\left\{\frac{\log \left|\alpha_{e}\right|}{h(e)}\right\}<\infty\right\} .
$$

Lemma 7.14. Let $(V,\langle\cdot, \cdot\rangle)$ be a finite-dimensional $\mathbb{C}$-vector space with a Hermitian inner product. For every $e \in E_{K}$, let $V_{e} \subset V$ be vector subspaces so that

$$
\tilde{V}:=\bigoplus_{e \in E_{K}} z^{e} \cdot V_{e} \subset V\left[E_{K}\right]
$$

is a $\mathbb{C}\left[E_{K}\right]$-module. Assume moreover that $V_{e} \subset V$ depends only on the set $F(e):=\{H \subset P$ a facet $\mid Q \subset$ $H, e \in H\}$. Set $V \llbracket E_{K} \rrbracket:=\prod_{e \in E_{K}} z^{e} \cdot V_{e}$ and $\mathcal{V}^{a n}:=\tilde{V} \otimes_{\mathbb{C}\left[E_{K}\right]} \mathcal{O}_{Y}$ an. We find its stalk at the origin to be

$$
\mathcal{V}_{0}^{a n} \cong\left\{\sum_{e \in E_{K}} z^{e} \cdot v_{e} \in V \llbracket E_{K} \rrbracket \mid \sup _{e \in E_{K} \backslash 0}\left\{\frac{\log \left\|v_{e}\right\|}{h(e)}\right\}<\infty\right\} .
$$

Proof. The set of possible $F(e)$ is finite, so there is only a finite set of different $V_{e}$. Choosing orthonormal bases for all $V_{e}$ allows reducing the assertion to (7.6). We leave the technical details to the reader.

Remark 7.15. We can use Lemma 7.14 to compute the stalk at 0 of the analytification of $W_{Y / T}^{m}$ and $W_{Y}^{m}$ by using Corollary 7.11 and Corollary 7.12, respectively.

\section{Base Change of Differentials for Log Toroidal Families}

Definition $8.1(\mathrm{BC})$. We say that a generically $\log$ smooth morphism $f: X \rightarrow S$ satisfies the base change property if for every strict morphism $T \rightarrow S$ of Noetherian fs $\log$ schemes, $m \in \mathbb{Z}$ and $c$ the map given by the Cartesian diagram

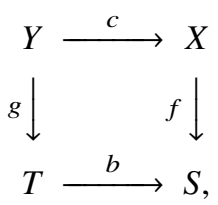

the sheaf $c^{*} W_{X / S}^{m}$ is reflexive or, equivalently, the natural map $c^{*} W_{X / S}^{m} \rightarrow W_{Y / T}^{m}$ is an isomorphism.

Theorem 8.2 (Base Change over Fields). Let $f: X \rightarrow S$ be a log toroidal family over a field $\mathbb{k} ;$ then $f$ satisfies $(B C)$.

Proof. This follows directly from the local statement Corollary 7.9.

Theorem 8.3 (Generic Base Change). Let $f: X \rightarrow S$ be a log toroidal family. Then there is a finite set of prime numbers $p_{1}, \ldots, p_{N} \in \mathbb{Z}$ so that if $f^{\circ}: X^{\circ} \rightarrow S^{\circ}$ is obtained from f by inverting $p_{1}, \ldots, p_{N}$ (i.e., base change to $\operatorname{Spec} \mathbb{Z}_{p_{1} \ldots p_{N}}$ ), then $f^{\circ}$ satisfies $(B C)$.

Proof. Again, this follows directly from the local statement Corollary 7.10 combined with the fact that we can use a finite cover by local models.

An application of the above theorems is the following lemma, which is crucial for the degeneration of the Hodge-de Rham spectral sequence. 
Lemma 8.4. (cf. [29, Proposition 6.6]). Let $f: X \rightarrow S$ be a proper $\log$ toroidal family with $S$ affine, and let $b: T \rightarrow S$ with T affine. Assume that $c^{*} W_{X / S}^{m}=W_{Y / T}^{m}$ holds for all $m$. Then we have isomorphisms

$$
\begin{aligned}
& L b^{*} R f_{*} W_{X / S}^{p} \rightarrow R g_{*} W_{Y / T}^{p} \\
& L b^{*} R f_{*} W_{X / S}^{\bullet} \rightarrow R g_{*} W_{Y / T}^{\bullet}
\end{aligned}
$$

in $D^{b}(T)$. If, for fixed $p$, all $R^{q} f_{*} W_{X / S}^{p}$ are locally free of constant rank, then (8.1) induces an isomorphism

$$
b^{*} R^{q} f_{*} W_{X / S}^{p} \stackrel{\cong}{\rightrightarrows} R^{q} g_{*} W_{Y / T}^{p} .
$$

If, for all $n$, the sheaf $R^{n} f_{*} W_{X / S}^{\bullet}$ is locally free of constant rank, then (8.2) induces an isomorphism

$$
b^{*} R^{n} f_{*} W_{X / S}^{\bullet} \stackrel{\cong}{\rightarrow} R^{n} g_{*} W_{X / S}^{\bullet}
$$

Proof. Because $W_{X / S}^{m}$ is flat over $S$ - this is Corollary 7.4 - the proof becomes identical to that in [29, Proposition 6.6].

\section{Spreading Out Log Toroidal Families}

We fix a sharp toric monoid $Q$, a field $\mathbb{k} \supset \mathbb{Q}$ and $\operatorname{set} S=\operatorname{Spec}(Q \rightarrow \mathbb{k})$ where the map $Q \rightarrow \mathbb{k}$ is $q \mapsto 0$ except $0 \mapsto 1$. We choose distinct subrings $B_{\lambda} \subset \mathbb{k}$ for all $\lambda$ in some index set $\Lambda$ so that any two $B_{\lambda_{1}}, B_{\lambda_{2}}$ are both contained in a third $B_{\lambda}$. We say $\lambda_{1} \leq \lambda_{2}$ if $B_{\lambda_{1}} \subset B_{\lambda_{2}}$. Furthermore, we require $\lim _{\lambda} B_{\lambda}=\mathbb{k}$ and that each $B_{\lambda}$ is of finite type over $\mathbb{Z}$. We get $\log$ schemes $S_{\lambda}=\operatorname{Spec}\left(Q \rightarrow B_{\lambda}\right)$ each with a strict map $S \rightarrow S_{\lambda}$ and, in fact, $S=\lim _{\lambda} S_{\lambda}$.

Proposition 9.1. Let $f: X \rightarrow S$ be a log toroidal family of relative dimension $d=\mathrm{rk} \Omega_{U / S}^{1}$. Then there is $\lambda \in \Lambda$ and a log toroidal family $f_{\lambda}: X_{\lambda} \rightarrow S_{\lambda}$, so that $f$ is obtained by base change from $f_{\lambda}$; that is, there is a Cartesian square

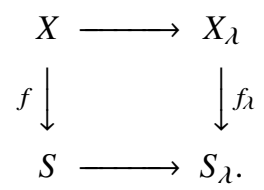

Iff is separated and/or proper, we can assume $f_{\lambda}$ to be so, too.

Proof. By [25, Theorem 8.8.2 (ii)], [25, Theorem 8.10.5] and [25, Theorem 11.2.6 (ii)] we can find a $\lambda \in \Lambda$ and a morphism $f_{\lambda}: X_{\lambda} \rightarrow S_{\lambda}$ that is finitely presented and flat and an isomorphism $S \times_{S_{\lambda}} X_{\lambda} \cong X$ over $S$. If $f: X \rightarrow S$ is separated respective proper, we can choose $f_{\lambda}$ moreover separated respective proper. Using [25, Corollaire 12.1.7(iii)] and [25, Theorem 8.10.5], we can choose $\lambda$ such that $f_{\lambda}$ is a Cohen-Macaulay morphism. Because these decompose disjointly over the relative codimension, again by increasing $\lambda$ if needed, we may assume that $f_{\lambda}$ has relative dimension $d$.

We next spread out $U$ such that $U_{\lambda} \subset X_{\lambda}$ satisfies (CC). We do this by spreading out its complement $Z$. Indeed, by [47, 05M5, Lemma 31.16.1], we can increase $\lambda$ so that every fibre of $Z_{\lambda} \rightarrow S_{\lambda}$ has dimension $\leq d-2$ and then define $U_{\lambda}:=X_{\lambda} \backslash Z_{\lambda}$.

Now a straightforward generalisation of the method of [48, Lemma 4.11.1] yields that, for appropriate $\lambda$, we can find a $\log$ structure on $U_{\lambda}$ and upgrade $f_{\lambda}$ to a $\log$ morphism such that $U_{\lambda}$ is fs and $f_{\lambda}$ is $\log$ smooth and saturated. More precisely, we choose an affine étale cover $\left\{U_{i}\right\}_{i}$ of $U$ such that $U_{i} \rightarrow S$ admits a local model by a saturated morphism $\theta_{i}: Q \rightarrow P_{i}$ of monoids; the local model remains a local 


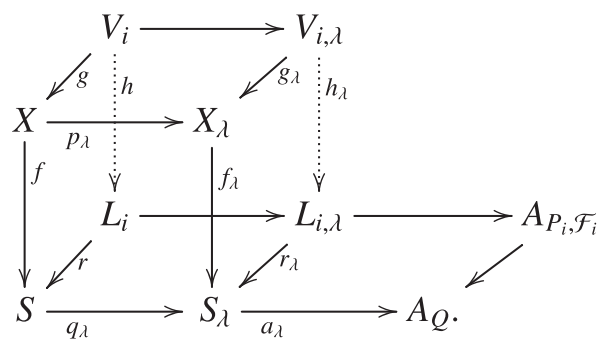

Figure 9.1. The diagram constructed in the text.

model for an appropriate spread-out $U_{i, \lambda} \rightarrow S_{\lambda}$ (for appropriate $\lambda$ ), which thus carries the structure of a saturated $\log$ smooth morphism to $S_{\lambda}$. At this point, the $U_{i, \lambda}$ might not cover $U_{\lambda}$, the $\log$ structures on $U_{i, \lambda}$ might not coincide on overlaps, and even if this was the case, we might not have a log morphism to $S_{\lambda}$. We achieve all of this by increasing $\lambda$.

Finally - again by possibly increasing $\lambda$ - we show that the family $f_{\lambda}: X_{\lambda} \rightarrow S_{\lambda}$ is log toroidal. We fix a finite covering $\left\{V_{i} \rightarrow X\right\}$ with local models $\left(Q \subset P_{i}, \mathcal{F}_{i}\right)$ as in Definition 4.1, and for each of them we construct a diagram as in Figure 9.1. Namely, we first spread out $V_{i} \rightarrow S$ to $V_{i, \lambda} \rightarrow S_{\lambda}$. Then $L_{i, \lambda}$ is defined by base change, and we construct the étale morphisms of schemes $g_{\lambda}: V_{i, \lambda} \rightarrow X_{\lambda}$ and $h_{\lambda}: V_{i, \lambda} \rightarrow L_{i, \lambda}$ also by spreading out. We can assume that $X_{\lambda}$ is covered by $\left\{V_{i, \lambda} \rightarrow X_{\lambda}\right\}$ and that $\tilde{U}_{i} \subset V_{i}$ spreads out to an open $\tilde{U}_{i, \lambda} \subset V_{i, \lambda}$ satisfying (CC). We get two log structures $\left(g_{\lambda}\right)_{\log }^{*} \mathcal{M}_{X_{\lambda}}$ and $\left(h_{\lambda}\right)_{\text {log }}^{*} \mathcal{M}_{L_{i, \lambda}}$ on $\tilde{U}_{i, \lambda}$, which we identify by [48, Sublemma 4.11.3]. By the same sublemma, the two morphisms $(g \circ f)_{l o g}^{*} \mathcal{M}_{S_{\lambda}} \rightarrow \mathcal{M}_{\tilde{U}_{i, \lambda}}$ coming from $f_{\lambda} \circ g_{\lambda}$ respectively $r_{\lambda} \circ h_{\lambda}$ coincide. Because $\left\{V_{i} \rightarrow X\right\}$ is a finite covering, we can find $\lambda$ that admits the above construction for all $V_{i}$ simultaneously.

\section{The Cartier Isomorphism}

In this section, we define the Cartier homomorphism for a generically log smooth family $f: X \rightarrow S$ in characteristic $p>0$. We then prove that it is an isomorphism if $f$ is $\log$ toroidal. Similar to [5], we first study the situation on $U$ and then examine its extension to all of $X$. Let $F_{S}: S \rightarrow S$ be the absolute $\log$ Frobenius on the base; that is, given by taking $p$ th power in $\mathcal{M}_{S}$ and $\mathcal{O}_{S}$ respectively, we similarly define $F_{X}: X \rightarrow X$. We define $f^{\prime}: X^{\prime} \rightarrow S$ and the relative Frobenius $F$ by the Cartesian square

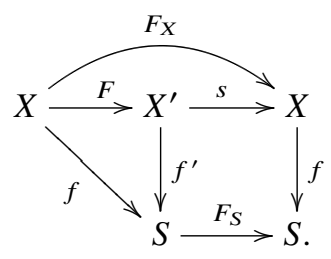

Set $U^{\prime}:=s^{-1}(U)$ and $Z^{\prime}=X^{\prime} \backslash U^{\prime}$.

Theorem 10.1 ([32]). We have a canonical (Cartier) isomorphism of $\mathcal{O}_{U^{\prime}}$-modules

$$
C_{U}^{-1}: \Omega_{U^{\prime} / S}^{m} \rightarrow \mathcal{H}^{m}\left(F_{*} \Omega_{U / S}^{\bullet}\right)
$$

which is compatible with $\wedge$ and satisfies $C^{-1}(a)=F^{*}(a)$ for $a \in \mathcal{O}_{X^{\prime}}$ and $C^{-1}\left(\operatorname{dlog}\left(s^{*} q\right)\right)=\operatorname{dlog}(q)$ for $q \in \mathcal{M}_{U}$.

Proof. This is [32, Theorem 4.12(1)] once we identify $U^{\prime \prime}=U^{\prime}$ : Kato considers the factorisation $U \stackrel{g}{\rightarrow} U^{\prime \prime} \stackrel{h}{\rightarrow}\left(U^{\prime}\right)^{\text {int }} \stackrel{i}{\rightarrow} U^{\prime}$ of $\left.F\right|_{U}$ where $i$ is the integralisation of $U^{\prime}$ and $g \circ h$ is the unique 
factorisation of this weakly purely inseparable morphism where $h$ is étale and $g$ purely inseparable, using [32, Proposition 4.10(2)]. Now $i$ is an isomorphism because $f$ is integral. By [39, Corollary III.2.5.4], because $f: U \rightarrow S$ is saturated, $F: U \rightarrow U^{\prime}$ is exact. The uniqueness of the factorisation $g \circ h$ now implies that $h$ is an isomorphism.

Because $W_{X^{\prime} / S}^{m}$ is $Z^{\prime}$-closed, pushing forward the inverse of $C_{U}^{-1}$ to $X^{\prime}$, we obtain a homomorphism

$$
C: \mathcal{H}^{m}\left(F_{*} W_{X / S}^{\bullet}\right) \rightarrow W_{X^{\prime} / S}^{m},
$$

which is an isomorphism on $U^{\prime}$. We obtain the following lemma.

Lemma 10.2. The map $C$ is an isomorphism if and only if $\mathcal{H}^{m}\left(F_{*} W_{X / S}^{\bullet}\right)$ is $Z^{\prime}$-closed.

Definition 10.3. We say that a generically $\log$ smooth family $f: X \rightarrow S$ in positive characteristic has the Cartier isomorphism property if $C$ is an isomorphism for all $m \geq 0$.

By Theorem 10.1, $\mathcal{H}^{m}\left(F_{*} W_{X / S}^{\bullet}\right)$ is locally free on $U^{\prime}$; hence, it is $Z^{\prime}$-closed if and only if it is reflexive. Reflexivity can be checked étale locally.

Lemma 10.4. Let $(Q \subset P, \mathcal{F})$ be an ETD, let $b: T \rightarrow A_{Q}$ be strict with $\underline{T}=\operatorname{Spec} \mathcal{T}$ and consider the Cartesian diagram

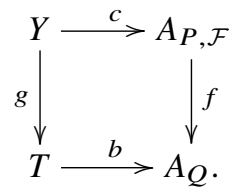

Then $\mathcal{H}^{m}\left(F_{*} W_{Y / T}^{\bullet}\right)$ is reflexive.

Corollary 10.5. Every log toroidal family $f: X \rightarrow S$ over $\mathbb{F}_{p}$ has the Cartier isomorphism property.

Proof of Lemma 10.4. Set $V:=c^{-1}\left(U_{P}\right)$ and let $Y^{\prime}, V^{\prime}$ be the base changes by the absolute Frobenius $F_{T}$. Let $F: Y \rightarrow Y^{\prime}$ be the relative Frobenius. Inspired by the Frobenius decomposition [13, Theorem 2.1], we construct a homomorphism $\phi^{\bullet}: \bigoplus_{m} W_{Y^{\prime} / T}^{m}[-m] \rightarrow F_{*} W_{Y / T}^{\bullet}$ of complexes of $\mathcal{O}_{Y^{\prime}-\text { modules }}$ that induces an isomorphism in cohomology. Because the left-hand side has zero differentials, the assertion then follows from the reflexivity of $W_{Y^{\prime} / T}^{m}$ given by Lemma 2.4.

Similar to Subsection 7.1, we find explicitly that $R^{\prime}:=\Gamma\left(Y^{\prime}, \mathcal{O}_{Y^{\prime}}\right)=\bigoplus_{e \in E} z^{e} \cdot \mathcal{T}$ with

$$
z^{e_{1}} \cdot z^{e_{2}}=z^{e} \cdot \sigma(q)^{p} \quad \text { whenever } \quad e_{1}+e_{2}=e+q
$$

with $e \in E, q \in Q$. We have $s^{*}\left(z^{e} \cdot t\right)=z^{e} \cdot t^{p}$ and $F^{*}\left(z^{e} \cdot t\right)=z^{p \cdot e} \cdot t$. After writing $W_{e}^{m}:=\left(W_{f}^{m}\right)_{e} \otimes_{\mathbb{F}_{p}} \mathcal{T}$, the module $\Gamma\left(Y^{\prime}, W_{Y^{\prime} / T}^{m}\right)$ is given by the $\mathcal{T}$-module $\bigoplus_{e \in E} z^{e} \cdot W_{e}^{m}$ on which $R^{\prime}$ acts as

$$
\left(z^{e_{1}} \cdot t_{1}\right) \cdot\left[z^{e_{2}} \cdot\left(w \otimes t_{2}\right)\right]=z^{e} \cdot\left(w \otimes \sigma(q)^{p} t_{1} t_{2}\right) \quad \text { whenever } \quad e_{1}+e_{2}=e+q
$$

with $e \in E, q \in Q$. Similarly, $\Gamma\left(Y^{\prime}, F_{*} W_{Y / T}^{m}\right)$ is given by the same $\mathcal{T}$-module, however now with $R^{\prime}$ acting via $F^{*}$ as

$$
\left(z^{e_{1}} \cdot t_{1}\right) \cdot\left[z^{e_{2}} \cdot\left(w \otimes t_{2}\right)\right]=z^{e} \cdot\left(w \otimes \sigma(q) t_{1} t_{2}\right) \quad \text { whenever } \quad p \cdot e_{1}+e_{2}=e+q .
$$

Note the subtle difference. The differential on $F_{*} W_{Y / T}^{\bullet}$ is given by $d\left(z^{e} \cdot(w \otimes t)\right)=z^{e} \cdot([e] \wedge w \otimes t)$. We define

$$
\phi^{\bullet}: \bigoplus_{m} W_{Y^{\prime} / T}^{m}[-m] \rightarrow F_{*} W_{Y / T}^{\bullet}, \quad z^{e} \cdot(w \otimes t) \mapsto z^{p \cdot e} \cdot(w \otimes t),
$$




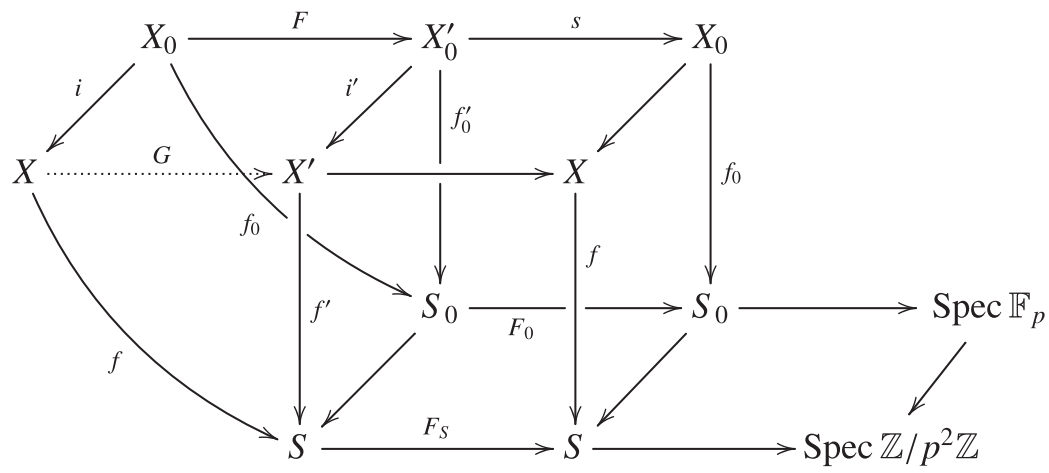

Figure 11.1. The diagram.

and claim that $\mathcal{H}^{m}\left(\phi^{\bullet}\right)$ is an isomorphism. Indeed, first note that $\phi^{\bullet}$ itself is injective. Then set $E_{p}=\{p \cdot e \mid e \in E\}$. We have $\operatorname{im}\left(\phi^{m}\right)=\bigoplus_{e \in E_{p}} z^{e} \cdot W_{e}^{m}$ because $W_{e}^{m}=W_{e / p}^{m}$ for $e \in E_{p}$ by Proposition 7.3. Denoting the coboundaries of $F_{*} W_{Y / T}^{m}$ by $B^{m}$, we have $\operatorname{im}\left(\phi^{m}\right) \cap B^{m}=0$ because $0=[e] \in W_{e}^{1}$ for $e \in E_{p}$ because $e=p e^{\prime}$ and $p$ is zero in $\mathcal{T}$. This readily gives that $\mathcal{H}^{m}\left(\phi^{\bullet}\right)$ is injective. For surjectivity, if $e \notin E_{p}$, observe that $[e] \neq 0$, so if $w \in W_{e}^{m}$, then $[e] \wedge w=0$ if and only if there is some $w^{\prime} \in W_{e}^{m-1}$ with $[e] \wedge w^{\prime}=w$.

Remark 10.6. We believe that $\mathcal{H}^{m}\left(\phi^{\bullet}\right)$ is the $\log$ Cartier isomorphism on $V^{\prime}$.

\section{The Decomposition of $F_{*} W_{X_{0} / S_{0}}^{\bullet}$}

We prove a log version of the decomposition theorem [13, Theorem 2.1] in the setting of generically log smooth families. (We noticed that [13, Corollary 3.7] alias [29] does not generalise well to the generically $\log$ smooth setting.) The assumption for $f: X \rightarrow S$ to be saturated on the log smooth locus allows a simpler approach than [32, Theorem 4.12]. Our setting is as follows: let $k$ be a perfect field with char $k=p$ (thus, $\mathbb{Z} / p^{2} \mathbb{Z} \rightarrow W_{2}(k)$ is flat), and let $Q$ be a sharp toric monoid. Set $S_{0}=\operatorname{Spec}(Q \rightarrow k)$ and $S=\operatorname{Spec}\left(Q \rightarrow W_{2}(k)\right)$ where in both cases $Q \ni q \mapsto 0$ except $0 \mapsto 1$. The Frobenius endomorphism on $k$ becomes an endomorphism $F_{0}$ of $S_{0}$ via $Q \ni q \mapsto p q$. Similarly, its lift to $W_{2}(k)$ defined via $\left(a_{1}, a_{2}\right) \mapsto\left(a_{1}^{p}, a_{2}^{p}\right)$ becomes $^{1}$ an endomorphism $F_{S}$ of $S$ that restricts to $F_{0}$ on $S_{0}$. Let $f: X \rightarrow S$ be a generically $\log$ smooth family and let $f_{0}: X_{0} \rightarrow S_{0}$ be its restriction to $S_{0}$. We consider the commutative diagram of generically $\log$ smooth families as in Figure 11.1, where $X_{0}^{\prime}, X^{\prime}$ are defined by requiring the front and back square to be Cartesian and $F$ is the relative Frobenius; that is, $F$ is induced by the back square's Cartesianness using the Frobenius endomorphisms on $X_{0}$ and $S_{0}$. Because $X$ does not have a Frobenius, we do not easily obtain the dotted arrow $G$ in a similar way and in general it does not exist globally. We call a locally defined morphism $G$ that fits into the diagram a local Frobenius lifting. Because the (Zariski or étale) topologies are identified along $F$ and $i$, we can define Frobenius liftings simply at the level of sheaves:

Definition 11.1. Let $Y^{\prime} \rightarrow X^{\prime}$ be an étale open. Then a Frobenius lifting $G: Y \rightarrow Y^{\prime}$ on $Y^{\prime}$ consists of a ring homomorphism $G^{*}: \mathcal{O}_{Y^{\prime}} \rightarrow G_{*} \mathcal{O}_{Y}$ yielding a morphism of schemes and a monoid homomorphism $G^{*}:\left.\left.\mathcal{M}_{Y^{\prime}}\right|_{V^{\prime}} \rightarrow G_{*} \mathcal{M}_{Y}\right|_{V^{\prime}}$ defined on some $V^{\prime} \subset Y^{\prime}$ satisfying $(C C)$, yielding a log morphism. Two Frobenius liftings are considered equal if they are equal on some smaller (Zariski) open satisfying $(C C)$. The Frobenius liftings form an étale sheaf of sets $\mathcal{F} r o b\left(X, X^{\prime}\right)$.

Remark 11.2. We need the flexibility of $V^{\prime}$ in the definition of $\mathcal{F r o b}\left(X, X^{\prime}\right)$ to construct Frobenius liftings from local models as they occur for log toroidal families. We will see below that we could have as well required the log part to be defined on $Y^{\prime} \cap U^{\prime}$; see the proof of Proposition 11.4.

${ }^{1}$ Warning: This is not the $p$ th power map on $W_{2}(k)$ and thus depends on the chosen chart. 
Let $j: U^{\prime} \hookrightarrow X^{\prime}$ denote the pullback of $U \subset X$ and $Z^{\prime}=X^{\prime} \backslash U^{\prime}$. By Lemma 2.3, Frob $\left(X, X^{\prime}\right)=$ $j_{*}\left(\left.\mathcal{F r o b}\left(X, X^{\prime}\right)\right|_{U^{\prime}}\right)$. Let $\mathcal{I} \subset \mathcal{O}_{X}$ be the ideal sheaf defining $X_{0} \subset X$; flatness gives $\mathcal{I}=p \cdot \mathcal{O}_{X} \cong \mathcal{O}_{X_{0}}$. Using $\mathcal{I}^{2}=0$, one checks that $F_{*} \mathcal{I}$ is an $\mathcal{O}_{X^{\prime}}$-module. Considering derivations on $U^{\prime}$ with values in $F_{*} \mathcal{I}$, we obtain a sheaf of groups $\mathcal{G}:=j_{*} \operatorname{Der}_{U^{\prime} / S}\left(F_{*} \mathcal{I}\right)=j_{*} \mathcal{H o m}\left(\Omega_{U^{\prime} / S}^{1}, F_{*} \mathcal{I}\right)$, which agrees with $\mathcal{H o m}\left(W_{X^{\prime} / S}^{1}, F_{*} \mathcal{I}\right)$ because $F_{*} \mathcal{I}$ is $Z^{\prime}$-closed by Lemma 2.3.

Lemma 11.3. The restriction $\left.\mathcal{F r o b}\left(X, X^{\prime}\right)\right|_{U^{\prime}}$ is a $\left.\mathcal{G}\right|_{U^{\prime} \text {-torsor; hence, } \mathcal{F} r o b}\left(X, X^{\prime}\right)$ is a $\mathcal{G}$-pseudotorsor.

Proof. Let $\mathcal{D}$ be the sheaf of sets on $U^{\prime}$ given by étale local deformations of the diagram

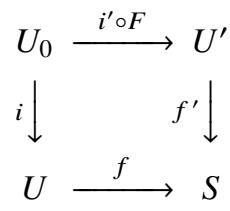

in the sense of [39, Definition IV.2.2.1]; that is, $\mathcal{D}$ is the sheaf of morphisms $U \rightarrow U^{\prime}$ making the diagram commute. The sheaf $\mathcal{D}$ is a $\left.\mathcal{G}\right|_{U^{\prime}}$-pseudo-torsor by [39, Theorem IV.2.2.2] and because $f^{\prime}: U^{\prime} \rightarrow S$ is smooth, it is a torsor. Because $\Omega_{U^{\prime} / S}^{1}$ is locally free, $\mathcal{D}$ is locally isomorphic to $\left(F_{*} \mathcal{I}\right)^{\oplus d}$. By Lemma 2.3, $\mathcal{D}$ is $\tilde{Z}$-closed for every $\tilde{Z} \subset X^{\prime}$ satisfying $\operatorname{codim}\left(\tilde{Z}, X^{\prime}\right) \geq 2$. By this property, the obvious homomorphism $\left.\mathcal{D} \rightarrow \mathcal{F r o b}\left(X, X^{\prime}\right)\right|_{U^{\prime}}$ is an isomorphism of sheaves of sets making $\left.\mathcal{F r o b}\left(X, X^{\prime}\right)\right|_{U^{\prime}}$ a $\left.\mathcal{G}\right|_{U^{\prime}}$-torsor.

Proposition 11.4. Let $Y^{\prime} \rightarrow X^{\prime}$ be an étale open and $G: Y \rightarrow Y^{\prime}$ a local Frobenius lifting. Then there is a canonical homomorphism of complexes

$$
\phi_{G}: W_{Y_{0}^{\prime} / S_{0}}^{1}[-1] \rightarrow F_{*} W_{Y_{0} / S_{0}}^{\bullet}
$$

inducing the Cartier isomorphism in first cohomology on $U_{0}^{\prime} \cap Y_{0}^{\prime}$. If $h \in \mathcal{G}\left(Y^{\prime}\right)$, then $\phi_{G}$ and $\phi_{h \cdot G}$ are related by

$$
\phi_{h \cdot G}=\phi_{G}+\left(F_{*} d\right) \circ \tilde{h}
$$

where $\tilde{h}: W_{Y_{0}^{\prime} / S_{0}}^{1} \rightarrow F_{*} \mathcal{I} \cong F_{*} W_{Y_{0} / S_{0}}^{0}$ is the induced homomorphism.

Proof. We choose $V^{\prime}=U^{\prime} \cap Y^{\prime}$ for the representative of $G$. The straightforward log version of the construction of [29, Proposition 3.8] yields a homomorphism $\Omega_{V_{0}^{\prime} / S_{0}}^{1} \rightarrow F_{*} \Omega_{V_{0} / S_{0}}^{1}$, and this has also been used implicitly by Kato in [32, Theorem 4.12]. Applying $j_{*}$ yields $\left(\phi_{G}\right)^{1}$, and we define the other $\left(\phi_{G}\right)^{m}$ to be 0 . The resulting $\phi_{G}$ does not depend on $V^{\prime}$ because the involved sheaves are $\tilde{Z}$-closed for every $\tilde{Z} \subset Y_{0}^{\prime}$ satisfying $\operatorname{codim}\left(\tilde{Z}, Y_{0}^{\prime}\right) \geq 2$, so $\phi_{G}$ is well defined. The construction yields that $\mathcal{H}^{1}\left(\phi_{G}\right)$ is the Cartier isomorphism of Theorem 10.1 on $V_{0}^{\prime}=U_{0}^{\prime} \cap Y_{0}^{\prime}$. The second statement is similar to [29, Lemma 5.4,(5.4.1)] except that we use the more elegant language of torsors (as already remarked in [13, Remark 2.2 (iii)]), which renders the analogue of [29, Lemma 5.4,(5.4.2)] trivial.

Theorem 11.5. Let $f: X \rightarrow S$ be a generically log smooth family, assume that $f_{0}: X_{0} \rightarrow S_{0}$ has the Cartier isomorphism property (Definition 10.3) and assume that $\mathcal{F} r o b\left(X, X^{\prime}\right)$ is a $\mathcal{G}$-torsor. Then we have a quasi-isomorphism

$$
\bigoplus_{m<p} W_{X_{0}^{\prime} / S_{0}}^{m}[-m] \rightarrow \tau_{<p} F_{*} W_{X_{0} / S_{0}}^{\bullet}
$$

in $D^{b}\left(X_{0}^{\prime}\right)$ where $\tau_{<p}$ means the truncation of a complex. 
Proof. Because $\mathcal{F r o b}\left(X, X^{\prime}\right)$ is a torsor, we can find an étale cover $\mathfrak{Y}=\left\{Y_{\alpha}^{\prime}\right\}$ of $X^{\prime}$ such that we have a local Frobenius lifting $G_{\alpha}: Y_{\alpha} \rightarrow Y_{\alpha}^{\prime}$. We obtain an induced cover $\mathfrak{Y}_{0}$ of $X_{0}^{\prime}$. On the log smooth locus $U_{0}^{\prime} \subset X_{0}^{\prime}$, we can apply an argument as implicitly used in [32, Theorem 4.12]: using Proposition 11.4, the gluing method of Step $\mathbf{B}$ in the proof of [29, Theorem 5.1] yields a homomorphism

$$
\varphi: \Omega_{U_{0}^{\prime} / S_{0}}^{1}[-1] \rightarrow \check{\mathcal{C}}^{\bullet}\left(\mathfrak{Y}_{0} \cap U_{0}^{\prime}, F_{*} \Omega_{U_{0} / S_{0}}^{\bullet}\right)=: \check{\mathcal{C}}_{U}^{\bullet}
$$

of complexes of sheaves where $\breve{\mathcal{C}}^{\bullet}\left(\mathfrak{U}, \mathcal{F}^{\bullet}\right)$ refers to the total sheaf Čech complex for a cover $\mathfrak{U}$ and a complex of sheaves $\mathcal{F}$. We also have the natural quasi-isomorphism

$$
\psi: F_{*} W_{X_{0} / S_{0}}^{\bullet} \rightarrow \check{\mathcal{C}}^{\bullet}\left(\mathfrak{Y}_{0}, F_{*} W_{X_{0} / S_{0}}^{\bullet}\right)
$$

Using $\psi$ and that the question is local, Proposition 11.4 gives that $\varphi$ induces the Cartier isomorphism on $U_{0}^{\prime}$ for $\mathcal{H}^{1}$. Now let $0 \leq m<p$. With the antisymmetrisation map $a_{m}: \Omega_{U_{0}^{\prime} / S_{0}}^{m}[-m] \rightarrow\left(\Omega_{U_{0}^{\prime} / S_{0}}^{1}[-1]\right)^{\otimes m}$ defined by $a_{m}\left(\omega_{1} \wedge \ldots \wedge \omega_{m}\right)=\frac{1}{m !} \sum_{\sigma \in S_{m}} \operatorname{sgn}(\sigma) \omega_{\sigma(1)} \otimes \ldots \otimes \omega_{\sigma(m)}$, we obtain a morphism

$$
\varphi^{m}: \Omega_{U_{0}^{\prime} / S_{0}}^{m}[-m] \stackrel{a_{m}}{\longrightarrow}\left(\Omega_{U_{0}^{\prime} / S_{0}}^{1}[-1]\right)^{\otimes m} \stackrel{\varphi^{\otimes m}}{\longrightarrow}\left(\check{\mathcal{C}}_{U}^{\bullet}\right)^{\otimes m} \rightarrow \check{\mathcal{C}}_{U}^{\bullet}
$$

where the last map is induced by the wedge product on $F_{*} \Omega_{U_{0} / S_{0}}^{\bullet}$. Note that the various $\varphi^{m}$ are compatible with the wedge product of $\Omega_{U_{0}^{\prime} / S_{0}}^{\bullet}$ and of the cohomology of $F_{*} \Omega_{U_{0} / S_{0}}^{\bullet}$; hence, $\varphi^{m}$ induces the Cartier isomorphism in cohomology. Taking the sum, we obtain a quasi-isomorphism

$$
\varphi^{\bullet}: \bigoplus_{m<p} \Omega_{U_{0}^{\prime} / S_{0}}^{m}[-m] \rightarrow \tau_{<p} \check{\mathcal{C}}_{U}^{\bullet} .
$$

Because $j_{*} \breve{\mathcal{C}}_{U}^{\bullet}=\check{\mathcal{C}}^{\bullet}\left(\mathfrak{Y}_{0}, F_{*} W_{X_{0} / S_{0}}^{\bullet}\right)$, we obtain the desired homomorphism in $D^{b}\left(X_{0}^{\prime}\right)$ as $\psi^{-1} \circ j_{*} \varphi^{\bullet}$. It is a quasi-isomorphism because $f_{0}: X_{0} \rightarrow S_{0}$ has the Cartier isomorphism property by assumption.

We like to apply this theorem to the case of a log toroidal family. It remains only to show that $\operatorname{Frob}\left(X, X^{\prime}\right)$ is a torsor.

Proposition 11.6. In the above situation, assume $f: X \rightarrow S$ log toroidal with respect to $S \rightarrow A_{Q}$. Then $\mathcal{F r o b}\left(X, X^{\prime}\right)$ is a $\mathcal{G}$-torsor; that is, Frobenius liftings exist locally.

Proof. Let $(Q \subset P, \mathcal{F})$ be an ETD from a local model of $f: X \rightarrow S$, as given in (LM) with $S=\tilde{S}$. Consider the diagram

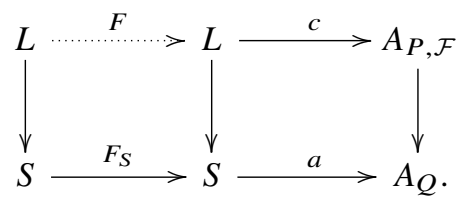

We claim that for the local existence of a Frobenius lifting, it suffices to show that there is a scheme morphism $F: \underline{L} \rightarrow \underline{L}$ that is the underlying morphism of a $\log$ morphism on $c^{-1}\left(U_{P}\right)$ such that the diagram commutes and the induced map $F \times_{S} S_{0}$ on $L_{0}=L \times_{S} S_{0}$ is the absolute Frobenius. Indeed, then $F$ plays the role of an absolute Frobenius on $L$, and its induced relative Frobenius gives rise to a local Frobenius lifting on $X^{\prime}$ via the local model.

The scheme $\underline{L}$ is affine with $\mathcal{O}(L)=\bigoplus_{e \in E} z^{e} \cdot W_{2}(k)$, allowing us to define $F: \underline{L} \rightarrow \underline{L}$ via $F^{*}\left(z^{e} \cdot w\right):=z^{\overline{p e}} \cdot F_{S}^{*}(w)$. It remains to extend $F$ to the $\log$ structure on $c^{-1}\left(U_{P}\right)$. Consider the maps of $\log$ schemes

$$
M:=\operatorname{Spec}(P \rightarrow \mathcal{O}(L)) \rightarrow L \rightarrow \operatorname{Spec}(Q \rightarrow \mathcal{O}(L))=: N
$$


With the notation of Corollary 3.11, we define $W_{i}:=c^{-1}\left(U_{i}\right)$. Observe that $\left.M\right|_{W_{1}}=\left.L\right|_{W_{1}}$ and $\left.L\right|_{W_{2}}=$ $\left.N\right|_{W_{2}}$. On $N$ and $M$, we get morphisms $F_{N}: N \rightarrow N$ and $F_{M}: M \rightarrow M$ by mapping $q \mapsto p \cdot q$ on the monoids and using $F^{*}$ on the rings. They are compatible with each other and with the maps to $S$; moreover, $F_{N} \times_{S} S_{0}$ and $F_{M} \times_{S} S_{0}$ are the absolute Frobenii on $N_{0}, M_{0}$. We define partially $\left.F\right|_{W_{1}}:=\left.F_{M}\right|_{W_{1}}$ and $\left.F\right|_{W_{2}}:=\left.F_{N}\right|_{W_{2}}$. Because $\left.N\right|_{W_{1} \cap W_{2}}=\left.L\right|_{W_{1} \cap W_{2}}=\left.M\right|_{W_{1} \cap W_{2}}$, these definitions agree on $W_{1} \cap W_{2}$ and we obtain a $\log$ morphism defined on $c^{-1}\left(U_{P}\right)=W_{1} \cup W_{2}$, which gives the desired map.

\section{The Hodge-de Rham Spectral Sequence}

We put the pieces together to prove Theorem 1.9 from the Introduction. Let $S=\operatorname{Spec}(Q \rightarrow \mathbb{k})$ for a field $\mathbb{k} \supset \mathbb{Q}$ with $Q \ni q \mapsto \delta_{q 0}$, and let $f: X \rightarrow S$ be a proper log toroidal family of relative dimension $d$ with respect to $S \rightarrow A_{Q}$. Setting $h^{p q}=\operatorname{dim}_{\mathbb{K}} R^{q} f_{*} W_{X / S}^{p}$ and $h^{n}=\operatorname{dim}_{\mathbb{K}} R^{n} f_{*} W_{X / S}^{\bullet}$, it suffices to prove $\sum_{p+q=n} h^{p q}=h^{n}$.

By Proposition 9.1, we can find an $S_{\lambda}=\operatorname{Spec}\left(Q \rightarrow B_{\lambda}\right)$ and a proper log toroidal family with respect to $S_{\lambda} \rightarrow A_{Q}$. Because $B_{\lambda}$ is integral, by shrinking $S_{\lambda}$, we can find a spreading out $\phi: \mathfrak{X} \rightarrow \mathcal{S}$ such that $R^{q} \phi_{*} W_{\mathfrak{X} / \mathcal{S}}^{p}$ and $R^{n} \phi_{*} W_{\mathfrak{X} / \mathcal{S}}^{\bullet}$ are locally free of constant rank $r^{p q}$ respectively $r^{n}$ and such that $\mathcal{S} / \mathbb{Z}$ is smooth as schemes. By Theorem 8.3 we can furthermore assume that $W_{\mathfrak{X} / \mathcal{S}}^{m}$ is compatible with any base change, and we can assume that $\operatorname{char} \kappa(s)>d$ for the residue field $\kappa(s)$ of every closed point $s \in \mathcal{S}$. Now let $\operatorname{Spec} k \rightarrow \mathcal{S}$ be a closed point. Because $\mathcal{S} / \mathbb{Z}$ is smooth, we can find a factorisation

$$
\operatorname{Spec} k \rightarrow \operatorname{Spec} W_{2}(k) \rightarrow \mathcal{S}
$$

that induces diagram (SO) from the introduction by strict base change. Setting $g^{p q}:=$ $\operatorname{dim}_{k} R^{q}\left(\phi_{k}\right)_{*} W_{\mathfrak{X}_{k} / k}^{p}$ and $g^{p q}:=\operatorname{dim}_{k} R^{n}\left(\phi_{k}\right)_{*} W_{\mathfrak{X}_{k} / k}$, Lemma 8.4 yields $h^{p q}=r^{p q}=g^{p q}$ and $h^{n}=r^{n}=g^{n}$; hence, it suffices to show $\sum_{p+q=n} g^{p q}=g^{n}$. Note that in diagram (SO) on the right, we are in the situation of Proposition 11.6, so by Theorem 11.5 we have a quasi-isomorphism

$$
\bigoplus_{m} W_{\mathfrak{X}_{k}^{\prime} / k}^{m}[-m] \simeq\left(F_{0}\right)_{*} W_{\mathfrak{X}_{k} / k}^{\bullet}
$$

Now a computation as in [13, Corollary 2.4] yields $\sum_{p+q=n} g^{p q}=g^{n}$, concluding the proof of Theorem 1.9.

\subsection{The Relative Spectral Sequence}

Proof of Theorem 1.10. It thereby suffices to show the surjectivity of

$$
\mathbb{H}^{k}\left(X, W_{X / S}^{\bullet}\right) \rightarrow \mathbb{H}^{k}\left(X_{0}, W_{X_{0} / S_{0}}^{\bullet}\right) .
$$

We prove this with the idea of [45, Section (2.6)] (cf. [34, Lemma 4.1] and [20, Theorem 4.1]). We define a complex

$$
\mathcal{L}^{\bullet}:=W_{X}^{\bullet, a n}[u]=\bigoplus_{s=0}^{\infty} W_{X}^{\bullet, a n} \cdot u^{s}, \quad d\left(\alpha_{s} u^{s}\right)=d \alpha_{s} \cdot u^{s}+s \delta(\rho) \wedge \alpha_{s} \cdot u^{s-1}
$$

of analytic sheaves where $\rho=f^{*}(1) \in \mathcal{M}_{X^{a n}}$ and $\delta: \mathcal{M}_{X^{a n}} \rightarrow W_{X}^{1, a n}$ is the log part of the universal derivation. Here $W_{X}^{\bullet}$,an denotes (the analytification of) absolute differentials as in Corollary 7.12. Projection to the $u^{0}$-summand composed with $W_{X}^{\bullet, a n} \rightarrow W_{X / S}^{\bullet \text {,an }}$ yields a map $\mathcal{L}^{\bullet} \rightarrow W_{X / S}^{\bullet \text {, an }}$ whose composition with $W_{X / S}^{\bullet, a n} \rightarrow W_{X_{0} / S_{0}}^{\bullet, a n}$ fits into an exact sequence

$$
0 \rightarrow \mathcal{K}^{\bullet} \rightarrow \mathcal{L}^{\bullet} \stackrel{\phi^{\bullet}}{\longrightarrow} W_{X_{0} / S_{0}}^{\bullet, a n} \rightarrow 0
$$


of complexes that defines $\mathcal{K}^{\bullet}$. Because $f: X \rightarrow S$ has ETD local models, we may use Corollaries 7.11, 7.12 and Remark 7.15 to have a local description of this sequence. Lemma 12.1 shows that $\mathcal{K}^{\bullet}$ is acyclic for all ETDs with 1-dimensional base, so $\phi^{\bullet}$ is a quasi-isomorphism and Theorem 1.10 follows by the discussion in Subsection 2.1.

Lemma 12.1. Let $(\mathbb{N} \subset P, \mathcal{F})$ be an ETD, and let $f: X \rightarrow S=S_{m}$ be the base change of $A_{P, \mathcal{F}} \rightarrow A_{\mathbb{N}}$ along $S_{m} \rightarrow A_{\mathbb{N}}$. With $0 \in A_{P, \mathcal{F}}$ denoting the origin, we have $\mathcal{H}^{k}\left(\mathcal{K}^{\bullet}\right)_{0}=0$ for all $k$.

Proof. We choose Hermitian inner products on the vector spaces $L:=P^{g p} \otimes \mathbb{C}$ and $W:=\left(P^{g p} \otimes\right.$ $\mathbb{C}) /\left(\mathbb{N}^{g p} \otimes \mathbb{C}\right)$. With $K=(m+1)+\mathbb{N} \subset \mathbb{N}$, we recall $E_{K}$ from Subsection 7.2. For $e \in E_{K}$, we define

$$
L_{e}:=\bigcap_{H \in \mathcal{F}_{\max } \backslash \mathcal{F}: e \in H} H^{g p} \otimes \mathbb{C} \quad \text { and } \quad W_{e}:=\bigcap_{H \in \mathcal{F}_{\max } \backslash \mathcal{F}: e \in H}\left(H^{g p} \otimes \mathbb{C}\right) /\left(\mathbb{N}^{g p} \otimes \mathbb{C}\right) .
$$

By Remark 7.15 and Lemma 7.14, elements of $\mathcal{L}_{0}^{k}$ are formal sums

$$
\left(\ell_{e, s}\right):=\sum_{s=0}^{N} \sum_{e \in E_{K}} u^{s} z^{e} \ell_{e, s}, \quad \ell_{e, s} \in \bigwedge^{k} L_{e}, \quad \sup _{\substack{e \in E_{K} \backslash 0 \\ 1 \leq s \leq N}}\left\{\log \left\|\ell_{e, s}\right\| / h(e)\right\}<\infty
$$

and elements of $W_{X_{0} / S_{0}, 0}^{k, a n}$ are formal sums

$$
\left(w_{e}\right):=\sum_{e \in E} z^{e} \cdot w_{e}, \quad w_{e} \in \bigwedge^{k} W_{e}, \quad \sup _{e \in E \backslash 0}\left\{\log \left\|w_{e}\right\| / h(e)\right\}<\infty .
$$

Note that $\left(\ell_{e, s}\right)$ is summed over $E_{K}$, whereas $\left(w_{e}\right)$ is summed over $E$. We denote the kernel of $\pi: \bigwedge^{k} L_{e} \rightarrow \bigwedge^{k} W_{e}$ by $K_{e}^{k}$ and observe $\phi\left(\left(\ell_{e, s}\right)\right)=\left(\pi\left(\ell_{e, 0}\right)\right)$, so $\left(\ell_{e, s}\right) \in \mathcal{K}_{0}^{k}$ if and only if $\ell_{e, 0} \in K_{e}^{k}$ for all $e \in E$. With $\bar{\rho}:=1 \otimes 1 \in \mathbb{N}^{g p} \otimes \mathbb{C}$, we have $\delta(\rho)=z^{0} \cdot \bar{\rho} \in W_{X}^{1}$, and thus

$$
d\left(\left(\ell_{e, s}\right)\right)=\left(e \wedge \ell_{e, s}+(s+1) \bar{\rho} \wedge \ell_{e, s+1}\right) .
$$

Let $\left(\ell_{e, s}\right) \in \mathcal{K}_{0}^{0}$ and assume $d\left(\left(\ell_{e, s}\right)\right)=0$. Because $\ell_{e, s} \in \mathbb{C}$, for $e \neq 0$ by descending induction in $s$ starting from $\ell_{e, N}$, we find $\ell_{e, s}=0$. We have $\ell_{0,0}=0$ and ascending induction yields $\ell_{0, s}=0$. Thus, $\mathcal{H}^{0}\left(\mathcal{K}^{\bullet}\right)_{0}=0$.

Next, let $\left(\ell_{e, s}\right) \in \mathcal{K}_{0}^{k+1}$ for $k \geq 0$ with $d\left(\left(\ell_{e, s}\right)\right)=0$. Starting with $e=0$, we construct $\left(\tau_{e, s}\right) \in \mathcal{K}_{0}^{k}$ with $d\left(\left(\tau_{e, s}\right)\right)=\left(\ell_{e, s}\right)$ using the following claim.

Claim 2. Let $(L,\langle\cdot, \cdot\rangle)$ be a $\mathbb{C}$-vector space of finite dimension with a Hermitian inner product. Let $0 \neq p \in L$ and $k \geq 0$, and assume $\ell \in \bigwedge^{k+1}$ L with $p \wedge \ell=0$. Then there is $a \tilde{\ell} \in \wedge^{k} L$ with $p \wedge \tilde{\ell}=\ell$ and $\|p\| \cdot\|\tilde{\ell}\|=\|\ell\|$.

Proof. Let $\ell_{1}:=\frac{p}{\|p\|}, \ell_{2}, \ldots, \ell_{n}$ be an orthonormal basis of $L$ and $\left\{\ell_{i_{1} \ldots i_{k}}\right\}$ the induced basis of $\wedge^{k} L$. If $\ell=\sum \alpha_{i_{1} \ldots i_{k+1}} \ell_{i_{1} \ldots i_{k+1}}$ satisfies the assumption, then $\tilde{\ell}=\frac{1}{\|p\|} \sum \alpha_{1 i_{2} \ldots i_{k+1}} \ell_{i_{2} \ldots i_{k+1}}$ is a solution.

We set $\tau_{0,0}=0$. Writing out (12.1) for $e=0$ yields

$$
d\left(\ell_{0,0}+\ell_{0,1} u+\ell_{0,2} u^{2}+\ldots\right)=\bar{\rho} \wedge \ell_{0,1}+2 \bar{\rho} \wedge \ell_{0,2} u+3 \bar{\rho} \wedge \ell_{0,3} u^{2}+\ldots
$$

and therefore $\bar{\rho} \wedge \ell_{0, i}=0$ for $i>0$. Because $\ell_{0,0} \in K_{0}^{0}$, we also have $\bar{\rho} \wedge \ell_{0,0}=0$. By Claim 2, there is $\tau_{0, s+1} \in \bigwedge^{k} L_{0}$ with $\bar{\rho} \wedge \tau_{0, s+1}=\ell_{0, s}$ and we are done with the case $e=0$. For $e \neq 0$ we need to care about convergence. Without loss of generality, $N \geq 1$. Because $e \wedge \ell_{e, N}=0$, we can find by Claim $2 \tau_{e, N} \in \wedge^{k} L_{e}$ with $e \wedge \tau_{e, N}=\ell_{e, N}$ and $\left\|\tau_{e, N}\right\| \cdot\|e\|=\left\|\ell_{e, N}\right\|$. For $s \geq 1$, we construct 
$\tau_{e, s} \in \bigwedge^{k} L_{e}$ by descending induction. Because of $e \wedge\left(\ell_{e, s}-(s+1) \bar{\rho} \wedge \tau_{e, s+1}\right)=0$, there is $\tau_{e, s}$ with $e \wedge \tau_{e, s}=\ell_{e, s}-(s+1) \bar{\rho} \wedge \tau_{e, s+1}$ and

$$
\left\|\tau_{e, s}\right\| \cdot\|e\|=\left\|\ell_{e, s}-(s+1) \bar{\rho} \wedge \tau_{e, s+1}\right\| .
$$

For $e \notin E$, we go one step further and construct $\tau_{e, 0} \in \bigwedge^{k} L_{e}$ with the same method, but for $e \in E$, the construction of $\tau_{e, 0} \in K_{e}^{k}$ is more intricate. We need another claim.

Claim 3. Let $(L,\langle\cdot, \cdot\rangle)$ be a $\mathbb{C}$-vector space of finite dimension with a Hermitian inner product. Let $0 \neq V, Y \subset L$ be subspaces with $V \cap Y=0$. Then there is a constant $\gamma>0$ with the following property: for every subspace $H$ with $V \subset H \subset L$ and $k \geq 0$, let $K_{H}^{k}$ be the kernel of $\wedge^{k} H \rightarrow \wedge^{k}(H / V)$. Then for every $0 \neq p \in Y \cap H$ and every $\ell \in K_{H}^{k+1}$ with $p \wedge \ell=0$, there is $a \tilde{\ell} \in K_{H}^{k}$ with $p \wedge \tilde{\ell}=\ell$ and $\gamma \cdot\|p\| \cdot\|\tilde{\ell}\| \leq\|\ell\|$.

Proof. Let $p=\left(p_{1}, p_{2}\right)$ be the decomposition of $p$ under $L=V \oplus V^{\perp}$, so $\|p\|^{2}=\left\|p_{1}\right\|^{2}+\left\|p_{2}\right\|^{2}$. Because $V \cap Y=0$, we have for $\gamma^{2}:=\inf _{0 \neq p \in Y}\left\|p_{2}\right\|^{2} /\|p\|^{2}$ that $0<\gamma \leq 1$. Let $\ell_{0}:=\frac{p_{2}}{\left\|p_{2}\right\|}, \ell_{1}, \ell_{2} \ldots$ be an orthonormal basis of $H$ and then $\bar{\ell}_{0}=\frac{p}{\|p\|}, \bar{\ell}_{i}:=\ell_{i}$ for $i>0$ is an ordinary basis of $H$. For $\ell=\sum \alpha_{i_{0} \ldots i_{k}} \bar{\ell}_{i_{0} \ldots i_{k}} \in K_{H}^{k+1}$ with $p \wedge \ell=0$, we define $\tilde{\ell}:=\frac{1}{\|p\|} \sum \alpha_{0 i_{1} \ldots i_{k}} \bar{\ell}_{i_{1} \ldots i_{k}} \in K_{H}^{k}$ to have $p \wedge \tilde{\ell}=\ell$. We also find

$$
\|\ell\|^{2}=\left\|\sum \alpha_{0 i_{1} \ldots i_{k}} \frac{p}{\|p\|} \wedge \ell_{i_{1} \ldots i_{k}}\right\|^{2} \geq\left\|\sum \alpha_{0 i_{1} \ldots i_{k}} \frac{p_{2}}{\|p\|} \wedge \ell_{i_{1} \ldots i_{k}}\right\|^{2} \geq \gamma^{2} \cdot\|p\|^{2} \cdot\|\tilde{\ell}\|^{2} .
$$

We apply Claim 3 to $L=P^{g p} \otimes \mathbb{C}$. Let $F_{e} \subset P$ be the face generated by $e$ and $Y=F_{e}^{g p} \otimes \mathbb{C}$. Let $V=\mathbb{N}^{g p} \otimes \mathbb{C}$ and $H=L_{e}$, so $K_{H}^{k}=K_{e}^{k}$. Then $e \wedge\left(\ell_{e, 0}-\bar{\rho} \wedge \tau_{e, 1}\right)=0$, so we find $\tau_{e, 0} \in K_{e}^{k}$ with $e \wedge \tau_{e, 0}=\ell_{e, 0}-\bar{\rho} \wedge \tau_{e, 1}$ and

$$
\gamma \cdot\left\|\tau_{e, 0}\right\| \cdot\|e\| \leq\left\|\ell_{e, 0}-\bar{\rho} \wedge \tau_{e, 1}\right\|
$$

The factor $\gamma$ depends on $Y$, but there are only finitely many faces generated by elements $e \in E$, so we take for $\gamma$ the minimum over them and furthermore $\gamma<1$. Applying the triangle inequality to the right-hand side of (12.3) and using induction and (12.2) yields

$$
\left\|\tau_{e, s}\right\| \leq \frac{1}{\gamma} \cdot \frac{1}{\|e\|} \sum_{k=s}^{N}\left(\frac{\|\bar{\rho}\|}{\|e\|}\right)^{k-s} \cdot \frac{k !}{s !} \cdot\left\|\ell_{e, k}\right\|
$$

for all $e \neq 0$. Because $\inf _{e \neq 0}\{\|e\|\}>0$, there is a bound $M>1$ independent of $e$ such that $\left\|\tau_{e, s}\right\| \leq$ $M \cdot \max _{k}\left\{\left\|\ell_{e, k}\right\|\right\}$, which proves

$$
\sup _{e \in E_{K} \backslash 0}\left\{\log \left\|\tau_{e, s}\right\| / h(e)\right\}<\infty
$$

and thus $\left(\tau_{e, s}\right) \in \mathcal{K}_{0}^{k}$. By construction, $d\left(\left(\tau_{e, s}\right)\right)=\left(\ell_{e, s}\right)$, so $\mathcal{H}^{k}\left(\mathcal{K}^{\bullet}\right)_{0}=0$.

\section{Smoothings via Maurer-Cartan Solutions}

In the upcoming Subsections 13.1 and 13.2, we adapt the methods of [7] to the setup given in the statement of Theorem 1.7. We then argue how to obtain an analytic smoothing from a formal one in Subsection 13.3. The combination of all of these sections gives a proof of Theorem 1.7. The main ingredients are Theorem 6.13, Theorem 1.9 and Theorem 1.10. A key ingredient is also Lemma 6.11 to know that $W_{X / S}^{d}$ is trivial for $d=\operatorname{dim} X$. 


\subsection{Constructing a Formal Deformation from a Solution to the Maurer-Cartan Equation}

We define ${ }^{k} S=\operatorname{Spec}\left(\mathbb{N} \stackrel{1 \mapsto t}{\longrightarrow} \mathbb{C}[t] / t^{k+1}\right)$ and assume we are given a proper log toroidal family ${ }^{0} X \rightarrow{ }^{0} S$. Let $\left\{{ }^{0} V_{\alpha}\right\}_{\alpha}$ be an affine cover of ${ }^{0} X$. For fixed $\alpha$, let $\left\{{ }^{k} V_{\alpha} \rightarrow{ }^{k} S\right\}_{k}$ be a system of deformations, compatible with restriction from $k$ to $k-1$ as obtained from Theorem 6.13 . Note that $V_{\alpha \beta}:={ }^{0} V_{\alpha} \cap{ }^{0} V_{\beta}$ is affine because ${ }^{0} X$ is separated. We give names to the restrictions of thickenings via ${ }^{k} V_{\alpha ; \alpha \beta}:=\left.{ }^{k} V_{\alpha}\right|_{V_{\alpha \beta}}$. Again by Theorem 6.13, we find isomorphisms

$$
{ }^{k} \phi_{\alpha \beta}:{ }^{k} V_{\alpha ; \alpha \beta} \rightarrow{ }^{k} V_{\beta ; \alpha \beta}
$$

of generically log smooth families over ${ }^{k} S$ that are compatible with the restrictions to the base changes via ${ }^{k-1} S \rightarrow{ }^{k} S$ but do not necessarily satisfy a cocycle condition.

We now analytify ${ }^{k} X \rightarrow{ }^{k} S$ as well as ${ }^{k} V_{\alpha},{ }^{k} V_{\alpha ; \alpha \beta}$. We keep using the same symbols though now refer to the analytifications respectively.

Let $\left\{U_{i}\right\}_{i \in I}$ be a cover of ${ }^{0} X$ by Stein open sets that is also a basis for the analytic topology of ${ }^{0} X$ with $I$ countable and totally ordered. Set $U_{i_{0} \ldots i_{l}}:=\bigcap_{k=0}^{l} U_{i_{k}}$. We obtain the sheaves of Gerstenhaber algebras

$$
{ }^{k} \mathcal{G}_{\alpha}^{p}:=\Theta_{\left({ }^{k} V_{\alpha}\right) / k}^{-p}
$$

concentrated in nonpositive degrees via the negative Schouten-Nijenhuis bracket $-[\cdot, \cdot]$ and $\wedge$. Set $\mathbf{\Delta}_{l}=\operatorname{Spec}\left(\mathbb{C}\left[x_{0}, \ldots, x_{n}\right] /\left(x_{0}+\cdots+x_{n}-1\right)\right)$ and $\mathcal{A}^{q}\left(\mathbf{\Delta}_{l}\right)=\Omega_{\mathbf{\Delta}_{l}}^{q}$ and let $d_{j, l}: \boldsymbol{\Delta}_{l-1} \rightarrow \boldsymbol{\Delta}_{l}$ be given by $x_{j} \mapsto 0$. One constructs the Thom-Whitney bicomplex

$$
{ }^{k} T W_{\alpha ; \alpha_{0} \ldots \alpha_{l}}^{p, q}=\left\{\begin{array}{l|l}
\left(\varphi_{i_{0} \ldots i_{l}}\right)_{i_{0}<\cdots<i_{l}} & \begin{array}{c}
U_{i_{j}} \subset V_{\alpha_{0}} \cap \ldots \cap V_{\alpha_{l}} \text { for } 0 \leq j \leq l, \\
\varphi_{i_{0} \ldots i_{l}} \in \mathcal{A}^{q}\left(\mathbf{\Lambda}_{l}\right) \otimes_{\mathbb{C}}{ }^{k} \mathcal{G}_{\alpha}^{p}\left(U_{i_{0} \ldots i_{l}}\right), \\
d_{j, l}^{*}\left(\varphi_{i_{0} \ldots i_{l}}\right)=\left.\varphi_{i_{0} \ldots \hat{i}_{j} \ldots i_{l}}\right|_{i_{i_{0}} \ldots i_{l}}
\end{array}
\end{array}\right\}
$$

The differential for the index $p$ is trivial and the differential $\bar{\partial}_{\alpha}$ for the index $q$ is induced by the de Rham differential on $\mathcal{A}^{q}\left(\boldsymbol{\Lambda}_{l}\right)$. Furthermore, $-[\cdot, \cdot]$ and $\wedge$ turn $T W$ into a Gerstenhaber algebra. For $W \subset V_{\alpha}$, let $\left.{ }^{k} T W_{\alpha ; \alpha}^{p, q}\right|_{W}$ be given by (TW) but with the additional requirement to have $U_{i_{j}} \subset W$. The presheaf $\left.W \mapsto{ }^{k} T W_{\alpha ; \alpha}^{p, \bullet}\right|_{W}$ gives a resolution of the sheaf ${ }^{k} \mathcal{G}_{\alpha}^{p}$ on $V_{\alpha}$, so ${ }^{k} \mathcal{G}_{\alpha}^{p}(W)=H_{\bar{\partial}_{\alpha}}^{0}\left(\left.{ }^{k} T W_{\alpha ; \alpha}^{p, \bullet}\right|_{W}\right)$.

The isomorphisms ${ }^{k} \phi_{\alpha \beta}$ induce isomorphisms ${ }^{k} \psi_{\alpha \beta}:\left.\left.{ }^{k} \mathcal{G}_{\alpha}^{\bullet}\right|_{V_{\alpha \beta}} \rightarrow{ }^{k} \mathcal{G}_{\beta}^{\bullet}\right|_{V_{\alpha \beta}}$ of sheaves of Gerstenhaber algebras, which can be used ([7, Key Lemma 3.21]) to construct isomorphisms

$$
{ }^{k} g_{\alpha \beta}:{ }^{k} T W_{\alpha ; \alpha \beta}^{p, q} \rightarrow{ }^{k} T W_{\beta ; \alpha \beta}^{p, q}
$$

that satisfy the cocycle condition ${ }^{k} g_{\gamma \alpha}{ }^{k} g_{\beta \gamma}{ }^{k} g_{\alpha \beta}=\mathrm{id}$ and are compatible with restriction from $k$ to $k-1$ and with $-[\cdot, \cdot]$ and $\wedge$. The cocycle condition allows one to glue $\left\{{ }^{k} T W_{\alpha}^{p, q}\right\}_{\alpha}$ to a presheaf ${ }^{k} \mathrm{PV}^{p, q}$ on ${ }^{0} X$ compatible with restricting from $k$ to $k-1$. We set ${ }^{k} \mathrm{PV}^{n}:=\bigoplus_{p+q=n}{ }^{k} \mathrm{PV}^{p, q}$.

Though ${ }^{k} g_{\alpha \beta}$ are not necessarily compatible with the differentials $\bar{\partial}_{\alpha}, \bar{\partial}_{\beta}$, there exist ${ }^{k} \mathfrak{D}_{\alpha} \in{ }^{k} T W_{\alpha}^{-1,1}$ such that $\left(\bar{\partial}_{\alpha}+\left[{ }^{k} \mathfrak{D}_{\alpha}, \cdot\right]\right)_{\alpha}$ gives a system of maps compatible with ${ }^{k} g_{\alpha \beta}$ ([7, Theorem 3.34]). This system glues to an operator $\bar{\partial}$ on ${ }^{k} \mathrm{PV}^{p, q}$ compatible with restriction from $k$ to $k-1$. However, $\bar{\partial}$ is not a differential because

$$
\bar{\partial}^{2}=\left[{ }^{k} \mathfrak{l}_{\alpha}, \cdot\right] \quad \text { for } \quad{ }^{k} \mathfrak{l}_{\alpha}:=\bar{\partial}_{\alpha}\left({ }^{k} \mathfrak{D}_{\alpha}\right)+\frac{1}{2}\left[{ }^{k} \mathfrak{D}_{\alpha},{ }^{k} \mathfrak{D}_{\alpha}\right] \in{ }^{k} T W_{\alpha}^{-1,2}
$$

The $\left\{{ }^{k} \mathfrak{I}_{\alpha}\right\}_{\alpha}$ glue to a global element ${ }^{k} \mathfrak{I} \in{ }^{k} \mathrm{PV}^{-1,2}$ that is compatible with restricting from $k$ to $k-1$. If ${ }^{k} \phi \in{ }^{k} \mathrm{PV}^{-1,1}$ solves the Maurer-Cartan equation

$$
\bar{\partial}\left({ }^{k} \phi\right)+\frac{1}{2}\left[{ }^{k} \phi,{ }^{k} \phi\right]+{ }^{k} \mathfrak{l}=0,
$$


then $\left(\bar{\partial}+\left[{ }^{k} \phi, \cdot\right]\right)^{2}=0$. In this case, the cohomology $H_{\left(\bar{\partial}+\left[{ }^{k} \phi, \cdot\right]\right)}\left({ }^{k} \mathrm{PV} \bullet\right)$ is a presheaf of Gerstenhaber algebras on ${ }^{0} X$ that is locally isomorphic to ${ }^{k} \mathcal{G}_{\alpha}^{\bullet}$. The sheafification of its degree 0 part gives a sheaf $\mathcal{O}_{X_{k}}$ of $\mathbb{C}[t] / t^{k+1}$-algebras on ${ }^{0} X$, which we take as the $k$ th-order deformation of ${ }^{0} X$. Taking the limit $\mathcal{O}_{\mathfrak{X}}:=\lim _{\longleftarrow} \mathcal{O}_{X_{k}}$ yields a flat and proper morphism $\mathfrak{X} \rightarrow \mathfrak{S}$ with $\mathfrak{S}:=\operatorname{Spf}(\mathbb{C} \llbracket t \rrbracket)$.

\subsection{Constructing a Solution to the Maurer-Cartan Equation Using the Batalin-Vilkovisky Operator}

We assume that $W_{{ }^{0} X /{ }^{0} S}^{d} \cong \mathcal{O}^{0_{X}}$. We fix a global generator ${ }^{0} \omega \in \Gamma\left({ }^{0} X, W^{d}{ }^{d}{ }^{k} S\right.$. Let ${ }^{k} \omega_{\alpha} \in$ $\Gamma\left({ }^{0} V_{\alpha}, W_{k_{V_{\alpha} /{ }^{k} S}}^{d}\right)$ be a choice of generator that is a lift to $k$ of $\left.{ }^{0} \omega\right|_{0} V_{\alpha}$. The Batalin-Vilkovisky operator ${ }^{k} \Delta_{\alpha}$ is the transfer of the de Rham differential $d$ to the polyvector fields; that is, ${ }^{k} \Delta_{\alpha}$ is the composition

$$
\Theta_{\left({ }^{k} V_{\alpha}\right) /{ }^{k} S}^{p} \stackrel{\left\llcorner\left({ }^{k} \omega_{\alpha}\right)\right.}{\longrightarrow} W_{\left({ }^{k} V_{\alpha}\right) /{ }^{k} S}^{d-p} \stackrel{\mathrm{d}}{\longrightarrow} W_{\left({ }^{k} V_{\alpha}\right) /{ }^{k} S}^{d-p+1} \stackrel{\left\llcorner\left({ }^{k} \omega_{\alpha}\right)^{-1}\right.}{\longrightarrow} \Theta_{\left({ }^{k} V_{\alpha}\right) /{ }^{k} S}^{p-1}
$$

and thus a differential ${ }^{k} \mathcal{G}_{\alpha}^{p} \rightarrow{ }^{k} \mathcal{G}_{\alpha}^{p+1}$. Choosing ${ }^{k} \omega_{\alpha}$ compatible with restricting from $k$ to $k-1$, the ${ }^{k} \Delta_{\alpha}$ also share this property. For $W \subset{ }^{0} V_{\alpha} \cap{ }^{0} V_{\beta}$ there is $\lambda_{\alpha \beta} \in \Gamma\left(W,{ }^{k} \mathcal{G}_{\alpha}^{0}\right)$ with $\left.{ }^{k} \omega_{\alpha}\right|_{W}=\left.\lambda_{\alpha \beta} \cdot{ }^{k} \omega_{\beta}\right|_{W}$. Setting ${ }^{k} \mathfrak{w}_{\alpha \beta}:=\log \left(\lambda_{\alpha \beta}\right)$ yields

$$
{ }^{k} \psi_{\beta \alpha} \circ{ }^{k} \Delta_{\beta} \circ{ }^{k} \psi_{\alpha \beta}-{ }^{k} \Delta_{\alpha}=\left[{ }^{k} \mathfrak{w}_{\alpha \beta}, \cdot\right],
$$

and then $\left\{{ }^{k} \mathfrak{w}_{\alpha \beta}\right\}_{\alpha \beta}$ can be upgraded ([7, Theorem 3.34]) to a Čech cocycle for ${ }^{k} T W_{\alpha ; \alpha \beta}^{0,0}$ that by exactness lifts to a collection ${ }^{k} \mathfrak{f}_{\alpha} \in T W_{\alpha}^{0,0}$. The collection is compatible with restricting from $k$ to $k-1$ and satisfies

$$
{ }^{k} g_{\beta \alpha} \circ\left({ }^{k} \Delta_{\beta}+\left[{ }^{k} \mathfrak{f}_{\beta}, \cdot\right]\right) \circ{ }^{k} g_{\alpha \beta}=\left({ }^{k} \Delta_{\alpha}+\left[{ }^{k} \mathfrak{f}_{\alpha}, \cdot\right]\right) .
$$

Because ${ }^{k} \mathfrak{f}_{\alpha}$ lives in degree $(0,0)$, one has $\left({ }^{k} \Delta_{\alpha}+\left[{ }^{k} \mathfrak{f}_{\alpha}, \cdot\right]\right)^{2}=0$, so we can glue the collection $\left\{{ }^{k} \Delta_{\alpha}+\left[{ }^{k} \mathfrak{f}_{\alpha}, \cdot\right]\right\}_{\alpha}$ to an operator $\Delta:{ }^{k} \mathrm{PV}^{p, q} \rightarrow{ }^{k} \mathrm{PV}^{p+1, q}$ with $\Delta^{2}=0$. Now,

$$
\Delta \bar{\partial}+\bar{\partial} \Delta=\left[{ }^{k} \mathfrak{y}, \cdot\right] \quad \text { for } \quad{ }^{k} \mathfrak{y}_{\alpha}:={ }^{k} \Delta_{\alpha}\left({ }^{k} \mathfrak{D}_{\alpha}\right)+{ }^{k} \bar{\partial}_{\alpha}\left({ }^{k} \mathfrak{f}_{\alpha}\right)+\left[{ }^{k} \mathfrak{D}_{\alpha},{ }^{k} \mathfrak{f}_{\alpha}\right]
$$

and ${ }^{k} \mathfrak{y} \in{ }^{k} \mathrm{PV}^{0,1}$ is glued from the collection ${ }^{k} \mathfrak{y}_{\alpha}$. By construction,

$$
\breve{d}:=\bar{\partial}+\Delta+(\mathfrak{l}+\mathfrak{y}) \wedge
$$

satisfies $\breve{d}^{2}=0$ and, furthermore, $(\mathfrak{l}+\mathfrak{y}) \equiv 0 \bmod (t)$.

Theorem 13.1. The natural maps $H_{\breve{d}}^{i}\left({ }^{k} \mathrm{PV} \bullet\right) \rightarrow H_{\breve{d}}^{i}\left({ }^{k-1} \mathrm{PV} \bullet\right)$ are surjective for all $i$ and $k$.

Proof. As in [7, Proposition 4.8], the elements $\exp \left({ }^{k} \mathfrak{f}_{\alpha}\llcorner){ }^{k} \omega_{\alpha}\right.$ glue to a global element ${ }^{k} \omega$ in the ThomWhitney de Rham complex $\left({ }_{\|}^{k} \mathcal{A}^{\bullet}, d\right)$ (constructed from $W_{k_{V_{\alpha}} /{ }^{k} S}$ in our case) compatible with restricting from $k$ to $k-1$. Contracting ${ }^{k} \omega$ gives an isomorphism of complexes ${ }^{k} \mathrm{PV}^{\bullet} \rightarrow{ }_{\|}^{k} \mathcal{A}^{\bullet}$, so it suffices to prove surjectivity of $H_{d}^{i}\left({ }_{\|}^{k} \mathcal{A}^{\bullet}\right) \rightarrow H_{d}^{i}\left({ }_{\|}^{k-1} \mathcal{A}^{\bullet}\right)$. This follows from Theorem 1.10 (cf. [7, Lemma 4.17]).

Remark 13.2. For a formal variable $u^{\frac{1}{2}}$, consider on $\mathrm{PV}^{\bullet} \llbracket u^{\frac{1}{2}} \rrbracket$ the differential $\breve{d}_{u}:=\bar{\partial}+u \Delta+u^{-1}(\mathfrak{I}+u \mathfrak{y}) \wedge$. A direct computation gives $\breve{d}_{u}=u^{\frac{1}{2}} I_{u}^{-1} \circ \breve{d} \circ I_{u}$ where $I_{u}$ is defined by $I_{u}(\varphi)=u^{\frac{p-q-2}{2}} \varphi$ for $\varphi \in$ $\mathrm{PV}^{p, q} \llbracket u^{\frac{1}{2}} \rrbracket\left[u^{-\frac{1}{2}}\right]$ (cf. [7, Notation 5.1]). Theorem 13.1 thus implies that

$$
H_{\breve{d}_{u}}^{i}\left({ }^{k} \mathrm{PV} \bullet \llbracket u^{\frac{1}{2}} \rrbracket\left[u^{-\frac{1}{2}}\right]\right) \rightarrow H_{\breve{d}_{u}}^{i}\left({ }^{k-1} \mathrm{PV} \bullet \llbracket u^{\frac{1}{2}} \rrbracket\left[u^{-\frac{1}{2}}\right]\right)
$$

is surjective for all $i, k$. 
Theorem 13.3. For all $i, H_{\bar{\partial}+u \Delta}^{i}\left({ }^{0} \mathrm{PV} \llbracket u \rrbracket\right)$ is a free $\mathbb{C} \llbracket u \rrbracket$-module of finite rank.

Proof. Note that $k=0$. With $\bar{\partial}$ the Čech differential for the cover $\left\{V_{\alpha}\right\}_{\alpha}$, the degeneration of the Hodge-de Rham spectral sequence for $\left(W_{0_{X /{ }^{S} S}}^{\bullet}, d\right)$ at $E_{1}$ by Theorem 1.9 is equivalent to $H_{\bar{\partial}+u d}^{i}\left(\left\{V_{\alpha}\right\}_{\alpha}, W_{0_{X} /{ }^{\circ} S}^{\bullet} \llbracket u \rrbracket\right)$ being a free $\mathbb{C} \llbracket u \rrbracket$-module of finite rank. The quasi-isomorphisms $W_{{ }^{0} X /{ }^{\circ} S} \llbracket u \rrbracket \rightarrow{ }_{\|}^{0} \mathcal{A}^{\bullet} \llbracket u \rrbracket$ and ${ }^{0} \mathrm{PV} \bullet \llbracket u \rrbracket \rightarrow{ }^{0} \mathcal{A}^{\bullet} \llbracket u \rrbracket$ yield the assertion.

Theorem 13.4. There exist ${ }^{k} \varphi \in{ }^{k} \mathrm{PV}^{0} \llbracket u \rrbracket$ for all $k \geq 0$ with ${ }^{k} \varphi \equiv{ }^{k+1} \varphi \bmod t^{k+1}$ and ${ }^{0} \varphi=0$ solving

$$
(\bar{\partial}+u \Delta)\left({ }^{k} \varphi\right)+\frac{1}{2}\left[{ }^{k} \varphi,{ }^{k} \varphi\right]+\left({ }^{k} \mathfrak{l}+u^{k} \mathfrak{y}\right)=0 .
$$

Furthermore, setting ${ }^{k} \phi:=\left({ }^{k} \varphi \bmod u\right)$ with ${ }^{k} \phi=\sum_{j}{ }^{k} \phi_{j}$ and ${ }^{k} \phi_{j} \in{ }^{k} \mathrm{PV}^{-j, j}$, it holds that ${ }^{k} \phi_{0}=0$ and thus ${ }^{k} \phi_{1} \in{ }^{k} \mathrm{PV}^{-1,1}$ solves (MC1).

Proof. The first assertion becomes [7, Theorem 5.5] if we set $\mathbf{I}=(t)$ and $\psi=0$ and check that we have the ingredients for its proof available. The proof goes by induction over $k$ and uses (i) the surjectivity in Theorem 13.1 for $k=0$, (ii) the surjectivity in Equation (13.1) for all $k$ and (iii) Theorem 13.3 in each step to get rid of negative powers of $u$ in ${ }^{k} \varphi$. The second statement is [7, Lemma 5.11].

\subsection{From a Formal Deformation to an Analytic Deformation}

Let $\subseteq$ be the completion of an analytic variety $S$ in a nonzero divisor $t \in \Gamma\left(S, \mathcal{O}_{S}\right)$. Let $S_{k}$ be the closed analytic subvariety defined by $t^{k}$. If $X \rightarrow S$ is flat, we denote by $X_{k} \rightarrow S_{k}$ the base change to $S_{k}$, similarly for a flat map $\mathfrak{X} \rightarrow \mathfrak{G}$.

Theorem 13.5 ([43], Theorem B.1). Given a proper and flat formal analytic morphism $\hat{\varphi}: \mathfrak{X} \rightarrow \mathfrak{G}$, for every $k>0$ there is a proper flat analytic morphism $\varphi: X \rightarrow S$ together with an $S_{k}$-isomorphism $\mathfrak{X}_{k} \rightarrow X_{k}$ of the base changes of $\hat{\varphi}$ and $\varphi$ to $S_{k}$.

Theorem 13.6 ([42], Theorem 5.5 (1)). In the situation of Theorem 13.5, given $s \in S_{0}$ and $X_{s}=\varphi^{-1}(s)$, there exists an integer $K>0$ such that whenever $\varphi: X \rightarrow S$ is obtained for $k>K$, every point $x \in X_{S}$ has a neighbourhood in $X$ whose t-completion is formally isomorphic to a neighbourhood of $x$ in $\mathfrak{X}$, in particular, if $\mathfrak{X}$ is a smoothing of a fibre $X_{s}$ for $t \neq 0$, then so is $X$.

Theorem 13.7 ([42], Theorem 5.5 (3)). In the situation of Theorem 13.6, for $X_{0}$ the base change to $S_{0}$, the maps of pairs $\left(X, X_{0}\right) \rightarrow\left(S, S_{0}\right)$ and $\left(\mathfrak{X}, \mathfrak{X}_{0}\right) \rightarrow\left(\mathfrak{S}, S_{0}\right)$ turn $\hat{\varphi}$ and $\varphi$ into log morphisms via the divisorial log structures. There is an isomorphism of the log fibres over $s \in S$ whose underlying morphism is the restriction to the fibre $X_{s}$ of the $S_{k}$-isomorphism $\mathfrak{X}_{k} \rightarrow X_{k}$.

Acknowledgements. The last author feels indebted to Arthur Ogus for almost a decade of communication on the challenges in proving Theorem 1.9. We thank Mark Gross for connecting the authors with K. Chan, C. Leung and Z.N. Ma, whom we also thank for supportive communication and harmonisation of our projects. We thank Hélène Esnault and Bernd Siebert for valuable communication and Stefan Müller-Stach for bringing the first and last authors together. Our gratitude for hospitality goes to JGU Mainz and the last author also thanks IAS Princeton and Univ. Hamburg. All authors were funded by DFG research grant RU 1629/4-1. S. Felten received a Doktorandenstipendium from the Carl Zeiss Foundation.

Conflict of Interest: None.

\section{References}

[1] M. Abouzaid, D. Auroux and L. Katzarkov, 'Lagrangian fibrations on blowups of toric varieties and mirror symmetry for hypersurfaces', Publ. Math. Inst. Hautes Études Sci. 123 (2016), 199-282.

[2] C. Bănică and O. Stănăşilă, Algebraic Methods in the Global Theory of Complex Spaces (Bucharest; John Wiley \& Sons, London, 1976). Translated from Romanian. 
[3] S. Barannikov and M. Kontsevich, 'Frobenius manifolds and formality of Lie algebras of polyvector fields', Int. Math. Res. Notices 4 (1998), 201-215.

[4] La. J. Barrott and Navid Nabijou, 'Tangent curves to degenerating hypersurfaces' (2020). URL: https://arxiv.org/abs/2007.05016.

[5] M. Blickle, 'Cartier isomorphism for toric varieties', J. Algebra 237(1) (2001), 342-357.

[6] K. Chan, S.-C. Lau and N. Conan Leung, 'SYZ mirror symmetry for toric Calabi-Yau manifolds', J. Differ. Geom. 90(2) (2012), 177-250.

[7] K. Chan, N. C. Leung and Z. N. Ma, 'Geometry of the Maurer-Cartan equation near degenerate Calabi-Yau varieties' (2015). URL: https://arxiv.org/abs/1902.11174.

[8] K. Chan and Z. N. Ma, 'Smoothing pairs over degenerate Calabi-Yau varieties' (2019). URL: https://arxiv.org/abs/ 1910.08256.

[9] T.-M. Chiang, A. Klemm, S.-T. Yau and Eric Zaslow, 'Local mirror symmetry: calculations and interpretations', Adv. Theor. Math. Phys. 3(3) (1999), 495-565.

[10] T. Coates, A. Corti, S. Galkin, V. Golyshev and A. Kasprzyk, 'Mirror symmetry and Fano manifolds', in European Congress of Mathematics (European Mathematical Society, Zürich, Switzerland, 2013), 285-300.

[11] A. Corti, M. Filip and A. Petracci, 'Mirror symmetry and smoothing Gorenstein toric affine 3-folds' (2020). URL: https://arxiv.org/abs/2006.16885.

[12] V. I. Danilov, 'The geometry of toric varieties', Uspekhi Mat. Nauk. 33(2) (1978), 85-134.

[13] P. Deligne and L. Illusie, 'Relèvements modulo $p^{2}$ et décomposition du complexe de de Rham', Invent. Math. 89(2) (1987), 247-270.

[14] B. Fantechi, M. Franciosi and R. Pardini, 'Deformations of semi-smooth varieties' (2020). URL: https://arxiv. org/abs/2010.02296.

[15] S. Felten, 'Log smooth deformation theory via Gerstenhaber algebras', Manuscr. Math. (2020), 35 pages. URL: https:// link.springer.com/article/10.1007/s00229-020-01255-6.

[16] D. Fiorenza, M. Manetti and E. Martinengo, 'Cosimplicial DGLAs in deformation theory', Comm. Algebra, 40(6) (2012), 2243-2260.

[17] R. Friedman, 'Global smoothings of varieties with normal crossings', Ann. Math. 118(1) (1983), 75-114.

[18] M. Gross, L. Katzarkov and H. Ruddat, 'Towards mirror symmetry for varieties of general type', Adv. Math. 308 (2017), $208-275$.

[19] M. Gross and B. Siebert, 'Mirror symmetry via logarithmic degeneration data. I', J. Differ. Geom. 72(2) (2006), 169-338.

[20] M. Gross and B. Siebert, 'Mirror symmetry via logarithmic degeneration data, II', J. Algebraic Geom. 19(4) (2010), 679-780.

[21] M. Gross and B. Siebert, 'From real affine geometry to complex geometry', Ann. Math. 174 (2011), 1301-1428.

[22] A. Grothendieck, 'Éléments de géométrie algébrique. I. Le langage des schémas', Publ. Math. Inst. Hautes Etudes Sci. 4 (1960), 5-228.

[23] A. Grothendieck, 'Techniques de construction en géométrie analytique. III. Produits fibrés d'espaces analytiques', Séminaire Henri Cartan 13(1) (1960-1961), 1-11.

[24] A. Grothendieck, 'Éléments de géométrie algébrique. IV. Étude locale des schémas et des morphismes de schémas. II', Publ. Math. Inst. Hautes Etudes Sci. 24(231) (1965), 5-231.

[25] A. Grothendieck, 'Éléments de géométrie algébrique. IV. Étude locale des schémas et des morphismes de schémas. III', Publ. Math. Inst. Hautes Etudes Sci. 28(255) (1966), 5-255.

[26] K. Hashimoto and T. Sano, 'Examples of non-Kähler Calabi-Yau 3-folds with arbitrarily large b2' (2019). URL: https://arxiv.org/abs/1902.01027.

[27] B. Hassett and S. J. Kovács, 'Reflexive pull-backs and base extension', J. Algebr. Geom. 13(2) (2004), $233-247$.

[28] D. Iacono and M. Manetti, 'An algebraic proof of Bogomolov-Tian-Todorov theorem', in Deformation Spaces (Vieweg + Teubner, Wiesbaden, Germany, 2010), 113-133.

[29] L. Illusie, 'Frobenius and Hodge degeneration. Introduction to Hodge theory', in SMF/AMS Texts and Monographs, 8 (American Mathematical Society, Providence, RI, 2002). Translated from the 1996 French original by James Lewis and Peters, 96-145.

[30] F. Kato, 'Log smooth deformation theory', Tohoku Math. J., Second Series, 48(3) (1996), 317-354.

[31] F. Kato, 'Log smooth deformation and moduli of log smooth curves', Int. J. Math. 11(02) (2000), $215-232$.

[32] K. Kato, 'Logarithmic structures of Fontaine-Illusie', in Algebraic Analysis, Geometry, and Number Theory (Baltimore, MD, 1988), (Johns Hopkins University Press, Baltimore, MD, 1989), 191-224.

[33] L. Katzarkov, M. Kontsevich and T. Pantev, 'Hodge theoretic aspects of mirror symmetry, in From Hodge Theory to Integrability and TQFT tt*-Geometry, Vol. 78 of Proc. Sympos. Pure Math (American Mathematical Society, Providence, RI, 2008), 87-174.

[34] Y. Kawamata and Y. Namikawa, 'Logarithmic deformations of normal crossing varieties and smoothing of degenerate Calabi-Yau varieties', Invent. Math. 118(3) (1994), 395-409.

[35] N.-H. Lee, 'd-Semistable Calabi-Yau threefolds of type III', Manuscr. Math. 161 (2020), 257-281.

[36] N.-H. Lee, 'An example of non-Kähler Calabi-Yau fourfold' (2021). URL: https://arxiv.org/abs/2102.12656.

[37] K. Liu, Y. Shen and X. Chen, Applications of the Affine Structures on the Teichmüller Spaces, Vol. 154 of Springer Proc. Math. Stat (Springer, [Tokyo], 2016). 
[38] C. Nakayama and A. Ogus, 'Relative rounding in toric and logarithmic geometry', Geom. Topol. 14(4) (2010), $2189-2241$.

[39] A. Ogus, Lectures on Logarithmic Algebraic Geometry, Vol. 178 of Cambridge Studies in Advanced Mathematics ( Cambridge University Press, Cambridge CB2 8BS, UK, 2018).

[40] M. C. Olsson, 'Universal log structures on semi-stable varieties', Tohoku Math. J. (2) 55(3) (2003), 397-438.

[41] H. Ruddat 'Log Hodge groups on a toric Calabi-Yau degeneration', in Mirror Symmetry and Tropical Geometry, No. 527 in Contemporary Mathematics (American Mathematical Society, Providence, RI, 2010), 113-164.

[42] H. Ruddat, 'Local uniqueness of approximations and finite determinacy of log morphisms' (2018). https://arxiv.org/abs/ 1812.02195.

[43] H. Ruddat and B. Siebert, 'Period integrals from wall structures via tropical cycles, canonical coordinates in mirror symmetry and analyticity of toric degenerations', Publ. Math. Inst. Hautes Études Sci. 132 (2020), 1-82.

[44] S. Schröer and B. Siebert, 'Toroidal crossings and logarithmic structures', Adv. Math. 202(1) (2006), $189-231$.

[45] J. H.M. Steenbrink, 'Mixed Hodge structure on the vanishing cohomology', in Real and Complex Singularities (Proc. Ninth Nordic Summer School/NAVF Sympos. Math., Oslo, 1976) (1977), 525-563.

[46] J. H.M. Steenbrink, 'Logarithmic embeddings of varieties with normal crossings and mixed Hodge structures', Math. Ann. 301(1) (1995), 105-118.

[47] The Stacks Project Authors, Stacks project. URL: http://stacks.math.columbia.edu/.

[48] T. Tsuji, ' $p$-Adic étale cohomology and crystalline cohomology in the semi-stable reduction case', Invent. Math. 137(2) (1999), 233-411.

[49] T. Tsuji, 'Poincaré duality for logarithmic crystalline cohomology', Compositio Math. 118(1) (1999), 11-41.

[50] T. Tsuji, 'Saturated morphisms of logarithmic schemes', Tunis. J. Math. 1(2) (2019), 185-220.

[51] N. Tziolas 'Smoothings of Fano varieties with normal crossing singularities', Proc. Edinb. Math. Soc. (2) 58(3) (2015), 787-806.

[52] N. Yotsutani, Global Smoothings of Degenerate K3 Surfaces with Triple Points (2020). URL: https://arxiv.org/ abs/2004.03162.

[53] N. Yotsutani, 'Notes on diffeomorphism classes of the doubling Calabi-Yau threefolds' (2021). URL: https://arxiv. org/abs/2101.11841. 